University of Louisville

ThinkIR: The University of Louisville's Institutional Repository

Electronic Theses and Dissertations

8-2016

\title{
Care, constraint, and collaboration : situating gender and power among multidisciplinary human service organizations.
}

Christa Jane Moore

University of Louisville

Follow this and additional works at: https://ir.library.louisville.edu/etd

Part of the Family, Life Course, and Society Commons

\section{Recommended Citation}

Moore, Christa Jane, "Care, constraint, and collaboration : situating gender and power among multidisciplinary human service organizations." (2016). Electronic Theses and Dissertations. Paper 2496. https://doi.org/10.18297/etd/2496

This Doctoral Dissertation is brought to you for free and open access by ThinkIR: The University of Louisville's Institutional Repository. It has been accepted for inclusion in Electronic Theses and Dissertations by an authorized administrator of ThinkIR: The University of Louisville's Institutional Repository. This title appears here courtesy of the author, who has retained all other copyrights. For more information, please contact thinkir@louisville.edu. 
CARE, CONSTRAINT, AND COLLABORATION:

SITUATING GENDER AND POWER AMONG MULTIDISCIPLINARY

HUMAN SERVICE ORGANIZATIONS

\author{
By \\ Christa Jane Moore \\ B.A., Western Kentucky University, 1998 \\ M.A., Western Kentucky University, 2005

\begin{abstract}
A Dissertation
Submitted to the Faculty of the

College of Arts and Sciences of the University of Louisville in Partial Fulfillment of the Requirements

for the Degree of
\end{abstract}

Doctor of Philosophy

In Applied Sociology

Department of Sociology

University of Louisville

Louisville, Kentucky

August 2016 
Copyright 2016 by Christa Jane Moore

All rights reserved 



\section{CARE, CONSTRAINT, AND COLLABORATION: SITUATING GENDER AND POWER AMONG MULTIDISCIPLINARY HUMAN SERVICE ORGANIZATIONS}

By

Christa Jane Moore

B.A., Western Kentucky University, 1998

M.A., Western Kentucky University, 2005

A Dissertation Approved on

August 5, 2016

by the following Dissertation Committee:

Patricia Gagné, Ph.D., Department of Sociology, Dissertation Committee Chair

David Roelfs, Ph.D., Department of Sociology, Committee Member

Robert M. Carini, Ph.D., Department of Sociology, Committee Member

Gul Aldikacti Marshall, Ph.D., Department of Sociology, Committee Member

Kristina Zierold, Ph.D., Department of Epidemiology and Population Health, Committee Member

Corrie Rice, Ph.D., EKU University Training Consortium, Committee Member 


\section{DEDICATION}

This dissertation is dedicated to my parents

Mr. Jerry D. Moore, Sr. and Mrs. Brenda Lou Moore,

for their unconditional love and constant encouragement,

to my ever-supportive life partner,

Mr. Ron Alexander Johnson,

to my loving and life-long companions, my siblings,

Mr. Jerry D. Moore, Jr. and Mrs. Natalie Lou Moore Marshall, and to my beloved family,

especially the dear ones we have loved and lost.

Your spirit lives on in my works

and will continue to inspire me. 


\section{ACKNOWLEDGEMENTS}

It is with an incredible sense of gratitude that I first want to acknowledge my mentor, faculty advisor, and phenomenal dissertation chair, Dr. Patricia Gagné. Without your constant guidance and enduring, unconditional support I would not have experienced such a tremendous sense of success in the Applied Sociology Doctoral Program. To my committee members, Dr. Dave Roelfs, Dr. Bob Carini, Dr. Gul Marshall, Dr. Kristina Zierold, and Dr. Corrie Rice, I offer deep appreciation for your interest, time, and availability, as well as your inspiring insights and incredible tenacity. Thank you for sticking with me all the way to the finish line.

I also want to acknowledge the faculty and staff of UofL's Department of Sociology. Thank you for supporting student success and for creating such engaging opportunities for personal and professional growth. To my extraordinary classmates, many of whom have become dear friends, I acknowledge all the caring ways you helped to pull me through the tough times. You celebrated every single accomplishment no matter how large or small.

To my WKU community, too, I am so appreciative of the professional opportunities you have extended to me as well as the many different ways you continue to support my academic journey. I am incredibly fortunate to have received the love, patience, generosity, and infinite support of my family and friends. You are my constant companions, and I could not have done all of this without each and every one of you. 


\begin{abstract}
CARE, CONSTRAINT, AND COLLABORATION:

SITUATING GENDER AND POWER AMONG MULTIDISCIPLINARY

HUMAN SERVICE ORGANIZATIONS
\end{abstract}

Christa Jane Moore

August 5, 2016

Child maltreatment has long been recognized as a pervasive social problem that affects a vast number of children and families each year (Finkelhor 1991; Krug, Mercy, Dahlberg, and Zwi 2002; Horwath and Morrison 2007; Hood 2014). An interdisciplinary team approach to family services has been commonly accepted as the best practice for intervening to protect children from maltreatment; however, prior research has identified significant gaps in establishing and maintaining collaborative relationships across multidisciplinary organizational domains. Interdisciplinary power structures and diverse philosophical orientations have been identified as barriers to effective collaborative exchange; thus, a more in-depth exploration of the influences of gender and power on interagency collaboration is needed (Bardach 1998; Langhout and Thomas 2010; Hurlburt, Aarons, Fettes, Willging, Gunderson, and Chaffin 2014).

This study incorporated a feminist approach to the use of multiple qualitative methods by conducting participant observation, focus group interviewing, and in-depth interviewing of women and men affiliated with a diverse representation of human service organizations. An applied research partnership with a multi-county human service 
organization provided entrée to the study population. Through analytic induction using a grounded theoretical approach the study explored perceptions of power, authority, gender, inequality, and bureaucratic constraints that emerged during organizational processes of interagency collaboration among multidisciplinary human service organizations (Charmaz 2014; Corbin and Strauss 2014). Findings indicate that establishing relationships is critical for interagency collaboration to be effective; however, the lived experience of interagency collaboration is that ethics of care and care work are constrained by gendered power dynamics, primarily ethics of justice embedded in bureaucratically-structured human service organizations situated within a plurality of complexities. Further, tensions between bureaucracy and ethics of care are enacted through relative, subjective, and exclusionary forms of gendered and other types of intersectionally-situated bureaucratic power. 
TABLE OF CONTENTS

ACKNOWLEDGEMENTS ........................................................................ iv

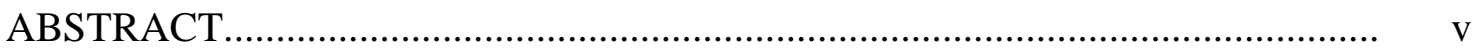

CHAPTER ONE: INTRODUCTION ........................................................ 1

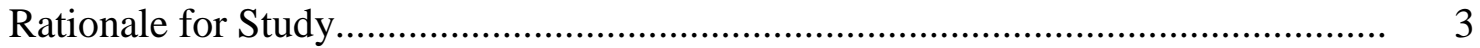

Child Maltreatment...................................................................... 5

Crisis Intervention Services and Interagency Collaboration....................... 10

Potential Contributions of the Study............................................................ 11

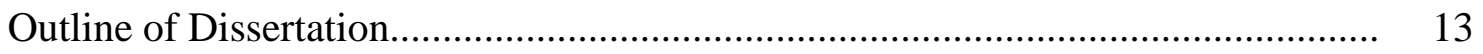

CHAPTER TWO: REVIEW OF LITERATURE............................................... 16

Human Service Organizations................................................................... 16

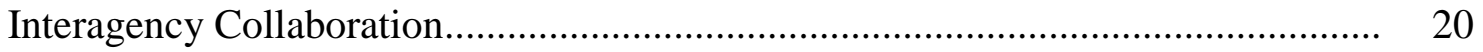

Defining Collaboration.................................................................. 22

Barriers to Collaboration....................................................................... 26

CHAPTER THREE: THEORETICAL FRAMEWORK..................................... 30

Organizational Perspectives on Power.............................................................. 31

Types and Characteristics of Power......................................................... 33

Sources of Power............................................................................... 37

Expressions of Power................................................................... 38

Feminist Perspectives on Power.............................................................. 44 


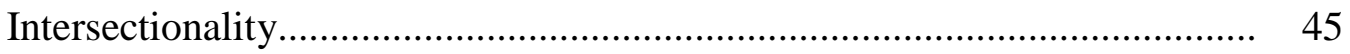

Standpoint Theory.................................................................... 47

Organizational Feminism and Power.......................................................... 47

Research Questions................................................................... 50

CHAPTER FOUR: METHODOLOGY ...................................................... 51

Rationale for Qualitative Methods.......................................................... 53

Sensitizing Concepts.................................................................. 54

Ethical Issues, Roles, and Relationships.............................................. 56

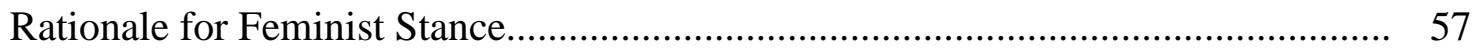

Feminist Research.................................................................... 58

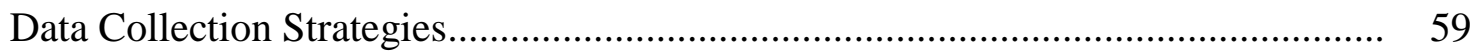

Gaining Entrée through the Host Agency............................................. 60

Description of the Sample Population................................................... 60

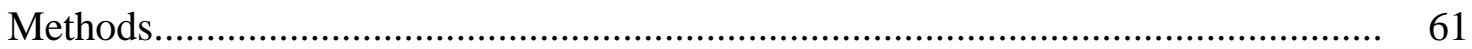

Participant Observation.................................................................. 62

Focus Group Interviewing................................................................ 65

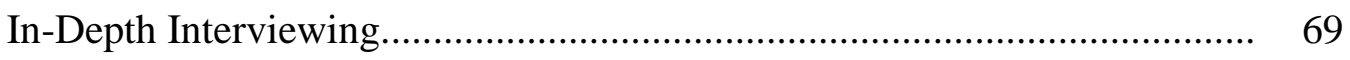

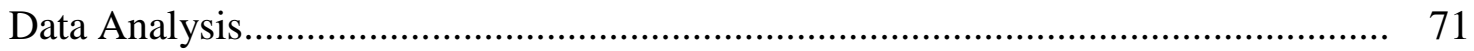

Validity and Reliability.............................................................. 72

CHAPTER FIVE: FINDINGS ................................................................

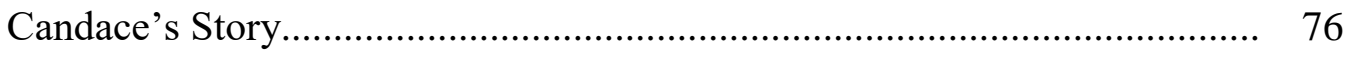

Contextualizing the Previous Study Findings....................................... 94 
Relationships and Interagency Collaboration............................................... 95

Establishing Relationships.............................................................. 99

Lived Realities of Interagency Collaboration............................................. 106

Gender and Other Institutional Intersections...................................... 107

Ethics of Care and Ethics of Justice.................................................. 115

Professionalism and Ethics.............................................................. 117

Additional Bureaucratic Constraints................................................ 127

Power and Interagency Collaboration........................................................ 132

Relative Power........................................................................... 133

Subjective Power.................................................................... 140

Exclusionary Power..................................................................... 142

CHAPTER SIX: DISCUSSION AND CONCLUSIONS ................................ 152

Gender, Power, and Collaboration 152

Intersectional Hyper-Complexity................................................... 157

Limitations of the Study.............................................................. 167

Implications for Future Research................................................. 169

Recommendations for Enhancing Interagency Collaboration................... 171

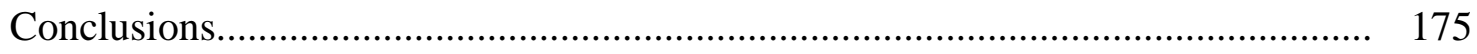

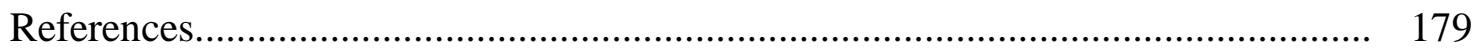

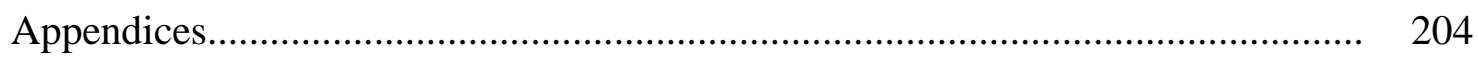

Curriculum Vitae................................................................................ 213 


\section{CHAPTER ONE INTRODUCTION}

The child welfare system is a densely populated organizational environment consisting of myriad human service agencies that work together to accomplish integrated, family-focused tasks (Horwath and Morrison 2007). Previous scholars have explored patterns of interagency cooperation, coordination, and collaboration and have consistently found both strengths and barriers stemming from collaborative exchange (Kagan 1993). Envisioning ways in which interagency collaboration can be enhanced so as to directly impact the lives of at-risk children and families is a complex and challenging task (Gray 1989; Kagan 1991). Barriers to collaboration are multilayered and thickly striated with ideological, organizational, and philosophical disparities. These disparities are steeped in power.

Although traditional ideologies about the constitution of "a normal family" may remain a barrier at a deeper, unconscious level of institutionalized inequality, an acknowledgement of the immeasurable ways in which increased collaboration can make a pronounced difference in families' lives may be a realistic starting place (Langhout and Thomas 2010; Kania and Kramer 2011). Acknowledging the need for better collaboration supports the foundational principle of creating agencies and programs focused on supporting families and children in need. An explicit goal in many cases is matching the appropriate agencies, programs, or services to specific family needs thereby reducing the degree of duplication in service provision (aiim.org 2015; Sowa 2008). 
The more human service organizations cooperate to serve families in the need, the greater the number of families and the greater the number of needs that will be met. Cooperation may be challenging though when competition or other contentions for power induce organizational resistance. Another goal of interagency collaboration, then, is to match the needs of families to specific human service organizations and program services so that the needs of families may be targeted and distributed among the agencies with whom they are involved and subsequent action steps can be formulated along with accountability measures. This "wrap-around" orientation to providing human services has gained popularity over the last decade and has become a best-practice model for confronting risk and safety issues associated with child maltreatment (Bruns, Walker, Bernstein, Daleiden, Pullman, and Chorpita 2014; NWIC 2016).

Agencies practicing more effective methods of communication and cooperation may experience enhanced interagency collaboration when focused on the needs of at-risk children and families, especially within the context of family crisis (aiim.org 2015). Graduating from an acknowledgement of the need to enhance collaboration to practicing enhanced collaboration involves the principle of providing proactive family service as opposed to reactive services. There are two overarching principles to consider. First, agencies have a responsibility to inform at-risk families of their services, but this responsibility extends further to informing other human service organizations as well. Doing so allows families to make decisions based on informed consent and it also allows other organizations to offer accurate information to client families about the accessibility of available community resources. Second, building supportive interagency partnerships neutralizes barriers resulting from competition or philosophical differences. Establishing 
collaborative relationships based on social networking to meet the needs of children and families also secures greater organizational stability within an ecology of complex, multidisciplinary human service systems.

While a wrap-around orientation to interagency collaboration is the dominant model for meeting the needs of at-risk families and children, few studies have focused on organizational barriers to effective collaboration that are rooted in discipline-specific goals and authority structures. To address this gap in the literature, the dissertation research explored the organizational experience of participating in interagency collaboration using a feminist approach to multiple-methods research. Although prior studies have examined strengths and barriers of collaborative ventures between organizations, insufficient attention has been given to the implications for bureaucratic processes on multidisciplinary, multi-systemic, family-based services within the context of child maltreatment (Horwath and Morrison 2007; Sowa 2008). The use of feminist methodologies further allows for an opportunity to contextualize inherent power relations within traditional bureaucratic structures and the subsequent impact of disproportionate power on organizational relationships and on human services to families (Bernard 2001; McCall 2005).

\section{RATIONALE FOR STUDY}

This study of interagency collaboration is needed because of the seriousness of child maltreatment as a predominant contemporary social problem and a public health issue (Finkelhor 1991). Each year approximately 6.6 million children are referred to state child protective services (CPS), and nearly 3.2 million children nationally receive a CPS investigation or alternative CPS response for reports of child maltreatment (U.S. 
Department of Health \& Human Services 2015; Childhelp 2016). In 2014, approximately 702,000 child victims experienced at least one substantiated or indicated incident of maltreatment nationwide. Tragically, an estimated 1,580 children died during the federal reporting period as a result of child maltreatment, including 23 deaths attributed to child maltreatment occurrences in Kentucky. Nearly 80 percent of child maltreatment fatalities involved at least one parent as the perpetrator. In addition to the personal and societal costs of child victimization, there are exorbitant financial costs associated with child maltreatment including the costs of foster care and other residential or ancillary services (Childhelp 2016). The financial impacts of child maltreatment have been estimated to range as high as $\$ 124$ billion dollars per year.

The costs of family violence, both in lives and in local, state, and federal funding, are not our only concerns. From a sociological perspective family violence is symptomatic of deeper structural constructions of power, privilege, and inequality (Strauss, Gelles, and Steinmetz 1980; Miller and Knudsen 2007; Wallace 2002). Societal reactions to family violence have been inconsistent and contingent upon historical norms, ideals, trends, and cultural shifts; however, an increasingly formalized, bureaucraticallystructured organizational response to family dysfunction and violence has progressively taken shape since the 1960s (Winton and Mara 2000). While necessary and beneficial in many regards, the bureaucratic response to confronting deviance within families has also been complicit in further institutionalizing various forms and sources of inequality, including inequalities that shape the potential for family violence and child maltreatment. 


\section{Child Maltreatment}

Since the 1960s, child maltreatment has consistently gained more societal attention and has become a focal area for social research (Horner 2008). Child maltreatment, which includes both child abuse and neglect, is defined by federal legislation as "Any recent act or failure to act on the part of a parent or caretaker, which results in death, serious physical or emotional harm, sexual abuse, or exploitation, or an act or failure to act which presents an imminent risk of serious harm.” (H.R. 867 1997). The vast literature on child maltreatment typically emphasizes one or more of its constituent types: physical abuse, sexual abuse, emotional or psychological abuse, or neglect. Child maltreatment is generally viewed as a multidimensional, complex social problem that requires multi-systemic, complex organizational responses (Winton and Mara 2000; Crossen-Tower 2002). Interagency involvement across institutional domains necessitates the capacity for effective communication, relationship building, and collaboration at local, state, and federal levels (Butler, Atkinson, Magnatta, and Hood 1995; Horwath and Morrison 2007; Langhout and Thomas 2010).

Child welfare in Kentucky. According to the Kentucky Kids Count Data Book (Kentucky Youth Advocates 2015), one in four Kentuckians are children, comprising approximately 23 percent of the state's population. Children under five years old represent 27 percent of the child population $(1,012,614$ ages $0-17)$, children ages five to 13 years old represent 50 percent of the child population, and the remaining 23 percent consists of youth ages 14 to 17 years old. The racial and ethnic distribution of Kentucky’s child population consists of 81.7 percent Caucasian children, 10.8 percent Black children, 5.6 percent Hispanic children, and approximately 1.9 percent classified in 
state data as Other race or ethnicity. More than two in every five children, approximately 42 percent, reside in a high-poverty area of Kentucky; thus children exposed to poverty stricken areas may lack sufficient access to necessary resources or to higher quality resources such as education, health care, and safe neighborhoods. For example, in 2014 nearly 50 percent of incoming kindergarteners were not prepared for school and approximately 48 percent of fourth graders were not proficient in reading. These children also experience a greater vulnerability to the consequences of higher rates of crime, violence, and unemployment, among other risk factors (Krug, Mercy, Dahlberg, and Zwi 2002).

The urgency for community-based prevention and interventions becomes more prevalent when comparing Kentucky's county and regional data to statewide trends and in comparing state data to national trends. My doctoral research was initiated in Warren County, the third most populated county in Kentucky and the largest county in terms of square mileage and population size within its ten-county designated service region (referred to as the Two Rivers Service Area by Kentucky's Cabinet for Health and Family Services, Department of Community Based Services (CHFS 2016) or as the Barren River Area Development District, "BRADD," according to Kentucky Revised Statutes (KRS 147A 2016)). County data shows a number of high risk indicators for families and children residing in Warren County (Kentucky Youth Advocates 2015). Warren County exhibits a higher rate of children placed in out-of-home care due to substantiated child maltreatment compared to the state as a whole, a rate of 53.3 children compared to 37.2 children per 1,000 ages 0-17. Warren County also has a higher rate of 
youth incarcerated in the juvenile justice system, a rate of 56 children compared to a state rate of 37.5 children per 1,000 ages 10-17.

A comparison of Kentucky's statewide child welfare data to national child welfare data shows similarly alarming trends (US Department of Health and Human Services 2013). Nationally, 3,016,794 child maltreatment referrals were made to state child protection agencies during federal fiscal year 2013 (September 2012 - October 2013). Of the 78,056 child maltreatment referrals made to Kentucky's child protective services (CPS) agency, 70.7 percent met the criteria for a formal CPS investigation or assessment compared to 60.9 percent nationally. The overall rate of referrals made per 1,000 children in the population was 77.0 for Kentucky while the rate nationwide was 47.1. Similarly, Kentucky had a higher rate of CPS investigations or assessments compared to national child welfare data, 69.9 versus 42.9 (per 1,000 children in the population), and a higher child victim rate, 19.7 compared to 9.1 nationally. Although there were no distinct differences in child gender, Kentucky's largest proportion of child victims tended to be younger than the age distribution reported nationally, 36.1 percent were ages birth to three years in Kentucky compared to 33.8 percent nationwide. Perhaps due to higher statewide indicators of need for families and children in Kentucky, there were also discrepancies in the availability of post-CPS response services compared to national trends. Nearly 90.7 percent of child victims in Kentucky received post-response services compared to only 63.8 percent of child victims reported nationally.

The pervasiveness and severity of child maltreatment concerns has necessitated the installation of broad scale, multidisciplinary, complex human service systems as a societal response to family and child protection. Within the scope of my previous 
internship study and the current doctoral research, two prominent human service organizations have served as nexus points wherein the complexities of systemic integration through interagency collaboration could be observed and studied: Child Advocacy Centers (CACs) and Family Preservation Programs (FPPs) (Moore 2014). CACs and FPPs in Kentucky. Children who have experienced victimization as a result of child maltreatment, and, in particular, sexual or severe physical abuse, are commonly referred to a regional CAC for Multidisciplinary Team (MDT) planning, forensic clinical interviews, and forensic medical examinations (National Children's Alliance 2016). CACs focus on serving child victims of sexual abuse and investigating the criminal nature of reported child sexual assaults; however, non-sexual physical assaults may also be a reason for referral (Walsh, Cross, Jones, Simone, and Kolko, 2007; National Children's Alliance, 2013). The number of CACs throughout the nation have increased dramatically, thus demonstrating the extent of sexual abuse as a serious social problem. As of 2007, the number of state and local CACs joining the National Children's Alliance had grown from only 22 in 1992 to more than 650 accredited or associated centers (Walsh et al., 2007).

CACs originated with the goals of ensuring greater physical and emotional safety for child victims of sexual abuse and as a means of increasing the likelihood of successful criminal prosecution of identified sexual perpetrators (National Children's Alliance, 2016). CACs have achieved these goals through the coordination of MDTs composed of multiple human service disciplines including medical professionals, trained forensic clinical interviewers, therapists and psychologists, attorneys and judges, police personnel, child protective services, and other key representatives from various social service 
organizations. The MDTs are engaged in following the CACs' focused model of collaboration, the Child Advocacy Center Model, which was developed to clarify, define, and guide the role each partner agency plays in collaborative service planning with and for client families (National Children's Alliance, 2016).

Another critical service provided to families with children at imminent risk of removal from the home due to safety concerns related to child abuse and neglect are Family Preservation Programs. Family Preservation Programs (FPPs) have an established history of providing in-home crisis intervention services to families with children at risk of removal due to child maltreatment (NFPN 2015). Legislatively mandated in most states including Kentucky, FPPs follow a research-based model and are one of the most rigorously structured family-centered interventions available across the nation. The impetus for FPPs is the goal of preventing children from being removed from their homes and from being placed in out-of-home-care settings, primarily state-paid foster care systems (CHFS, 2008).

Within Kentucky, this goal is also of fiscal primacy due to suppressed economic conditions and an increasingly high number of children entering state-paid placements including foster care, residential treatment facilities, and private childcare placements funded in lieu of a sufficient number of available state-operated foster homes (CHFS 2016). Removing children from their families of origin is traumatic and potentially detrimental to a child's wellbeing even if an incident of abuse or neglect has previously occurred in the home (Kinney, Happala, and Gruyter 1991). In-home crisis intervention programs have been found to be effective in engaging families in their treatment options, 
thereby challenging the issue of limited client rights to choice during service provision involving substantiated child maltreatment (Staudt and Drake 2002).

Much research has focused on child maltreatment, associated social issues, and the viability of different intervention options including CAC and FPP models (Berry 1997; Fraser and Haapala 1991; Fraser, Nelson, and Rivard 1997; Institute for Family Development 2015). As such, CACs and FPPs are considered to be model crisis intervention programs that promote both family involvement and multidisciplinary engagement through an intensive use of interagency collaborations (Kirk, Kim, and Griffith 2005; NFPN 2015).

\section{Crisis Intervention Services and Interagency Collaboration}

Societal responses to child maltreatment are multidisciplinary and include a wide array of human service organizations procedurally designed to "wrap around" a family during periods of crisis, dysfunction, or instability (Crosson-Tower 2002). Child protection services (CPS), law enforcement, and the judicial system are typically the first organizations to respond to alleged child maltreatment. Medical practitioners, mental health providers, family advocates, and other community resources, such as FPPs, may provide additional services (Sowa 2008). An interdisciplinary team approach to family services has been commonly accepted as best practice for intervening to protect children from first-time or recurrent child maltreatment as well as to provide treatment options for family healing and recovery (Smith, Witte, and Fricker-Elhai 2006).

Previous research has documented that human service organizations may be more open to partnering together through organizational processes such as interagency collaboration when families, particularly children, are determined to be in crisis and at 
risk of physical or emotional harm (Grady and Chen 2009). FPPs and similar crisisintervention programs are tasked with providing direct services to families whose children are at imminent risk of removal from the home for safety reasons. An important aspect of FPP services is the engagement of multi-disciplinary, inter-organizational collaborative partnerships in response to one or more incidents of substantiated child maltreatment (NFPN 2015).

FPP was selected as the host agency for the dissertation research for a number of important reasons. The FPP model of services requires the facilitation and recruitment of interagency collaboration across the human services organizational domain. Additionally, FPP's crisis orientation to intensive family needs often results in expedited inter-

organizational responses. My professional background and experiences include previous employment as a FPP worker, supervisor, and program director as well as a contract assistant for state-funded in-home service programs throughout the state of Kentucky; therefore, I have extensive institutional knowledge about the interworking of FPP as well as the external organizational environment.

\section{POTENTIAL CONTRIBUTIONS OF THE STUDY}

My research has the potential to significantly contribute to the existing literature on interagency collaboration within the human services organizational field in three important ways. First, prior studies have explored organizational factors that influence interagency collaboration, such as resource dependency and social exchange of costs and benefits (Bardach 1998; Sowa 2008). The current study adds a feminist orientation (explained more fully in Chapters Three and Four) that explored hierarchal power relationships among organizations, particularly those imbued with authority (Acker 
2008). Feminist research seeks to minimize the power dynamics between the researcher and the study participants and maximize opportunities for participant empowerment, especially if participants are constrained by circumstances of social oppression or inequality.

Second, the current study included a focus on systemic engagement and decisionmaking within an inter-organizational collaborative context. The quality, directionality, and extent of collaboration during decision-making were presumed to be associated with power and with the organizational structure of an interagency, interdisciplinary team (Bardach 1998). Third, this study made use of several qualitative methods oriented within a feminist research stance. The goals of these methods were to produce robust, informative findings from rich and specific data and engage in a more in-depth analysis of how gender and power shape the collaborative process (Charmaz 2014). Incorporating a feminist research stance also implies exploring opportunities to promote equity and social justice; therefore, the results of my study retained a focus on improving human services for at-risk families and children as well as for professionals who engage in care work (Gottfried 1996; Kleinman 2007).

Another major goal is to support professional practice in human services and to allow for a practical application of the findings in collaborative work settings, especially within the gendered institution of child welfare services. My findings have the potential to lead to many positive social changes including an increased understanding of power relations, more equitable organizational interactions, and, most importantly, better outcomes for victims of child maltreatment and their family members as well as the enhanced well-being of professional human service workers. 
The current study is an opportunity to focus on potential gaps in the interagency collaboration process. Few prior studies have explored the influence of differential organizational power and the influence of authority as potential gaps in multidisciplinary interagency collaboration, and this area of inquiry was also excluded in the previously completed doctoral internship; therefore, the current research explores the influence of power and authority on interagency collaboration from a feminist organizational perspective (Acker 2012; Moore 2014). I offer more than a feminist critique of organizational power and its impact on interagency collaboration. Indeed, a feminist stance also implies an obligation to identify possible alternatives to power-based social concerns (Gottfried 1996; Grosz 2013). Data-driven recommendations for enhancing interagency collaboration are proposed at the end of Chapter Six.

\section{OUTLINE OF DISSERTATION}

The dissertation consists of six chapters, including the present Chapter One, a reference section, supporting appendices, and my Curriculum Vitae. Chapter One presents an overview of interagency collaboration within the context of human services provided by organizations that target at-risk families and children as the primary service population. National, state, and local child welfare data were included as rationales for my study of interagency collaboration among multidisciplinary human service organizations.

Chapter Two provides a comprehensive review of the literature on interagency collaboration within the human services organizational context. Chapter Two is broken into three main sections, the attributes of human service organizations that differentiate them from other types of complex organizations, definitions and rationales for 
interagency collaboration, and descriptions of potential barriers. Within the section on how collaboration has been previously defined, the literature review focuses on how collaboration has been conceptualized as a feminine work mode and how rationales for collaboration illustrate gendered communication. Formalized definitions were also explored as demonstrated in policies, plans, and other forms of bureaucratic documentation. The second section, barriers to collaboration, emphasizes prior literature on organizational power and authority, challenges related to traditional, masculinized bureaucracy, and collaboration as a gendered process.

Chapter Three presents descriptions of the theoretical framework and constituent theoretical traditions that have explored the concepts of gender, power, and power relations. Organizational and feminist perspectives on power shape the primary framework culminating in their combined tradition, organizational feminism-a perspective with important implications for the dissertation findings.

Chapter Four describes my use of multiple qualitative methods and is divided into the following subsections: rationale for using the qualitative methodologies I employed; sensitizing concepts; ethical issues; rationale for my feminist stance and feminist research; data collection strategies; gaining entrée through the host agency; description of the sample population; methods used including participant observation, case file reviews, focus group interviews, and in-depth interviews; and data analysis strategies including validity and reliability.

In Chapter Five I present the analytic inductive findings. Although a previous survey was conducted during the doctoral internship and was used to inform the subsequent research activities, the dissertation findings focus on the qualitative research 
methodologies described in Chapter Four including participant observation, case file reviews, focus group interviewing, and in-depth interviewing. The previous survey was explicated in detail in the Doctoral Internship Report (Moore 2014) and during conference presentations presented in October 2015 and in February 2016 (Moore 2015; Moore 2016).

The last chapter, Chapter Six, highlights major findings of the research and discusses theoretical breakthroughs and applied implications for future studies, social policy, and collaborative practices; limitations of the study; recommendations for enhancing interagency collaboration; and, conclusions. Chapter Six is followed by a reference section, additional supplements contained in an Appendix section, and a copy of my Curriculum Vitae. The appendices include: (A.) the IRB-Approved Informed Consent Form; (B.) a blank copy of the Host Agency Agreement; (C.) the Focus Group Interview Guide; (D.) the In-Depth Interview Guide; and, (E.) a Human Services Acronym Guide. 


\section{CHAPTER TWO}

\section{REVIEW OF THE LITERATURE}

Child maltreatment is generally viewed as a multidimensional social problem that requires multi-systemic approaches provided across diverse organizational fields (Winton and Mara 2000; Rossen-Tower 2002). In Chapter Two, I review the previous literature on human service organizations structured within institutionalized systems of care and myriad organizational processes, primarily interagency collaboration, through which they converge.

\section{HUMAN SERVICE ORGANIZATIONS}

Human service organizations are structured within institutionalized systems of care (Mayhew 2012; Hasenfeld 2013; ACT 2016). Institutionalized systems of care, and, subsequently, human service organizations are integrated in complex ways designed to meet a complex range of individual and family needs throughout the life course. At a broader, cultural level, institutionalized systems of care function to stabilize society's most basic needs such as strengthening families, communities, education, healthcare, and justice. Thus, the human services field is comprised of a dynamically integrated spectrum of complex organizations with a vast complement of institutional missions, goals, and targeted outcomes.

Human service organizations differ from other types of formal and informal organizations (Hasenfeld 2013). First, people and human lives are the "raw materials" of human service organizations (p. 11). All organizations require one or more raw source 
materials through which a work process creates a product to be used, and, in capitalist societies, to be sold according to economic principles and consumer practices. "It is this transformation process to which people are subjected that defines them as the raw material of the organization, and it is precisely what differentiates human service organizations from other bureaucracies" (p. 11). Human service organizations, in addition to serving the needs of individuals and families, offer a structure for sorting, classifying, and categorizing the types of transformational processes to be used.

The moral intentions of human service organizations also set them apart from other types of organizations (Hasenfeld 2013; ACT 2016). The motives, actions, and interactions of human service workers are guided by moral intentions and principles just as the structure of human service organizations is guided by social judgments about individual behavior and individual worth. The institutional environments of human service organizations perpetuate and mirror broader cultural principles and install modes of legitimacy as anchors for their moral work including legislative and regulatory bodies, bureaucracies, professional associations and boards, and clients (Meyer and Rowan 1977; Hasenfeld 2010). Another key attribute of human service organizations is their pervasive and continuous efforts to manage indeterminacy (Meyers 1993; Sandfort 1999; Hasenfeld 2010). Human services are highly situational and contextual; therefore, widespread uncertainty and unpredictability require human service workers to become skillful professionals who are adept at using subjective discretion in their care work with potentially volatile clients (Hasenfeld 2010).

Human service workers have direct contact with and regularly develop relationships with their clientele, the individuals and families whose transformations 
become the focus of their work. The client-worker relationship, then, is another differentiating attribute of human service organizations (Cohen 1998; Altman 2008; Hasenfeld 2010). The client-worker relationship may be voluntary or involuntary, but it is the centrality of this relationship that often intensifies and complicates the indeterminacy of human services (Hasenfeld 2010).

The client-worker relationship is a personal, interpersonal, intersectional, and social relationship (Cohen 1998; Winton and Mara 2000; Altman 2008; Hasenfeld 2010). The quality of client-worker relationships becomes a critical aspect of human services when one or more of the following conditions exist: when clients are required to have continuous contact with human services; when a human service requires extensive biographical information, direct contact time, or access to the client's home or routine; when interpersonal communication is necessary for an intervention; when compliance is essential; and when the stakes are high, such as when concerns for an individual's safety are imminent (Hasenfeld 2010).

Within the context of this study, the attributes that human service organizations share with more traditionally-structured bureaucratic organizations are as important as the qualities that set them apart (Hasenfeld 2010). Feminist scholars have claimed that the underlying tensions situated within this contested space are gendered in orientation and result from the ways in which ethics of care conflict with legal-rational, bureaucraticallystructured ethics of justice (Acker et al. 1983; Gilligan 2000; Acker 2012; Noddings 2013). 


\section{Ethics of Care and Ethics of Justice.}

The modern history of human services originated in progressive philosophies and associated social movements of the early $19^{\text {th }}$ century (Crosson-Tower 2002). Growing from a religiously-fostered almsgiving tradition of primarily untrained volunteers, child maltreatment had received sporadic public attention up until the infamous case of Mary Ellen Wilson in 1874. Wilson, who was a child victim of extreme and recurrent abuse and neglect at the hands of her step-mother, was one of the first children to be officially removed from the custody of a parental guardian by a formal community organization, the Society for the Prevention of Cruelty to Animals (SPCA). The SPCA intervened because there was not yet a formal organization with a mission of child protection. The Society for the Prevention of Cruelty to Children (SPCC) formed a year later, and, on the heels of the notoriety of the much-publicized Wilson case, the social problem of child maltreatment gained broad-scale public support and cultural momentum. By the early 1960 's, child maltreatment became commonly viewed as a formal social problem and attenuated focus propelled federal, state, and local legislative and funding initiatives to systematize more complex organizational responses derived from traditions of care.

Human services evolved out of and were founded upon moral principles and professional ethics rooted within broader institutionalized systems of care (Hasenfeld 2000; O'Brien 2005; Clark 2006). Yet, the more bureaucratized human service organizations have become, the more these rationalized systems of bureaucraticallystructured service collide with mandates to care for families and children with crisis needs (Hood 2014; Hugman 2014). As conceptualized more fully in Chapter Three and explicated further in Chapter Five, the caring work of human services often collides with 
the bureaucratic dictates of what has become a highly rationalized system in ways that are, at their core, gendered, and often in complex and intersectional ways.

The cooperation and coordination of family-focused services through interagency collaboration is critical for the safety and well-being of at-risk children. Such a nexus of interagency involvement across institutional domains necessitates the capacity for effective communication, relationship building, and community partnerships (Butler, Atkinson, Magnatta, and Hood 1995; Horwath, Morrison 2007; Langhout, and Thomas 2010). It also requires an understanding of basic definitions and perceptions of collaboration as well as a deeper exploration of underlying hegemonic ideals that shape organizing practices and principles. However, as I demonstrate in Chapter Five, bureaucratic dictates, such as documenting every step, recommendation, program referral, and treatment plan, among other forms of organizational tasks, can consume the time, energies, focus, and resources of those whose work is, ostensibly, to care for and protect at-risk, neglected, and abused children and strengthen families.

\section{INTERAGENCY COLLABORATION}

There are many rationales for human service organizations to choose to integrate their work activities through interagency collaboration (Oliver 1990; Hudson 2004). Necessity may drive a collaborative venture as identified by a community or by a change in social policy or legislation. Reciprocity may emerge between organizations that share a field, mission, or other common goal — as in the case of child protection and supportive services to at-risk families. Sharing work and responsibility for meeting the needs of families and children may also seem more efficient. Organizational stability and legitimacy may also be fostered through the collaborative exchange of resources, in 
particular the distribution of clientele within a specialized service population such as atrisk families and victims of child maltreatment. It may also be the case that organizational asymmetry defines a collaborative landscape wherein some agencies disperse more power, authority, and influence thereby creating a coercive environment with few options outside of collaboration for financial autonomy and independence.

Collaboration within human services is necessary for comprehensively meeting the complex needs of at-risk families and children (Gray 1989; Kagan 1991; Kagan 1993; Bardach 1998; Sowa 2008). While this ideal is typically accepted as a basic social work principle, effective collaboration continues to be difficult to achieve and maintain (Sowa 2008; Harbert, Finnegan, and Tyler 2008). Barriers such as competitive funding for family programs, conflicting approaches to working with families, and institutional divisions among disciplines such as the medical system, the court system, the educational system, and governmental social welfare services greatly impact the ability of familyserving agencies to cooperatively work together (Harbert, Finnegan, and Tyler 2008). Beyond the prima facie barriers lie structural challenges shaped by systems of intersectional inequality (Collins 1998; Crenshaw 1991; McCall 2005). Structural gaps are harder to identify and confront, especially within an organizational context, because so many forms of intersectional inequality have shaped our everyday, taken-for-granted norms, values, beliefs, and behaviors. Organizations are commonly accepted as formal, rational systems through which we orient and frame routine social action; however, organizational behavior also reproduces and perpetuates institutional inequality based on gender, race, ethnicity, sexual orientation, nationality, and religiosity, among other sources of inequality (Perrow 2002; Perrow 2014). The power relations embedded within 
organizational interaction, including collaborative exchange, further influence how collaboration is defined and perceived and how barriers and gaps emerge. Increasing our awareness and understanding of the distribution of organizational power, though, is a feminist confrontation with the potential to significantly enrich interagency collaboration among human service organizations.

\section{Defining Collaboration}

Collaboration involves more than communication among agencies. It has been defined as "any joint activity by two or more agencies working together that is intended to increase public value by their working together rather than separately" (Bardach 1998). Collaboration is both a process for institutional cooperation and an arrangement between institutions (Gray 1989). Collaboration provides opportunities for different agencies to contribute varied perspectives of a problem or an issue. Other scholars propose that collaboration occurs on a continuum (Kagan 1991; Kagan 1993). For example, Kagan provided a four-part continuum that characterized the organizational relationships involved in the process of collaboration (Kagan 1991). Kagan's continuum included cooperation, coordination, collaboration (the act of), and service integration. Furthermore, collaboration may occur at various levels - policy, organizational, program, and client levels (Kagan 1993). This lack of consensus also contributes to the challenge of reciprocal and effective collaborative exchange (Sowa 2008).

Lack of consensus about how collaboration is defined and enacted may result from gender in addition to other personal characteristics or differences (Acker 2012; Barrett 2013). Collaboration has often been referred to as a "soft" set of skills, “emotional labor,” or "nurturant social skills" (Hochschild 1983; England 1992; Wharton 
1993; Kilbourne, England, and Baron 1994; Moss and Tilly 2004). In opposition to "hard" or traditional business skills associated with formal professionalism (e.g., educational attainment, physical strength, or specialized procedural knowledge, etc.), the collaborative skill set often includes an ability to interact with care, friendliness, enthusiasm, openness, willingness, and motivation to bridge philosophical or organizational divides. As a gendered organizational process, collaboration has been considered to be a feminine work mode.

Collaboration as a feminine work mode. Definitions and perceptions of collaboration are shaped by gender and gendered organizational processes (Coleman and Rippin, 2000). Organizational settings are traditionally hierarchical and often competitive (e.g., promotions, quota incentives, awards, etc.) within a masculinized orientation to work. Collaboration, as a feminine work mode, allows for more flexible definitions of cooperative ventures highlighted by greater mutuality, knowledge sharing, equity, trust and reciprocity.

Collaborative practices are counter to traditional, hierarchical, bureaucratic work modes in many important ways (McAllister and Dudau 2008). First, collaboration calls for sharing of work tasks and ideas about how work should be organized whereas traditional bureaucracy relies upon an ordered division of labor and restrictive, specialized functions subject to change upon managerial assignment. Second, collaboration may have a flexible leadership structure which conflicts with the tradition of a bureaucratic administrative hierarchy. Third, collaboration lends shared accountability to its team participants. In contrast, traditional organizations assume stricter managerial liability for operational ventures. 
Collaboration when perceived as a feminine work mode may further connote the idea of a "communal environment" based on shared commitments established through trust and a deeper, more informal degree of personal knowledge (Kanter 2008). This model deviates from more traditionally masculinized conceptions of bureaucracy wherein managers and subordinates conform to the professional veneer of impersonal, objective, task-oriented focus and commitment. The contrast between these two modes speaks to the distribution of power in an organizational setting. From a feminist perspective, this distinction is a key reason for promoting collaborative orientations to work, especially within human services to families and children.

Gender segregation in work tasks, work modes, and other organizational processes has undergirded much of what has come to be viewed as traditional and ideal about organizational norms (Reskin 1993). For example, social work and many other occupational roles in the child welfare system and the human services field continue to be dominated by women as representatives of a "caring" field much like nursing, teaching, and office assistance positions. A consistent problematic regardless of the occupation or role is the interface of traditional bureaucratic topography as the normative template for the cultural construction of work. The result is a masculinized orientation to organizational processes that makes collaborative exchange seem counterintuitive and difficult to communicate and define.

Defining collaboration through gendered communication. Collaboration is not only a gendered organizational activity; it is also rooted in gender-bounded communication (Murray 1996; McAllister and Dudau 2008). Definitions of collaboration are often contingent upon what McAllister and Dudau refer to as a "politics of presence," 
meaning a collaborative group's distribution of men and women and their unique disciplinary backgrounds, professional training, and organizational allegiances. As a socially constructed series of interpersonal relationships, the gendered social structure of collaborative teams directly influences how interagency collaboration procedurally operates.

Previous scholars have found that gender impacts perceptions about communication in the workplace (Coeling and Wilcox 1994). For example, in a study of collaborative exchange between male doctors and female nurses, researchers found that male physicians focused more on the content of communication and female nurses relied more upon relationship context. Additional studies have found differences in how men and women approach disagreements and conflict in collaborative environments (Allen 1997; Thomas, Sexton, and Helmrich 2003). These studies have consistently found that men tend to be more comfortable with expressing disagreements and handling overt conflict as part of collaborative ventures whereas women may be more focused on preserving interpersonal relationships.

Formalizing collaboration within human service. Definitions of interagency collaboration may also be formalized within organizational policies, procedures, contingency plans, and other sets of formal rules (Hudson, Hardy, Henwood, and Wistow 1999). Previous scholars have explored the formalization of collaboration as part of organizational, public, or social policy and have found some common rationales. First, collaboration has been viewed as a form of organizational altruism that conflicts with the bureaucratic structure and business goals of many agencies. Formalizing collaboration sets organizational boundaries and limits the degree of professional obligation as a 
rational procedure for demarcating paid work tasks. Second, collaboration without rules, procedures, or boundaries has been viewed as unpredictable, conflicting with the regularity and stability imposed through the "rhetoric of rationality" of a bureaucratic system. Such unpredictability exposes organizations to risks - personal, professional, and organizational liability - that could result in a loss of legitimacy, or even in legal actions, costs, and fees. Third, conceptions of collaboration may be higher-level administrative ideals that seem effective in theory, but that are unrealistic for front-line or direct human service workers to practically apply in a real-world situation (Lupton, North, and Khan 2001). Organizations rely upon bureaucratic norms, rules, and rationales; however, these organizing tools and processes also contribute to barriers to interagency collaboration.

\section{Barriers to Collaboration}

In addition to the varied, and often conflicting, definitions of collaboration, barriers may be tied to diverse organizational fields and differing institutional goals or philosophies (Darlington, Feeney, and Rixon 2005). For example, law enforcement and the judicial system have an obligation to uphold laws, investigate crimes, and prosecute criminal offenders. Meanwhile, CPS focuses on the protection of children and works to protect, strengthen, and preserve families at risk of crisis or dysfunction. Within human services, the organizational responses to individual and family needs are multi-faceted, dynamic, and complex; therefore, interagency collaboration, while ideal, can present myriad challenges.

Organizational power and authority. One area that has not yet been fully explored in previous studies is the distribution of power and authority in collaborative 
exchange among human services organizations. State-operated organizations, such as the courts and CPS, are tasked with immense responsibility for the safety, wellbeing, and permanency of children as well as the general welfare of individuals and families. Likewise, physicians and attorneys may yield more power, authority, and control over collaborative decisions as a result of their level of education and expertise. Such disparities in power, authority and control may strongly influence the accessibility, willingness, and quality of collaborative working relationships between human service agencies. An extensive exploration of types of organizational power and associated contexts will be presented in Chapter Three.

An extension of this concern must also consider the collaborative interaction between human service organizations and the client populations they serve. At-risk families do not just experience the stress of their interpersonal dynamics. Their condition may also be exacerbated by the unrealistic expectation that they are successfully able to access resources and effectively navigate the complex systems of community and governmental assistance programs on their own (Johnson and Cahn 2005). At-risk families may be discriminated against or viewed as more dysfunctional in their ability to manage their family lives when their difficulty maneuvering the complex array of services becomes apparent (Harbert, Finnegan, and Tyler 2008). Because there is typically no centralized point of intake or a shared information system for families across agencies, families are also required to recount their personal information and problems multiple times resulting in heightened strain, frustration, and service delays (Fitch 2009). This can add to the misrepresentation of who the family is and what they may be capable 
of were they to benefit from the support of more effective communication and collaboration among the multiple agencies involved in their lives.

At-risk families are disproportionately affected by poverty, lack of education, and lower socioeconomic status; therefore, they rely upon agencies to assist them with understanding information about their resource options and to help them with decision making (Johnson and Cahn 2005). If they receive conflicting information from different agencies that do not have collaborative working relationships with one another or with the family, then families become increasingly disempowered and constrained by their social circumstances.

Masculinized bureaucracy. Organizations are characterized by an ideal-type of hierarchal bureaucracy that is traditionally viewed as a masculine model (Britton 2000; Acker 2004). Within this framework gendered processes are inherently constrained by masculinized policies, practices, and interactions. Human service organizations are similarly structured, and, although the mission or goal (i.e., the product) of work is to help to strengthen families, a latent outcome of their masculinized bureaucracy is the reproduction of gendered and other forms of social inequality.

Previous studies have implicated masculinized bureaucracy as a barrier to effective interagency collaboration (Britton 2000). Traditionally, masculine forms of organizations rely upon a managerial hierarchy and a coercive implementation of formal rules, plans, and procedures. While such formalities are viewed as necessary for consistency, order, and organizational stability, they deter more participative forms of coordination, consensus-building, personal connectedness and relationships, professional empowerment, and sharing of power and leadership. Limitations in organizational 
flexibility and adaptation may drastically inhibit the motivation and ability of human service organizations to participate in interagency collaboration.

Collaboration as a gendered process. Interagency collaboration among human service organizations reflects the institutionalized norms, values, beliefs, processes, and other gendered structures of its constituent agencies; therefore, interagency collaboration, too, is a gendered process of intra-professional, inter-professional and interpersonal social interaction (Coleman and Rippin, 2000; Ely and Meyerson 2000). Gender is an "axis of power" within the organizational context and is both subtly and overtly rooted in the subtext of organizational narratives and micro-level processes (Coleman and Rippin 2000, p. 576).

Much research has shown that effective collaboration within a community of interagency partnerships may be the best method for enhancing the prevention of child maltreatment as well as decreasing intimate partner violence and other threats to family safety and wellbeing (Darlington, Feeney, and Rixon, 2005; Johson and Cahn 2005; Sowa 2008). Changing economic times, however, have not only affected the ability of families to maintain a stable household and employment but have also impacted access to federal, state, and local funding for family assistance programs. The confluence of greater family need for assistance and reduced accessibility to community resources because of local, state, and federal budget cuts demands even greater attention to opportunities for strengthening interagency collaboration (Johnson and Cahn 2005). 


\section{CHAPTER THREE}

\section{THEORETICAL FRAMEWORK}

My research is framed within two inter-related theoretical perspectives on power - organizational and feminist perspectives. Each theoretical perspective on its own lends robust explanatory potential. Blending organizational and feminist theories is necessary, though, because of the complexity of studying power relations at intersecting levels of structural, organizational, and interpersonal social interaction. Institutional inequality experienced through organizational processes affects each of us in our daily lives and is an "ideologically structured mode of action_-images, vocabularies, concepts, abstract terms of knowledge [that] are integral to the practice of power, of getting things done" (Smith 1987, pp. 1-2). Experiences are further shaped by personal biography; therefore, institutionalized inequality is a uniquely profound individual interaction with organizational processes and other social forces through which power is enacted.

Previous scholars have combined organizational and feminist perspectives on power to explore the many ways societal inequality has been reproduced within organizational settings as well as through organizational interactions (Acker 1992; Acker 2006). My study extends this literature by considering both the multidisciplinary nature of organizational collaboration among human service agencies and the differential power relationships between organizational types. While I relied on these theories to frame the focus of my research, I remained open to alternative explanations and used an analytic 
inductive approach to my data analysis with the goal of creating grounded theory as it emerged from the data.

\section{ORGANIZATIONAL PERSPECTIVES ON POWER}

Organizational perspectives explore the role and influence of organizations on social structure, social institutions, and social interaction (Perrow 1987). Organizations systematically influence much of contemporary society and they mediate human communication and social interaction to a large degree; thus, organizations exert power in our everyday taken-for-granted social lives. Prior studies of total institutions, those organizations that exert strict and rigid power, authority, and dominion over their members' daily lives including prisons, the military, and religious monasteries or convents, have demonstrated that "the essential core of organization is power" (Hardy and Clegg 2012, p. 756).

The multi-systemic nature of child welfare services requires an understanding of organizational and institutionalized power (Sowa 2008). Collaborative processes are complex and barriers to effective collaboration may be organizational or institutional in origin (Wood and Gray 1991). The complexity of collaboration may be associated with multiple organizational factors: stakeholder representation; autonomy; interactive processes; shared rules, norms, and structures; action or decisions; domain orientation; and desired outcomes. Environmental complexity, including organizational turbulence and uncertainty, may further shape collaborative ventures. Thus, both micro-level and macro-level processes interact with power relations.

Barriers may also result from opposing philosophical orientations of different organizations (Sowa 2008). For example, Johnson and Cahn (2005) noted the often 
conflicting roles and responsibilities of attorneys and child protection social workers. Attorneys, like child protection social workers, are burdened with proving a reported allegation of child maltreatment occurred; however, the philosophy of the court system has traditionally been punitive and justice-seeking while the role of the child protection agency has been restorative, seeking to serve the best interests of the child while preserving the family's intactness whenever possible. Court systems, including contemporary model courts such as family courts, are legislatively imbued with more power and authority than CPS or other human service organizations; therefore, disciplinary divides may be augmented by the legitimate exercise of power. Additional challenges to collaboration could be socio-political in origin and based on class divides, special interests, gendered relations and orientations, or other types of institutional divides through which power is disproportionately distributed (Johnson and Cahn 2005; Kennedy 2011).

Organizations influence and control much of everyday life (Perrow 2002). Max Weber (1978), as one of the earliest scholars to recognize the pervasive influence of bureaucratic power on culture, noted, "The power position of a fully developed bureaucracy is always great, under normal conditions overtowering (p. 991). The installation of extensive bureaucratic forms of social control has been deeply and ubiquitously embedded in the social construction of Western culture. Social constructions of culture rely upon social interaction and other participatory social acts; therefore, the control exerted by bureaucratic organizations does not appear to be coercive because it is tacitly accepted as normal, routine, and real. 
It is essential to understand the difference between, as well as the relationship between, power and authority (Weber 1947; Pfeffer 1981). Although questions about this relationship have been a subject of debate within the organizational literature, most scholars agree with the fundamental assertion that authority results from the legitimation of power. Power may be transformed into authority or "authoritative" positions may confer or be imbued with power; regardless, authority is an exercise of some type of power that is primarily expressed through institutionalized social structures, such as formal bureaucracies and organizations. The difference in power and authority is critical to an understanding of interagency collaboration because some human service organizations have (or are perceived as having) more legitimate authority than others. The disproportionate distribution of power and authority shapes the collaborative context for team decision-making about families and children at risk of abuse and neglect as well as the underlying definitions of what it means to be "at risk." An examination of different types and characteristics of power lends additional scope to the theoretical framework of my study.

\section{Types and Characteristics of Power}

The concept of power has been difficult for many scholars to operationalize because types and characteristics of power may be indirect, hidden, normative, or formalized (Dahl 1957). Power is situational as well as historically informed and, because it is inseparable from social interaction, all social institutions are imbued with various types and characteristics of power (Clegg, Courpasson, and Phillips 2006). A feminist stance on power recognizes that the everyday world is a problematic of power resulting from social constructions of a normative, or seemingly natural, social structure 
veiled within the masculine, thereby making power relations difficult to see or acknowledge (Smith 1987). Other scholars view power as a creative and empowering force with the potential to positively shape coordinated efforts both within and between organizations (Clegg, Courpasson, and Phillips 2006). Within the contexts of this research, different types, qualities, sources, and expressions of power are important to understand because interagency collaboration is contingent upon both positive and negative power dynamics.

Coercive and legitimate power. Prior research has identified multiple, and sometimes competing, typologies of power. Weber identified two primary types of power-coercive power and legitimate power (1941). Coercive power is the use of direct, indirect, or implied force to control behavior, in particular the behavior of subordinated individuals or groups, whereas legitimate power is perceived and commonly accepted as non-coercive authority. Legitimate power can be exercised through three types of authority: charismatic, traditional, and legal-rational.

Power and authority are interpretive relationships that occur within specific situational contexts (Clegg, Courpasson, and Phillips 2006). In organizational interactions, the structure of hierarchical administrative dominance is interpreted as legitimate power through which bureaucratic authority is distributed and exercised. Power is not one sided. Whether institutionalized or interpersonal power is dialectic and is the consequence of both submission and dominance. According to Simmel, habituation to the precepts of organizational life contributes to its acceptance as a normal, everyday routine (Simmel 1971; Clegg, Courpasson, and Phillips 2006). 
Subsequent scholars worked to flesh out Weber's typology further by adding that power can be exercised through forceful domination and manipulation (Goldhamer and Shils 1939). Moreover, power can be characterized as instrumental or non-instrumental, unilateral or bilateral, direct or indirect, upward or downward, and may be exerted in substitute forms or as sanctions. Power may be situational and can also take the form of influence (Foucault 1980; Mintz and Schwartz 1985; Handy 1993). Power is visible in its effects, thus regulated and organized social action often conforms to the influence of idealized and institutionalized representations of organizational life (Foucault 1977; Clegg, Courpasson, and Phillips 2006).

Additional typologies of power. The literature on power is immense and many scholars have constructed, expanded upon, or redefined similar typologies of power (French and Raven 1959; Lipkin 2013). Legitimate power within a stricter bureaucratic context refers to the hierarchal or managerial structure of administrative power, meaning a person in a higher position has decision-making authority and maintains control over others who fill subordinate or lower positions. Coercive power may be present when there are perceived or direct threats or force, but may also be exerted through organizational policies, procedures, contingency or strategic action plans, and other types of textual, documented bureaucratic rules that have a powerful coercive tendency.

Power may further be exerted as expert or informational types (French and Raven 1959; Lipkin 2013). Expert power is associated with the perception of those who are educated or who fill a specialized niche or organizational domain thereby possessing superior skills and knowledge. Closely associated with expert power is informational power, such as when an individual or an organization controls access to necessary or 
beneficial information. Reward power motivates or controls social behavior through an incentive-based reward structure such as raises, promotions, and acknowledgement awards. Connection power, closely associated with the organizational concept of social networks, refers to an association with a recognized person or organization who is perceived as being powerful. Referent power refers to an individual or organization's ability to inspire personal acceptance and approval through charismatic action, integrity, or other uniquely positive characteristics.

Many types of power are characterized by categorical inclusion and exclusion (Tilly 1998). Inclusionary and exclusionary boundaries may result in what is referred to as bounded pairs, a form of categorical power that leads to durable inequality. Referred to as binaries of power in feminist theory, bounded pairs include binary categories such as male/female, white/black, married/unmarried, heterosexual/homosexual, religious/nonreligious, and citizen/immigrant, to name a few. Categorical power is exerted through assumptions and stereotypes about both in-group and out-group members as well as individuals who are marginalized outside of bounded peripheries.

Power can also be intimate and tied directly to interpersonal relationships (Rudman and Glick 2012). A feminist stance on power reveals how emotional entrenchment contextualizes power in uniquely personal ways, ways that are influenced by and that interact with power at organizational and structural levels. Organizations consist of people who engage in a variety of interpersonal relationships; therefore, intimacy is an unavoidable factor that shapes the distribution of power and the implications of power. 
Hegemonic power. Power is hegemonic and deeply rooted in cultural ideologies, norms, traditions, practices, and beliefs (Lukes 1974; Doorewaard and Brouns 2003; Reed 2012). As one of the multiple "faces of power," a hegemonic conceptualization of power shifts it outside of direct human agency so that power relations become structural and institutionalized (Reed 2012, p. 30). For the purposes of this study of interagency collaboration among human service organizations, "The concept of hegemony expresses the casualness with which many people in various circumstances in daily life wield power or are subjected to it, without fully being aware of this form of influence" (Hamilton 1986, p. 3). Thus, hegemonic regimes and organizational systems of ideology distort and obscure true relations of power (Grosz 2013).

Hegemonic power within human services may take many different forms from traditional conceptions of legitimate family forms to isomorphic systems of organizational operations (DiMaggio and Powell 1983). The purpose of the current study is to explore how multiple forms of power, including hegemonic power, shape the landscape of interagency collaboration as well as to determine how power both emboldens and constrains collaborative ventures.

\section{Sources of Power}

Power has multiple sources (Pfeffer 1981). Power may be related to one's position within an organization, the degree of task specialization and expertise required to do certain types of work, or one's ability to shape and influence attitudes towards work as well as the actual work of others. A very important source of organizational power is dependence, having control of something another individual or organization wants or needs. Dependence becomes increasingly critical as a source of power when resources 
are scarce or limited. Power may also derive from uncertainty. Coping with uncertainty can be critical for organizational survival, especially because organizations rely upon bureaucratic rationality and predictability for stability. Organizations or services that are perceived as necessary and irreplaceable, such as the courts and CPS, are also viewed as powerful; therefore, monopolizing an organizational niche or domain may also be a source of power.

Expressions of Power

Sensemaking. Interagency collaboration has been viewed as a process of organizational sensemaking about the problems and needs of at-risk families and the human services best suited to help them (Head 2009; Wood, Büscher, and Ramirez 2012). Sensemaking through collaboration has been referred to as "an essential element of group culture” (Head 2009). Further, “...in sharing different perspectives, individuals in a group can create collective knowledge that leads to a common or shared sense of the task at hand" (p. 52). The creation of shared knowledge across human service organizations is imperative for making sense of families' crisis needs so that strategies of multi-disciplinary action can be formulated and enacted.

Three key aspects of sensemaking informed the study (Weick, Sutcliffe, and Obstfeld 2005). First, sensemaking as a function of interagency collaboration takes place through organizational categorization and communication which gives rise to shared understanding about "at-risk families," a bounded category that will be discussed further in the next section. Second, the exchange of interagency collaboration is an organizing process expressed in written contracts, case notes, formal recommendations, and other forms of documentation. Third, it is through the media of textual and verbal acts of 
interagency collaboration that the "invisible hand of institutions shapes conduct," in this case behavioral outcomes for families and children.

An essential element of sensemaking is categorization (Day and Lord 1992;

Weick, Sutcliffe, and Obstfeld 2005). As an organizing process, creating categories is a rational and efficient way to streamline information and tasks and make them meaningful; however, categories also explicitly and implicitly create power structures and shape power relations (Weber 1968; Tilly 1998). Categories are habitually bounded, paired, asymmetrical, and institutionalized.

Within the organizational domain of human services, categorizing types of families, their problems, and their needs is a form of institutionalized sensemaking that imposes differential power relations. Referring to a family as an "at-risk family" implies a deviation from other bounded categories of families such as a "healthy family," “functional family," "good family," or "traditional family." Creating typologies of child maltreatment — sexual abuse, physical abuse, emotional abuse, and neglect — do not only impose bounded categories, but also identifies deviations from legitimate and acceptable parenting practices. Sensemaking within human services is necessary to ensure the safety and wellbeing of children and other family members; however, a latent function is asymmetrical power relations based on resulting stigma and inequality.

Inequality becomes durable inequality when bounded categories, especially bounded pairs, are institutionalized (Tilly 1998). Also referred to as "social closure" the sensemaking process of creating categories that are open to one group but closed to another group results in disempowerment, oppression, exploitation, and opportunity hoarding (Weber 1968, p. 43). An example of social closure that is relevant for this study 
is the formalization of professional culture relative to the exclusion of recipient families

(Hugman 2014). According to Sáez and Sánchez (2006),

Moreover, exclusion has a relational nature: somebody is excluded (marginalized, left out) in comparison to someone else, who is located inside a particular social order or network. Exclusion is intrinsic to human and social life. It has always existed, no matter how visible or invisible it has been. The knowledge that societies have about exclusion only transforms the extent and nature of social exclusion producing new practices and ways of excluding. Social professions, as we currently see them, came into existence and developed as a result of new contexts of exclusion that required new approaches and methods of intervention, mostly funded and guided by states and social policies. (p. 600)

Institutionalized inequality may contribute to the formalization of

disempowerment as part of the bureaucratic sensemaking process of creating policies, procedures, rules and plans, and, in particular, statutes and other forms of state and federal legislation (Mikkelsen 2012). Child maltreatment is not only an illegitimate and deviant parental act it is also a crime; however, deeper issues of hegemonic power impregnate formal bureaucracy. For example, labeling child maltreatment as a form of deviant parenting subject to criminal prosecution is a relatively recent cultural shift in societal responses (Pfohl 1977).

It was not until the early 1960s that child maltreatment was broadly "discovered" to be a pervasive social problem (Pfohl 1977). Up until this time and throughout most of human history, the institution of the family was considered to be a private domain, a separate sphere, within which parents were deemed to be the best judge of how to raise and discipline their own children (Coontz 1992). Harsh, even torturous and unusual forms of child discipline were commonly accepted as a cultural norm based on the religious edict "spare the rod, spoil the child" as well as deeply held cultural beliefs about the sovereignty of family life and privacy in the home (Coontz 1997). 
The cultural shift in the United States was prompted by the release in 1962 of a major medical publication that identified the "child battering syndrome" as a serious medical concern and, ultimately, a prevailing social problem (Kempe, Silverman, Steele, and Silver 1962). Recognition and acceptance of child battering syndrome as a viable and legitimate social concern was largely based on the power of medical expertise; thus, beliefs about parental rights and family sanctity relative to the vulnerability and rights of children began to change. On the heels of what is now referred to as the Kempe Report strategic acts of federal legislation installed a governmental presence in monitoring parenting practices and the potential for child maltreatment including the Federal Child Abuse Prevention and Treatment Act of 1974, the Adoption Reform Act of 1978, Title XX of the Social Security Act which included the Social Services Block Grant and Aid to Families with Dependent Children, the Adoption Assistance and Child Welfare Act of 1980, the Child Abuse Victim's Rights Act of 1986, and the Victims of Child Abuse Act of 1991 (Sagatun and Edwards 1995).

Categorization, codification, and, eventually criminalization of parenting behavior came to be adopted by states who were required to integrate federal legislation into their local statutes. Changing laws, as forms of legitimate power and authority, influenced (and were mutually influenced by) the progression of changing hegemonic ideals that further shaped the corporatization of a societal response to child maltreatment through the creation of such organizational domains as the child welfare system and the human services field. Child welfare today, having further evolved through continuing acts of formalized sensemaking, heavily relies upon the integrated services of multidisciplinary 
human service organizations, many of which operate programs that are mandated by state or federal laws, such as CACs and FPPs in Kentucky (KRS 194A.050, KRS.620).

In addition to the installation of a large-scale bureaucratic child welfare system tasked with confronting the pervasive social problem of child maltreatment, other cultural trends resulting from organizational sensemaking have emerged. Cultural imperatives now, in comparison with prevailing ideals prior to the 1960 s, dictate a communal responsibility to protect children from identifiable and preventable sources of harm. Furthermore, childhood has come to be viewed as a sacred period of development and children are considered to be naturally innocent, vulnerable, and susceptible to harm, and in need of consistent supervision and protection (Aries 1962). Correspondingly, there is a culture of parental scrutiny and distrust bolstered by a system-of-care orientation to externally resolving family problems within a community of formalized organizational resources. These cultural shifts in the societal response to potential maltreatment has resulted in tremendous attention to the safety and wellbeing of children, and has displaced the sanctity of the private family domain - especially if a family is perceived as being outside of acceptable bounded categories. Therefore, sensemaking can be a form of categorical power that perpetuates durable institutional inequality (Tilly 1998).

Resource dependence. As a major theory within the organizational tradition, resource dependence has been broadly adapted by previous scholars. Within the context of my dissertation research, resource dependence is a foundation for understanding interagency collaboration among human service organizations (Gray 1989; March and Simon 1993; Bardach 1998; Pfeffer and Salancik 2003). As a quid pro quo model of collaborative exchange, resource dependence implies an obligatory stance on behalf of 
organizations that work together often and share a field, niche, or other specialized domain or target population. For human service organizations, resource dependence may speak to deeper motivations, especially for private or non-profit agencies or small programs that rely upon collaborative exchange as a means of ecological survival. From this perspective the collaborative act of parsing out responsibility for the targeted needs of at-risk families and children not only installs a broader, multidisciplinary approach, but it also produces work, the basis of organizational stability, as well as interdependence. Being part of a collaborative, multidisciplinary team approach further secures organizational legitimacy, a principal resource for autonomy, recognition, and, ultimately, power.

The importance of resource dependence and organizational interdependence among human service organizations is further illustrated by Bronfenbrenner's Ecological Systems Theory (1979). This ecological model has been broadly used to show the different forms of intersectional interaction that occurs between family members and their larger environment or community. Ecological Systems Theory also demonstrates the interdependence between organizations that work together to serve families and children as consumer clients. Supporters of this theory claim that a breakdown between the family and their environment of organizational supports can lead to increased stress and dysfunction resulting in the potential for child maltreatment. The same may be true for resource dependent organizations vying for organizational stability.

Organizational interdependence is based on several factors that support the formation of cooperative ventures such as interagency collaboration among human service agencies (March and Simon 1993). First, sharing a mutual dependence on one or 
more limited resources may promote organizational interdependence. For human service organizations, the pool of local at-risk families and children can be unstable and unpredictable because child maltreatment is most often an unplanned and impulsive occurrence. Contract funding and other financial resources may also fluctuate resulting in further resource scarcity.

Second, interdependence may be commensalistic or symbiotic (Gray 1989). From an ecological context, a commensalistic relationship between two or more organizations means that at least one of them benefits from the existence of another; however, it does not affect the organization it benefits from in either a positive or a parasitic manner. A symbiotic relationship in comparison describes an interdependent or cooperative environment wherein organizations are integrated and linked. Human service organizations may be both commensalistic and symbiotic because they are connected through mutually dependent resource transactions in the form of service integration. The mutuality of their organizational interdependence is a foundation for the integrated structure of collaborative social networks.

\section{FEMINIST PERSPECTIVES ON POWER}

Feminist theories provide a lens through which differential power relations within and across human service organizations, such as the child welfare system, can be observed and better understood (Stark and Flitcraft 1988). Two feminist perspectives, intersectionality and standpoint theory, are considered in further framing the general approach to the dissertation research, the methods used, and the subsequent data analysis. 


\section{Intersectionality}

Intersectionality theory refers to the multilayered and complex lives of human beings wherein multiple social systems, institutions, and social power structures intersect resulting in increased vulnerability to prejudice and discrimination (Crenshaw 1991; Collins 1998; McCall 2005). The intersectional nature of such social characteristics as race, gender, sexuality, and social class compounds experiences of inequality and discrimination. Intersectionality recognizes that privilege and discrimination result from the intersection of multiple identities and also acknowledges the interaction between privilege and discrimination that works to bolster and perpetuate both (Symington 2004). While feminist theory holds that social institutions are gendered, the use of intersectionality in this research has the potential to expose how organizations that purport to be collaborative may also work to reproduce racial, class, and two-parent hetero-centric inequality. An intersectional analysis may also expose how power is distributed and exercised during interagency collaboration among human service organizations, especially within the context of services to families or children who are marginalized through a matrix of compounded sources of institutionalized inequality (Symington 2004). Thus, this study has the potential to expand upon organizational theory with a feminist examination of the multiple pathways through which power can intersect in organizational, institutional, and interpersonal ways.

Further, an intersectional analysis allows us to recognize that a majority of at-risk families are poor and uneducated (Ajar and Benjet 1994; Kennedy 2011). Many are minority families as well as single-parent (primarily single-mother) families. Poverty, race, and class issues further intersect with the social stigma of alleged drug and alcohol 
abuse; criminal history; domestic violence; homelessness and transiency; physical and mental disabilities; and serial relationships or indiscrete promiscuity—risk factors that can be difficult for social service agencies to disregard or resolve (Finkelhor 1991; Schumacher, Slep, and Heyman 2001). Often the assumption alone of any one of these risk factors is sufficient to meet the requirements that a child may be at risk of suspected or alleged child maltreatment and to initiate a child protection investigation.

The challenges that struggling parents and children face are layered within a governmental system that privileges heterosexual, two-parent married families and that seeks out such a traditional family structure as the "ideal" institutional definition of family (Kennedy 2011). The intersection of poverty, race, class, and non-traditional (i.e., deviant) parenting behavior may magnify perceived risks to child safety; but, more importantly for this study, is the consideration of intersections of authoritative and nonauthoritative organizations. Families within the social service system experience significant social stigma and are often deemed untrustworthy and dishonest; therefore, their resource needs may be minimized, disregarded, or ignored. Although each family's case is deemed as unique and is supposed to be treated as such, there may exist a culture of faceless reproach within human services to the extent that poor, minority, lgbt, and/or single-parent families are seen as being less than ideal in their family make-up and possibly more in need of community resources and support. Such beliefs may lead to attitudes and behaviors that translate into potential gaps in effective collaboration among family-serving agencies. 


\section{Standpoint Theory}

My study acknowledges that human services organizations are made up of individuals with their own biographical backgrounds, personal experiences, and families; therefore, the qualitative aspect of this research embraces a standpoint theory perspective (Smith 1987). Feminist approaches seek to understand and validate the personal experiences of the research participants. The participants are viewed as more than subjects of a study. They are seen as individuals with shared or similar social experiences that are also situated within organizational and cultural systems of power and authority. The researcher does not seek to dominate the participant, nor does she seek to interpret the participants' experiences through her own sociological perspective; rather, the feminist researcher looks for ways to represent the social experiences of the participants from their own points of view. Traditional modes of sociological research may objectify the research subjects and treat them as separate, external from the research process and inferior to the 'skilled scientist' or the 'professional inquirer.' Adding standpoint theory to an intersectional organizational theoretical perspective may reveal the effects of differential power and authority on both organizational workers as well as the families they serve.

\section{ORGANIZATIONAL FEMINISM AND POWER}

The social institution of the family as well as the organizations that provide human services to families are gendered institutions (Acker 1992). According to Acker,

“The term 'gendered institutions' means that gender is present in the processes, practices, images and ideologies, and distributions of power in the various sectors of social life" (1992, p. 567). The intersections of these institutional processes as situated within the 
dynamics of at-risk family functioning and child maltreatment present opportunities for intervention rife with gendered politics. For example, previous studies have shown that the gender of judges, attorneys, and plaintiffs has influenced bias towards women during court experiences as well as decision making and family outcomes (Martin, Reynolds, and Keith 2002)

Much like interagency collaboration between human services organizations, inequality is complex and multidimensional. The underlying mechanisms of this 'multidimensional complexity' require further exploration, particularly when considering group-based inequality compounded further by poverty and other forms of socioeconomic inequality. Systemic inequality is not only personal; it is interpersonal and permeates the everyday lived experience of culture through the reproduction of its social institutions (Ridgeway 2013).

I use organizational feminism to explore the complexity of interagency collaboration among agencies that exert disproportionate power and authority during decision-making with and about at-risk families and child victims of maltreatment. My research is an effort to increase our understanding of the multidimensional complexity of inter-organizational interaction within the broader organizational domain of human services and the child welfare field. The application of organizational feminism in combination with intersectionality and standpoint theories may lead to significant breakthroughs in an understanding of how power shapes and is enacted through interagency collaboration. 


\section{Gender and Power}

Gender is taken for granted in everyday life (Connell 2002). Often viewed as a fixed personal and social characteristic of identity, binary gender distinctions are predominantly taken-for-granted as natural and normal. Efforts to sustain binary gender distinctions sustain cultural beliefs about gender and perpetuate power relations that stem from gender categories. Broader categories of inequality, such as income inequality or political inequality are often situated within gender inequality. Gender, then, is a basis for social interaction and shapes what individuals, groups, and even organizations do.

As a social structure, social constructions of culture, communication, and meaningful social activity (e.g., work within organizations) are patterned within conceptualizations of gender (Connell, 2002). Institutionalized patterns of gender arrangements may be referred to as "gender regimes" and are considered to be a normal feature of organizations and organizational interaction (p. 53). Previous scholars have suggested that gendered inequality involves at least four dimensions of gender relations: power relations, production relations, emotional relations, and symbolic relations. All of these forms are situated in history, culture, discourse, and social interaction and shape the relationship between gender and power.

\section{Gendered Substructures and Subtexts}

Gendered substructures make it possible for assumptions about the biological, intellectual, professional, and cultural differences between men and women to be embedded and reproduced within organizational processes (Acker 2012). Substructures such as wage inequality, job descriptions and workplace designs, behavioral policies, and 
the allocation of managerial promotions and subsequent decision-making power are entrenched in the normal, rational, hierarchal routines of bureaucratic entities.

Differential power is also implicitly exercised through gendered subtexts (Acker 2012, p. 217). Subtexts exist as an extension of gendered substructures and refer specifically to the ways gender is fixed in text, discourse, and common organizational

practices (e.g., evaluations). Gendered subtexts are implicit in everyday organizational interaction and, although they seem normal and natural, they supplant the equitable distribution of power between men and women.

\section{RESEARCH QUESTIONS}

My research seeks to answer three predominant research questions:

1. What is the role of power in influencing interagency collaboration among human service organizations that target at-risk families and children?

2. What forms of power shape the social interactions and outcomes of team-decision making in interagency collaborations?

3. What role does gender play in shaping power and the structure of interagency collaboration among human service organizations? 


\section{CHAPTER FOUR}

\section{METHODOLOGY}

I came into the doctoral program as a career professional in child welfare and human services; however, strategies for how to enhance the process of interagency collaboration eluded me. My experiences of coordinating integrated services with other professionals as a working clinician informed me that I was not alone in feeling this way. The doctoral dissertation presented opportunities to explore the influence of power on the gendered and intersectional processes of interagency collaboration among human service organizations. My doctoral research took place in multiple organizational settings and involved thorough reviews of child welfare data and reports, case file reviews, participant observation, focus group interviews, and in-depth interviews in addition to informal interviews and discussions with over 200 individuals involved in human services across 24 organizations and 35 distinct programs (see Table 1 for a summary of the research activities).

Throughout the one-year timeline of the study, May 15, 2015 - June 30, 2016, I witnessed many forms of interagency collaboration. In addition, I engaged in numerous discussions of how it was perceived, defined, enacted, and constrained according to the experiences of a diverse representation of participants. The findings submitted in Chapter Five explore their candid accounts and rich descriptions. 
Table 1. Characteristics of the Doctoral Research Activities

\begin{tabular}{|l|c|c|r|r|}
\hline $\begin{array}{l}\text { Research } \\
\text { Activities }\end{array}$ & Location & Dates & $\begin{array}{c}\text { Number } \\
\text { of } \\
\text { Sessions }\end{array}$ & $\begin{array}{c}\text { Number } \\
\text { of } \\
\text { Hours }\end{array}$ \\
\hline \hline $\begin{array}{l}\text { Participant } \\
\text { Observation }\end{array}$ & $\begin{array}{c}\text { Family } \\
\text { Preservation } \\
\text { Program }\end{array}$ & $05 / 01 / 2015-05 / 30 / 2016$ & 178.5 & 126 \\
\hline $\begin{array}{l}\text { Case } \\
\text { Reviews }\end{array}$ & $\begin{array}{c}\text { Family } \\
\text { Preservation } \\
\text { Program }\end{array}$ & $05 / 01 / 2015-05 / 30 / 2016$ & 45 & 50 \\
\hline $\begin{array}{l}\text { Focus Group } \\
\text { Interviews }\end{array}$ & $\begin{array}{c}\text { Family } \\
\text { Preservation } \\
\text { Program }\end{array}$ & $07 / 30 / 2015,11 / 06 / 2015$ & 2 & 4.25 \\
\hline $\begin{array}{l}\text { In-Depth } \\
\text { Interviews }\end{array}$ & Multiple & $08 / 18 / 2015-04 / 30 / 2016$ & 40 & 63.48 \\
\hline \hline \multicolumn{4}{|l|}{ Total Hours } & 243.73 \\
\hline
\end{tabular}

The process of interacting with human service professionals throughout the doctoral research richly informed my understanding of interagency collaboration, but also my understanding of organizational environments. I did not anticipate the degree of open access I was permitted nor the depth of individual experiences that were shared, and I sifted through hours of audio recordings and pages of transcriptions, field notes, child welfare data, reports and agency texts to discover a multiplicity of interpretive meanings.

The dissertation research used multiple qualitative methods. In-depth interviews were the primary qualitative method selected for exploring interagency collaboration among human service organizations (Padgett 1998; Singleton and Straits 2009). Prior to conducting in-depth interviews additional qualitative methods were used including participant observation, client case file reviews, and focus group interviewing. Qualitative methods offer rich description thereby surpassing some of the major 
limitations and criticisms inherent of quantitative methods (Singleton and Straits 2009). Qualitative methods have also been found to be useful in research with human service organizations (Padgett 2008; Sowa 2008).

Prior social research on families has consistently demonstrated the immensely complex nature of family dynamics and related organizational responses within the child welfare system (Finkelhor 1991; Strauss, Gelles, and Steinmetz 2006; Finkelhor 2009). Using a qualitative approach allowed for an understanding of the study of organizational responses to at-risk families particularly when families and children become susceptible to intimate abuse and violence. The broad scope of this study further required the use of qualitative methods in an effort to better understand the experiences of interagency collaboration among a local subset of family-serving organizations. The goal was to gain a better understanding of motivations, strengths, and potential gaps within collaborative relationships, especially gaps related to differential power and authority exerted during collaborative decision making and planning.

\section{RATIONALE FOR QUALITATIVE METHODS}

The use of qualitative methods allows the researcher to access a more holistic view of the sociological area of focus according to the perceptions and the lived experiences of the participants (Miles and Huberman 1994; Saldaña 2014). For the purposes of this dissertation, qualitative methods are best suited to developing understandings of processes, such as the exercise of power in groups (Charmaz 2014). Qualitative methods are also used to "bridge" research subjects into the research process and make them active participants in the research process, an important component of a feminist approach to doing qualitative research (Acker, Barry, and Essevold 1996). 
Involving participants in the research process is a critical opportunity to establish rapport, trust, and ongoing communication; therefore, using qualitative methods, such as qualitative interviews, is a more collaborative mode of conducting social science research.

Qualitative methods may be used to foster a feminist approach to social science research (Gottfried 1996). While quantitative, and some qualitative, research traditions advocate for maintaining distance and objectivity between the researcher and research subjects, a feminist approach to using qualitative methods urges a more interpersonal mode of research activity (Fonow and Cook 1991; Fonow and Cook 2005). Establishing rapport and trust on a personal, humanistic basis creates opportunities for open communication and more honest disclosure, especially about sensitive or emotional topics such as family dysfunction and child maltreatment. Approaching my research from a feminist stance on qualitative methods was an opportunity to access the lived experiences, or the standpoints, of the individuals involved in interagency collaboration so that a deeper, more personal understanding of their perceptions can enrich our understanding of structural barriers embedded in organizational processes.

Building upon the prior literature in this area, multiple qualitative methods were used to explore the perceived gaps of interagency collaboration related to power relations within a localized human services organizational environment as well as definitions, obligations, limitations, and motivations for collaborative exchange. Sensitizing Concepts.

Within the genre of qualitative research, and, in particular, an analytic inductive approach to grounded theory, the use of sensitizing concepts is necessary to determine a 
starting place and to interpret key qualitative findings (Bowen 2008). "Sensitizing concepts draw attention to important features of social interaction and provide guidelines for research in specific settings" (p. 14). Sensitizing concepts are indirect, often lacking "precise reference" and "clean-cut identification" (Blumer 1954). Sensitizing concepts inform the formulation, foundation, and direction of a qualitative study and promote theoretical sensitivity in the evolving theoretical framework for qualitative research.

Several important sensitizing concepts informed the dissertation research: power, inequality, and gender. Power as a sensitizing concept for a study of interagency collaboration among human service organizations was a critical assumption because of the complex intersections of structural, organization, and interpersonal levels of social interaction. The focal emphasis concerned the institutionalization of power and its pervasive hegemonic influence within and between these intersections. As a result of intersectional power distribution, the sensitizing concept of institutionalized inequality was presumed to occur, and one potential source of institutionalized inequality is gender, a third sensitizing concept. My orientation to sensitizing concepts was guided by the works of Charmaz (2003) who recommends using a constant comparison method during analytic induction and while using a grounded theory approach to conducting qualitative research. The use of a constant comparison method involves coding qualitative data and then constantly comparing the coded data to subsequent data as it is collected, analyzed, and categorized. Going back and forth between coded data and data yet to be coded allows a qualitative research to constantly compare sensitizing concepts to emerging categories and potential themes. My use of a constant comparative method and sensitizing concepts shaped and influenced the qualitative interview questions in addition 
to progressive revisions as new or refined sensitizing concepts and key findings emerged from the data.

Ethical Issues, Roles, and Relationships.

The ethical responsibilities associated with conducting social science research, and, in particular, qualitative research remained a primary concern throughout the study (Patton 2015). Qualitative research traditionally engages closer, more personal, research relationships between the social scientists engaged in a study and the participants. It was essential to keep in mind these important considerations:

1. Integrity — refers to integrity of the method as well as integrity of research agreements such as confidentiality, anonymity, and informed consent;

2. Flexibility — refers to the need to meet participants 'where they live,' both literally and figuratively by ensuring participants feel safe to be open and honest in a secure and private location as well as assessing the potential need to re-order or restructure the interview questions based on their series of answers.

3. Sensitivity — refers to the subject matter and any related sensitive issues or concerns.

4. Empathy—refers to having a sincere humanized conversation as part of the interview process with sincerity and true interest in participants' responses.

5. Follow up and follow through — refers to follow up on unclear statements and taking the time to interpret the interview responses through the participants' intent and meaning rather than making research assumptions. It is also vital to follow through on agreements made with individual participants during the data collection process. 


\section{RATIONALE FOR FEMINIST STANCE}

I approached this study from a feminist stance on organizational interaction. Organizations are gendered institutions wherein systems of hegemonic power and privilege are embedded in the bureaucratic process (Acker 2012). What this means for human service agencies that focus on the needs of at-risk families and children is that even though they operate within a traditionally-feminized system of care, they function within a cultural business model created by and for men. Framed within a mode of operation that gives primacy to masculinized bureaucracy, systems of care are preempted by systems of power and authority. Some of the consequences of this disproportionate integration may be -

1. Following homogenized policies, procedures, contingencies, and other organizational plans become prioritized work activities that compete with rather than inform or reinforce human services work provided to at-risk families in need;

2. Human service agencies who have minimal or sporadic contact with at-risk families, such as law enforcement, the court system, and, often CPS, exert greater organizational power, authority, and privilege compared to other, family-centered human service providers who provide more intensive services. Their extensive knowledge of at-risk families, in addition to their more personal interactions, inform their recommendations and their involvement in multidisciplinary collaborations; however, a perceived lack of authority or formal expertise may nullify or diminish their more informed recommendations made on behalf of disempowered families. 
3. At-risk families and children are transformed from individuals with unique cultural identities, needs, problems, and fears into business commoditiestargeted service goals that are a required condition of contractual funding, and, ultimately, continued organizational survival for many human service agencies.

\section{Feminist Research.}

Processes of knowledge creation have traditionally been male dominated; therefore, feminist research has several important principles. (Acker, Barry, and Essevold 1983; Smith 1987; Gottfried 1996). First, feminist research seeks to liberate traditional, exclusionary practices of positivistic social science so that women can engage in research activities and apply the results. Second, feminist research often engages equitable and participatory practices in an attempt to extricate social science from oppressive methods. Third, the practice of feminist research challenges dominant intellectual traditions and seeks to constantly reflect upon its own development.

Standpoint methodology. Conducting research presents another instance of how organizational power is exercised, legitimized, and institutionalized (Smith 1987; Harding 2004). Adopting a feminist stance to qualitative research, however, is an acknowledgement of the power relations inherent in the research process and the potential for social oppression. My research incorporated a standpoint methodology as a process of bridging my relationships with the study participants.

Interpreting and witnessing. Previous scholars have referred to qualitative methods, including in-depth interviewing, as an art of interpretation (Denzin 1994; Corbin and Strauss 2014). Humans communicate through storytelling and there are innumerable modes of contextual interpretation. One such mode of qualitative 
interpretation and inquiry is witnessing, a process of theoretical "scaffolding" that further integrates the shared roles of researcher and participants in co-constructing knowledge through narrative story-telling (Ropers-Huilman 1999, p. 21). Interpreting and witnessing have been used in prior feminist research to shape the representativeness and authenticity of data analysis and interpretation in ways that respect the unique perspectives of the study participants.

Witnessing, as a feminist stance to qualitative traditions of interpretation, refers to the researcher's obligation to honor the integrity of each participant's unique standpoint (Ropers-Huilman 1999). Another obligation, though, is to be accountable for presenting and interpreting findings based on participants' personal narratives to multiple audiences in a consistent way. In previous research witnessing has been used to study interagency collaboration and several additional research obligations were recommended as part of the practice of conducting qualitative research. Research is an active form of meaningmaking, but the researcher is only partially responsible for interpretation. As a witness, it is vital to represent meaning from the standpoint of each participant. Another obligation is to recognize that in conducting research there is the potential to change people, both participants and future audience members. We, as researchers, may also change, and we have an obligation to share that aspect of the research as well. Lastly, there is an obligation to share multiple experiences and meanings of power, gender, inequality, and other sensitizing concepts that shape our understanding of social reality and social justice.

\section{DATA COLLECTION STRATEGIES}

For the purposes of the dissertation research, I worked with a regional Family Preservation Program (FPP) to identify regularly participating collaborative partners who 
I then contacted and recruited for interviews. Having professional experience with providing FPP services at an earlier point in my human services career allowed me to understand the organizational terrain and boundaries within the situational context of FPP. A dissertation research agreement was negotiated with a regional FPP and I attained their support for this study (see Appendix B).

\section{Gaining Entrée through the Host Agency}

Entrée to the sample population was gained through a research collaboration with a multi-county Family Preservation Program (FPP), a family-centered human service offered through a local agency in Kentucky. FPP was selected as a viable gateway agency because of their mission and model which includes an obligation to seek out interagency collaborations with myriad other human service organizations in order to stabilize crisis-affected families and to prevent the reoccurrence of child maltreatment (Whittaker 1990; Fraser, Pecora, and Haapala 1991; Berry 1997; CHFS 2008).

\section{Description of the Sample Population.}

The sample population consisted of key informants who were agency representatives from human service organizations in Kentucky who regularly collaborated with representatives from a localized FPP. The organizations that participated were representative of a broad cross-section of professional concentrations including child protective services; law enforcement; the judicial system including courts and attorneys; mental health providers; the medical community; victim advocacy staff; and a selection of care workers from specialized human service organizations. An essential criterion for the sample population was the regular, and preferably recent (within the calendar year preceding the timeline for the study), collaborative involvement 
with FPP. While I tried to involve at least one woman and one man from each

organizational affiliation type, for many organizations only one participant was available to be a key informant.

Key informants are cultural insiders who inhabit social worlds and who tacitly understand the situational context for the taken-for-granted everyday lived experiences that occur within those settings (Shibutani 1955; Berg and Lune 2012). They have firsthand information and are knowledgeable about the meanings of situational behavior that a researcher as an outsider may not understand. Key informants from collaborating partner agencies were recommended by FPP staff as well as by other key informants according to a process of convenience sampling known as chain or referral sampling or snowball sampling (Biernacki and Waldorf 1981; Bergen 1993; Penrod, Preston, Cain, and Starks 2003). By using a process of chain referrals, I was able to obtain a convenience sample of highly integrated multidisciplinary organizational representatives who had consistent and verifiable experience with interagency collaboration. IRB approval was given for this study, and confidentiality of the participants was protected at all times.

\section{METHODS}

Qualitative methods were selected as the best methods for attaining rich, descriptive data about the collaborative experiences of key respondents from each of the different organizational affiliations, or disciplines. The use of qualitative methods often provides a theoretical depth and richness of clarification that is difficult to attain from survey data or other quantitative methods. 
Four qualitative data collection strategies were employed during the dissertation research — focus group interviewing, participant observation, case file reviews, and indepth interviewing (Berg and Lune 2012). The use of multiple qualitative methods, commonly considered to be part of a process of triangulation, has been recommended by previous social scientists as a means of convergent validation (Berg and Lune 2012). Convergent validation is an important consideration because a major criticism of qualitative methods includes poor reliability and validity related to the localized specificity of many qualitative studies. The use of multiple qualitative methods helped to counter the imposed perspective a single method may place on the social reality of a particular social setting or sample population.

The use of focus group interviewing, participant observation, case file reviews, and in-depth interviewing presented the best qualitative research options for my study because of the highly complex and specialized nature of human services as an organizational environment. Each method contributed specific strengths and limitations; therefore, a combination of these methods as part of a "multiple-lines-of-action" strategy reinforced the "depth of understanding an investigation can yield" (Berg and Lune 2012, p. 8). Combining these particular methods further bolstered my feminist approach to using qualitative methods because of the participatory nature each one offered to interactive research with participants who had opportunities to voice their unique concerns and perspectives. Giving voice to the participants influenced and strengthened the data collection, analysis, and interpretation as well as increased the authenticity and credibility of the findings.

\section{Participant Observation.}


Participant observation is a qualitative research method whereby the researcher becomes a participant of a social world in an effort to learn more about a particular social setting (Padgett 2008). Participant observers may be known or unknown to the people who interact within the social setting of interest, or they be fully active in the setting as opposed to passively observing interactions such as in the participatory action research context (Gottfried 1996). Used in combination with qualitative interviewing, participant observation allowed me to observe not only what people said about interagency collaboration, but also what they did. There are numerous roles one can take in participant observation to full member observer to observer and I adopted a primary role as a participant observer. (Adler and Adler 1987)

Over the thirteen months during which the doctoral research took place, one human service organization was used as a gateway agency through which entrée to the sample population was attained. In the following sub-section, I provide contextual information as a rationale for the selection of a regional FPP as the gateway agency and as a research setting for the qualitative methods used.

The Family Preservation Program. Participant observation within the regional CAC informed the creation of the Interagency Collaboration Survey (ICS) and the previous survey findings further contextualized the dissertation research, including the selection of a second host agency, a regional Family Preservation Program (Moore 2014). The questions prompted by the previous study led to an expanded research design adding qualitative methods intended to further explore the process of interagency collaboration among multidisciplinary human service organizations. I completed approximately 178.5 
hours of participant observation during which time I witnessed diverse opportunities for interagency collaboration including:

- staff meetings and case consultation

- staff workroom interactions

- file maintenance and case file review procedures

- supervisory meetings

- corporate administration visits and meetings

- home visits

- partner meetings and office visits

- communication modes and strategies (telephone, emails, texts)

- reporting and outcome tracking

- hiring and training of new and existing staff.

Case file reviews. As part of my participant observations, I was granted access to client files. I randomly sampled 45 FPP closed and completed client case files, meaning each client family formally accepted services and participated in the program through the point of service completion. Of the random sample, 100 percent were working with at least three other organizations in addition to FPP during the program intervention period; however, on average families served were involved with at least six other organizations in addition to FPP services. Some FPP families, approximately 23 percent of the random sample, were involved with as many as ten or more human service organizations concurrently with intensive FPP services. Participant observation findings as well as the findings of the randomized sample of case file reviews prompted the need for focus group interviews and subsequent in-depth interviews.

FPP serves an average of 3,050 clients families each fiscal year (a state fiscal year begins each July 1 and ends on the following June 30) (CHFS RFP.736 2012). By providing an array of specialized and intensive program services, FPP is able to directly support CPS' efforts to protect children considered to be at imminent risk of harm, and 
maintain the majority of them with their family of origin (NFPN 2016). In order to acclimate to the organizational setting, sensitize myself to regular and ongoing interagency collaborations, and attain a picture of the types of problems referred families experience that led to program referrals, I was authorized to pull and review client families' active and closed case files.

A research sample of 45 closed and completed case files was randomly selected for use in the dissertation. The sampling frame for the pool of case files for the random sample included four selection criteria:

1. The case was opened and served to successful completion, or "closed and completed," within the last three fiscal years.

2. The case received a minimum of four weeks of FPP in-home services.

3. The case file had been formally reviewed and closed out by an administrator; therefore, the case record of services had been effectively documented.

Completing the case file reviews was a valuable part of conducting participant observation because the descriptive service information allowed me to gain a greater context for the interactions I witnessed between FPP workers and professional partners as well as their service interactions with client families. The descriptions of community partnerships and integrated multidisciplinary services documented in the case files further provided another source of validation for the organizations discussed during the focus group interviews and for the organizational representatives I approached for participation in in-depth interviews.

Focus Group Interviewing.

Focus group interviews are a qualitative research method typically used with small groups in an effort to understand the shared experiences of group members (Berg 2011). A primary goal of focus group interviews is to moderate and facilitate a dynamic group discussion that stimulates the sharing of ideas, topics, problems, and solutions 
related to the social context of the group's purpose. Focus group interviewing is a strategic method for identifying group concerns or other particular focus of interest to the participants in a group, such as a group composed of front-line FPP caseworkers. When paired with participant observation, focus group interviews can be a powerful opportunity to observe group member interaction. The use of focus groups also presented an opportunity to verify or validate participant observations, and served as another source of constant comparative data.

One focus group was originally planned with the FPP staff. The week prior to the scheduled date of the focus group, the staff were provided with information about the goals and intentions of the focus group interview by the FPP supervisor who also clarified that staff participation would be voluntary, not mandatory. Six of eleven program staff were available to attend the first focus group during the day and time of their regularly scheduled weekly case consultation meeting. A second focus group was scheduled as a means of involving the remaining eleven staff members who voiced an interest and willingness to voluntarily participate in the focus group interview.

The goals of the two focus group interviews I conducted were to attain a list of multi-disciplinary agencies and representatives who regularly collaborate with FPP frontline staff and to learn about collaboration experiences from the FPP staff. An important aspect of focus group interviewing as a form of grounded theoretical work is to include information, concerns, and issues raised by the participants. Thus, conducting focus group interviews revealed an institutionalized understanding of the power dynamics in place between FPP and other human service organizations with which they share clientele, a critical resource for organizational survival. The focus group interviews 
directly shaped the areas and specific issues of focus I witnessed during participant observations and during in-depth interviews based on the information provided by the participants. Their insights were used to focus and refine participation observation activities and the in-depth interview questions.

Two focus group interviews were held with FPP employees during the time allocated for regular staff meetings. The first focus group interview took place in July 2015 and the second, a follow-up focus group interview, occurred in November 2015. The combined focus group interview time was approximately 4.25 hours. The first focus group interview was held with a mixed gender group of three men and three women. One participant was African American and the other participants were Caucasian. The second focus group interview included five FPP participants-one man and four women. The racial composition of the second focus group was mixed and consisted of participants who identified as multi-racial, African American, and Caucasian. Both focus group interviews were held during regularly scheduled weekly staff meeting times. Ages of the focus group participants ranged from early twenties to mid-sixties. Educational backgrounds included bachelor-level degrees in psychology, social work, family and consumer sciences, sociology, and divinity. All staff had been cross-trained in providing multiple types of crisis intervention services. FPP supervisors opted not to be present during the focus group interviews in order to avoid inhibiting open discussion among the program staff.

Several logistical aspects of the focus group interviews are also important to mention in relation to the findings. The focus group interviews were held on-site in the agency's conference room. The locations, days, and times for both focus group 
interviews were selected for the ease and convenience of the staff who regularly attended a weekly staff meeting according a pre-determined schedule. The staff were given prior notification by email and at previous staff meetings about the schedule for the focus groups and the focus of the doctoral study on interagency collaboration. Staff were previously informed by an organizational administrator of the voluntary nature of their participation in the focus group interviews. One staff opted not to participate in the first focus group because she would be on leave during the week it was scheduled to be held. I also explained the voluntary nature of focus group participation before beginning the focus group interviews. In addition, I reviewed and explained informed consent and provided hard copy IRB-approved Informed Consent forms for further review and signatures before beginning the interviews. I provided food and drinks as incentives for participation during both focus group interviews.

The conference room used provided sufficient space for all participants to sit comfortably around the same rectangular conference table. The room was the size of a moderate office space with sufficient sound barriers and a door to ensure privacy. I placed a sign on the door during both focus interviews that stated, "Meeting in Progress. Please Knock Before Entering.” All focus group interview participants gave consent for audio recording using primary and backup digital recorders.

I witnessed both focus group interviews to be dynamic and engaging. Multiple social and organizational factors seemed to shape the rapport and comradery of the two groups interviewed. First, the staff members had established interpersonal relationships and identified themselves as a cohesive working team of service providers. Second, the culture of the agency and of their program, FPP, adopted both formal and informal 
supportive contexts of a team mentality, including team meetings, team building, and team consultation. Third, the consensus of the two focus groups demonstrated solidarity and the validity of their responses. Peers prompted each other for clarification of similar types of collaborative experiences and denoted agreement through verbal affirmations and through gestures such as nodding or shaking their heads.

I used few planned focus group interview questions and few responsive prompts because of the limited time available for each focus group session, a timeframe of up to 1.50 hours. (You can cite Morgan on this, if you wish) Six primary focus group interview questions were asked (see Appendix $\mathrm{C}$ for the full questionnaire and focus group agenda):

1. Tell me about your experiences with collaboration?

2. Are there some agencies that are easier to collaborate with than others?

3. Tell me about your collaborations with specific agencies (a list was provided).

4. How would you rank the agencies listed in order of most decision-making influence to least decision-making influence?

5. Has your work been more successful as a result of collaboration?

6. What other information about interagency collaboration would be important for me to know?

Five of the six participants in the first focus group interview participated with open and interactive responses. One worker, a woman who was new and in her early twenties, was more quiet and unsure of how to contribute to the discussion. All five participants in the second focus group fully participated throughout the interview. Both focus group interviews profoundly enhanced my understanding of interagency collaboration among the human service organizations that partner with FPP, and they provided firm grounding for beginning and for continuing in-depth interviews. In-Depth Interviewing. 
As a qualitative research method, in-depth interviewing represents a constructivist position on social life and on social worlds (Shibutani 1955; Denzin 1994; Josselson 2013). In-depth interviewing is an interactional method for understanding how individuals perceive one or more aspects of their social lives - how they attach meaning and, ultimately, how they interpret their role in the lived experiences of social interaction. In-depth interviewing is a co-construction and qualitative interviewers share responsibility along with participants for creating, framing, and interpreting meaning to the stories that are shared about social experiences (Hess-Biber 2010; Denzin and Lincoln 2011).

In-depth interviewing and the transcription and coding process that transforms interviews into textualized narrative data is a form of hermeneutics, a knowledge production activity that is part of the science of meaning making (Messer, Sass, and Woolfolk 1988; Saldaña 2012; Josselson 2013). Within the context of this study, indepth interviewing was used to access the experiences of human service professionals who participated in interagency collaborations as framed within crisis response and facilitated by FPP. Data-informed in-depth interviews allowed me to focus on issues raised during the previous focus group interviews and participant observation as well as areas the prior literature suggested as potential sensitizing concepts.

I conducted interviews with 40 professionals from 24 different human service organizations. The interviews ranged in length from 29 minutes to an hour and forty-five minutes with an average duration of an hour and ten minutes. Some of the organizations operated specialized human service programs; thus, the sample included representatives from 35 specifically targeted human service programs. The organizations ranged from 
voluntary and faith-based agencies to local non-profit and for-profit agencies to private businesses to organizations that were local departments or divisions of state and local government. The human service workers I interviewed represented multiple professional disciplines among a local spectrum of human services organizations including law enforcement, the judiciary and courts, mental health, the medical field, specialized programs for substance abuse and sexual assault, education, crisis intervention services, foster care, and concrete collateral services.

\section{DATA ANALYSIS}

Qualitative data analysis involves transitioning from abstract or theoretical concepts to ideas that are grounded in more concrete evidence that emerges directly from the data (Corbin and Strauss 2014). This process requires constantly asking questions and challenging potential answers through constant comparisons. Stage coding was used to determine initial concepts and to differentiate lower-level concepts from categories and to clarify assumptions. Concepts and categories were labeled and provisionally identified pending subsequent supporting evidence from the data.

Participant observation data was collected using jot notes followed by extensive field notes, a procedure recommended for researchers working in a familiar social setting (DeWalt and DeWalt 2011). Field notes were hand-coded prior to conducting analytic induction procedures (Miles and Huberman 1994; Charmaz 2014). I used ATLAS-ti (Muhr 1997) to categorically process coding and to generate memos and themes.

Audio-recorded focus group and in-depth interview data was transcribed, audio coded, hand coded, and categorically processed using a qualitative software package, ATLAS-ti. Once coded, analytic induction procedures were used to determine emergent 
themes, potential hypotheses or explanations, and to refine the theoretical application of existing or new concepts related to interagency collaboration (Gillham 2000; Charmaz 2011).

\section{Validity and Reliability.}

The qualitative approach has been subject to several concerns about validity and reliability of data collection, analysis, and interpretation of the findings (Huberman and Miles 2013; Corbin and Strauss 2014). The richness of detail attained through qualitative methods is often particularistic and localized to uniquely sensitive topics and to specialized populations.

I followed five strategies for increasing validity have been recommended in the qualitative methods literature (Silverman 2005; Hesse-Biber and Levy 2006). First, use of the refuting principle is a strategy for refuting assumptions against the data as the qualitative researcher proceeds through data collection and analysis. Second, the constant comparison method can be used to constantly compare concepts, categories and cases. Third, researchers can increase validity by doing a comprehensive data treatment by incorporating all categories or cases into the analysis rather than focusing and interpreting each one separately or individually. A fourth strategy for increasing validity is searching for the deviant cases or outliers, thereby exploring the deviations, or the counter narrative, in the analytical pattern of findings. Fifth, is looking for opportunities to make appropriate tabulations. Finding ways to quantify the data can further highlight trends, patterns, and deviations as well as strengthen validity.

An additional validation strategy I incorporated throughout the study was an ongoing collaboration with two professional colleagues, both of whom are licensed social 
workers with over ten years of applied clinical experience in numerous human service roles and organizations. This type of strategy has been recommended in the literature on validation of qualitative methods and findings (Hesse-Biber 2010; Huberman and Miles 2013). Both colleagues provided validation reviews of the focus group and in-depth interview questions, the composite case study developed for the findings section in Chapter Five, and the findings described in Chapter Five. Their input was particularly essential for verifying the reliability of emergent themes, and for testing refutation and counter narratives.

My in-depth qualitative research led to numerous findings, some surprising and unexpected, and others that were informed by the previous literature and theory. My study situated power and gender among multidisciplinary human service organizations through the process of interagency collaboration. In the following chapter, I present the findings that emerged through my use of the multiple qualitative methods I have described in Chapter Four. 


\section{CHAPTER FIVE}

\section{FINDINGS}

The research questions explored by the doctoral dissertation research were contextualized within several important considerations. First, previous literature has established that definitions of collaboration can be ambiguous because perceptions about what collaboration means and how it should work have differed greatly (Bardach 1998; Gray 1989; Kagan 1991; Kagan 1993; Sowa 2008; Acker 2012). Second, organizations are gendered institutions; thus, organizational processes such as interagency collaboration must be gendered also (Lorber 1994; Martin 2004; Martin 2006; Acker 2012). Third, gender is in and of itself a social institution which installs hegemonic, power-based relations at every level of social structure and social interaction (Connell 2002). Fourth, and lastly, organizations are complex, rational-legal, bureaucratic entities that both empower and constrain social action, including interagency collaboration (Weber 1978; Gray 1989; Hall 1996; Bardach 1998; Clegg, Hardy, Lawrence, and Nord 2002; Perrow 2002; Perrow 2014).

To orient the findings further, it is important to clarify points of consensus within the existing literature that guided the way I conceptualized interagency collaboration as a locus of reflexive comparison throughout the study. Interagency collaboration as a working concept refers to a process for institutional cooperation, coordination, and service integration based on a mutually beneficial and well-defined relationship between 
two or more organizations, agencies, programs, or professionals that "increase[s] public value by working together rather than separately" (Gray 1989; Kagan 1991; Bardach 1998; Mattessich, Murray-Close, and Monsey 2001). My study of interagency collaboration explored both "soft" skill sets, such as emotional labor, relationship building, and "nurturant" care work as well as the "hard" or traditional business skills associated with the formal professionalism of working collaboratively in bureaucratically-structured human service disciplines (Hochschild 1983; England 1992; Wharton 1993; Kilbourne et al. 1994; Moss and Tilly 1996). Hard skills included documenting human services work, budgeting, hiring and training of logistic tasks, managing data and reporting, negotiating contracts, contingency planning, and other forms of business plans, and outcome tracking, among other skills.

In Chapter Five I present three prevailing themes that emerged from the data. First, when interagency collaboration works well, it is more effective when individuals and organizations establish relationships and share negotiated power. Second, human service organizations are institutions that are gendered in intersectional ways; thus, interagency collaboration, too, was found to be a gendered and dynamic intersectional process situated within systems of power. The lived experience of these gendered and intersectional dynamics suggests that sometimes interagency collaboration is constrained even when relationships have been established and sometimes by the demands and obligations of collaborative relationships. Third, interagency collaboration is inhibited by bureaucratic constraints resulting in commonly experienced barriers across multidisciplinary types of crisis-oriented care work. Bureaucratic constraints are a form of power and they shape hierarchies of power within human services in ways that are 
relative, subjective, and exclusionary. Descriptions of relative, subjective, and exclusionary forms of bureaucratic power will be provided at the end of Chapter Five.

In order to contextualize the organizational landscape of human services and common types of interagency collaborations, I begin by presenting an evidence-based composite case study meaning that the sample case described below was constructed using specific details of multiple client families' cases that were shared during interviews, witnessed during participant observations, or described in client case files. The case study is not meant to be representative of all CPS outcomes; rather, the outcome in this case study is indicative of approximately thirteen percent of CPS cases served (CHFS 2016).

My intentions for the composite case study are twofold. My first intention is to use the case study as a framework for illustrating a family's potential progression through an integrated, multidisciplinary human services system. My second intention is to provide readers with a deeper understanding of an experience of being involved in human services. I will revisit the case study throughout Chapters Five and Six to demonstrate different facets of interagency collaboration. Examples from the case study are not presented as proof of my claims; rather, they will be used to clarify important concepts and organizational patterns of interaction. Two considerations in particular are emphasized; interactions among professionals and their organizations and interactions between professionals and the client families they serve.

\section{Candace's Story}

Candace is a six-year old girl living in foster care. Nine months ago Candace approached her teacher at school and said her stomach was hurting. Her teacher asked 
her what was wrong with her stomach and Candace said her belly hurt because her brother "poked her too hard down there." Her teacher, new and recently hired, was not sure how to respond, but she knew she had to help Candace.

The teacher remembered from her employee training and school policy that she would be required to make a report about Candace's statement to the school's principal and to the guidance counselor. When she met with the principal he informed her that as a mandatory reporter in the state of Kentucky, she was legally obligated to call the Centralized Intake regional phone number for the Department of Community Based Services Child Protective Services (CPS) Branch and officially make a report of suspected child abuse and neglect. He referred her to meet with the school's Family Resource and Youth Services Center (FRYSC), a division of state government housed within the school system, for further assistance with making the call to CPS.

The principal explained that FRYSC workers were available to assist with resolving non-educational barriers to learning and to support early academic success; therefore, they would be best equipped to assist her with making the call to CPS. He asked the teacher to look at their website either before or after meeting with them so that she would understand their role and responsibilities. The teacher set up an afternoon meeting with a FRYSC worker she knew by name and quickly reviewed their website during her planning period before their scheduled meeting.

Candace's teacher met with a FRYSC staff person who provided information about CPS as an agency and explained the process of making a report to CPS. The FRYSC worker explained that CPS is a state-operated, public agency tasked with investigating reported allegations of child abuse and neglect and making informed 
judgments about the substantiation or non-substantiation of those claims. She provided the teacher with the Centralized Intake phone number and explained that an Intake Specialist would ask for information about Candace's statements, Candace's physical appearance and demeanor, and about her family make up and whereabouts. The teacher told the FRYSC worker that she was nervous about how the family would react if they found out she reported them and that she, by herself, did not want to make the call. The FRYSC worker agreed to stay with her while they made the call on a speaker phone in her office.

Together they called the Centralized Intake phone number and waited in queue for over 30 minutes. The Intake Specialist who answered the call asked the teacher and FRYSC worker to provide information about their identity and relation to the alleged child victim, to explain the incident that prompted making a CPS report, and to explain their concerns for the child's imminent safety. Candace's teacher answered the Intake Specialist's questions, told her about Candace's statement and provided information about Candace's parents, including their address and contact information that was on record at the school. The teacher and FRYSC worker were given a confirmation number for the report and the Intake Specialist explained they could follow up on the report, but CPS may not be able to share information about the findings other than if the report was accepted for investigation or not. The teacher returned to her classroom, checked on Candace, and put a note in Candace's backpack for her mother stating that Candace had complained about stomach pain while at school.

Upon entering the report information into the Centralized Intake system, the Intake Specialist noted that Candace's family had an open, ongoing CPS case for prior 
child maltreatment reports including previous substantiations of educational neglect for Candace's older brother, Drew. The Intake Specialist determined that based on the family's prior history with CPS and the serious allegations of suspected sexual abuse, the referral met CPS criteria to be accepted as a report for official investigation. The report was electronically delivered to the CPS office located in the county of residence for Candace's family. An investigative CPS worker, Bill, was assigned to the investigation and he began by pulling up Candace's family's case record in Kentucky's CPS case management database, The Workers Information SysTem (TWIST), and reviewing the case history. Bill later spoke with the family's ongoing CPS worker, Debra, to get the most current information about the family's CPS case status.

According to the TWIST file, Candace was the youngest of two children. Her older half-brother, Drew, was listed as being fourteen-years-old. Candace's mother, Tiffany, and father, Bryan, were described as Caucasian adults who have been married for six years. Drew's father, Steve, was documented as a black, 32-year-old man who has been imprisoned for a drug conviction. According to the TWIST record, Tiffany and Steve had cohabited for six years after Drew's birth before Steve was arrested, indicted, and sentenced to 15 years for manufacturing and selling methamphetamines. Tiffany was 16-years-old when she became pregnant with Drew, and she was 24 when Candace was born. Tiffany works as a waitress, and, her husband, Bryan is 35-years-old and works at a local car manufacturing factory. Tiffany has not had extended family support from her parents or other family members because, as the case file history noted, her parents kicked her out of their home when she became pregnant out of wedlock at a young age and by a black man. Tiffany lived with Steve and then with his parents until she met and 
began dating Bryan. The file also stated that Tiffany and Bryan have been homeless several times and move often within their county of residence. The family continues to experience housing insecurity, but so far have avoided entering a homeless shelter.

The ongoing CPS worker, Debra, informed Bill that Drew, Candace's brother, has struggled with anger management and behavior problems and he has a learning disability that was diagnosed in elementary school. Drew and his step-father, Bryan, do not get along and argue regularly. Debra shared that in her opinion Bryan resents Tiffany's prior inter-racial relationship and directs his resentment towards Drew, a bi-racial troubled teen. Drew has consistently been reported to CPS and to the Courts for truancy by the schools he has attended. He is often late for school, misses school, or leaves early without consistent or legitimate parental excuses. Drew's behavior has also been concerning because he regularly babysits his half-sister, Candace, while Tiffany and Bryan are at work. Drew has left Candace unsupervised on at least two occasions as reported by Tiffany and Bryan during monthly home visits. Debra shared her suspicions with Bill that Drew has left Candace unsupervised more often than twice. She does not believe the family has been honest with her about their finances or their disciplinary practices with Drew. They have been court-ordered to take Drew for a psychiatric evaluation and to take parenting classes in the past, but they did not "follow through."

Bill, the investigative CPS worker, continued his investigation into the statement Candace made to her teacher by going to Candace's school and interviewing the teacher, the principal, and the FRYSC staff person involved in making the referral to CPS. Bill then asked to interview Candace. Her teacher brought Candace into an empty classroom and left her with Bill. Bill asked Candace if she wanted to do a puzzle with him, and, 
while casually talking, he explained he was there to talk to her about her belly aches. Candace shared that her belly was not hurting today, but it had hurt some yesterday. When asked what made her belly hurt, Candace repeated that her brother pokes her too hard sometimes "down there" and pointed between her legs. Rather than continue to interview Candace on his own, Bill decided to make a referral to a local Child Advocacy Center (CAC). Per CPS policies and state law, KRS 431.600, Bill knew that a CACtrained forensic interviewer would be better equipped to interview Candace because of her age and the sensitivity of sexual abuse allegations. The CAC could further provide supplemental support to Candace and her family as he worked to determine if Candace had been sexually abused by her brother, Drew. Bill made the referral to the CAC after leaving the school and returning to his office.

At the time of Bill's investigation, Tiffany and Bryan were renting a two-bedroom home in a rural section of the county. They had recently moved there after getting evicted from their previous apartment because of several months of unpaid rent. Bill arrived at their current residence and found Tiffany at home. Bryan was still at work. Bill had planned the visit for a time when the children would still be at school; however, he saw Drew was at home instead of at school. Bill introduced himself and explained he was investigating a report that had been made to CPS regarding Candace. Bill observed that Tiffany did not appear to be surprised by his visit. He asked to speak with her alone and then with Drew alone. Tiffany agreed and Drew went into a back room. Bill asked Tiffany if Candace had recently complained about her stomach hurting. Tiffany said yes and said she knew why. Bill asked her to explain, and Tiffany said she had come home early from work the previous week and saw Drew leaving Candace's room in a hurry. 
She went in to check on Candace and saw that Candace was laying on her bed only wearing a shirt. When Tiffany asked Candace where her other clothes were, Candace said Drew told her she needed to take a bath and change clothes before Mommy got home. Tiffany thought this was unusual, but she had been too afraid to ask Drew about it, and she had not said anything about it to her husband, Bryan, because she was afraid of "what he might do to Drew." Bill asked if Tiffany thought Drew could have touched his sister in a sexually inappropriate way. Tiffany began crying and nodded, yes.

Bill asked to speak to Drew alone. Tiffany left the living room after Drew came in. Bill tried to speak with Drew, but Drew would not look at him or speak to him. Bill relayed the story his mother had shared about seeing him coming out of Candace's room the previous week. Bill asked Drew if this had happened. Drew nodded. Bill asked if Drew had undressed his sister. Drew nodded. Bill asked if Drew had touched his sister in ways he should not have touched her. Drew nodded. Bill asked Drew if he had hurt his sister. Drew shrugged and whispered, "I'm not sure." Bill asked if Drew could tell him the details of how he had touched his sister. Drew shook his head indicating no and began to shake and cry. Bill told him he could go back into the other room. Tiffany returned, still crying.

Bill informed Tiffany they would need to do a Prevention Plan and a Safety Plan pending a decision of the CPS investigation into Candace's statements of being touched by her brother, Drew. He further explained the need for Tiffany and Bryan to meet with the Child Advocacy Center as part of the CPS investigation. Bill retrieved both forms from his briefcase and talked through the CPS agreements with Tiffany for ensuring Candace's imminent safety. Bill reviewed the action plans and repeated that Candace 
could not be left alone or unsupervised with Drew, that Drew had to attend school on time every day unless sick or injured, Candace would be taken to the Child Advocacy Center for a forensic interview and for a medical evaluation of potential sexual injury, and that Tiffany would be required to follow any and all recommendations made by CPS, the Child Advocacy Center, and the Courts or any other human service agency that became involved with the family. Bill cautioned that in many CPS investigations children are removed and placed in foster care if their safety in the home could not be ensured and maintained. Tiffany, still crying, agreed to do anything asked of her to keep her children at home and signed both forms.

A Child Advocacy Center (CAC) case manager, Lori, contacted Bill the next day after receiving the referral for Candace and her family. They discussed the family's CPS case history and Lori confirmed an intention to coordinate an intake meeting with Tiffany, Bryan, and Candace. Bill agreed to be available for the CAC's multidisciplinary team meetings following Candace's intake and forensic procedures.

Bill also decided to make a referral to the Family Preservation Program (FPP) because of the imminence of the safety concerns he had about Candace given her vulnerable age and the seriousness of the sexual abuse allegations as well as Drew's truancy and behavior management issues. Bill completed the three-page Universal Referral Form for Services (CHFS 2016) and submitted it to his supervisor for review and approval. He documented several reasons for referral on the form including the sexual abuse allegations; a lack of appropriate supervision for Candace; Drew's anger and behavior management issues, and ongoing truancy; the need for parenting education; the need for consistent mental health services; and the family's history of housing 
insecurity. His supervisor authorized the referral and forwarded it to the regional CPS gatekeeper, a clinical associate responsible for reviewing and assessing the urgency of FPP referrals received from multiple CPS county offices. The gatekeeper determined that Candace and Drew met the FPP criteria to be considered as "targeted children" or "potential removals" (PRs), a CPS status indicating that one or more children in a home are at imminent risk of removal from their biological parents, thus the family was in need of intensive crisis intervention services to ensure child safety, and, if possible, to preserve the family unit. The gatekeeper emailed the director of FPP to check on the availability of an opening for Candace and her family and faxed the referral form to the FPP director's assistant.

By the end of the next day of Bill's official investigation, FPP services had been initiated with Candace's family and an intake appointment with the CAC had been arranged. Bill had accompanied the FPP worker, Stella, to meet with Tiffany and Bryan for the initial home visit. He made the initial introductions and helped explain the purpose of the referral to FPP. Tiffany was receptive to FPP, an intensive in-home crisis intervention program that would, as Bill and Stella had explained, require eight to ten hours of direct face-to-face contact with the family primarily in their home during three to five visits each week. Bryan did not want to participate in the program and stated that Tiffany could do whatever she wanted "since it was her son that caused the problem." Bryan left the house, angry, and drove away. Bill left soon afterwards while Stella and Tiffany were filling out the FPP-required intake paperwork including a service contract and a release of information that would permit Stella and Bill to openly discuss the progress of the FPP services without Tiffany or Bryan being present. 
The following week Bill attended a Multidisciplinary Team (MDT) meeting at the CAC. The MDT consisted of the CAC case manager, Lori, Bill and Debra representing CPS, the contracted CAC-trained pediatrician, nurse, therapist, law enforcement officer, and forensic interviewer. Lori described her initial contact with Tiffany, Bryan, and Candace during their scheduled intake meeting. Lori relayed that Tiffany and Candace had been talkative and receptive; however, Bryan was reluctant to engage in conversation with her and made comments about how angry he was, particularly with Drew. Lori shared her concerns about the family safety, especially for both Candace and Drew's safety in the home, but also for Tiffany's safety. She wondered about the potential for domestic violence. Lori also conveyed the schedule for Candace's forensic interview and forensic medical exam which would take place in two days. Bill shared a further update with the MDT stating that FPP was working with the family and would be conducting intensive and frequent in-home visits with the family over the next four weeks.

The MDT convened again on the date of Candace's forensic interview. They reviewed the case history again, and then spoke briefly and casually over coffee in the video surveillance room while the forensic interviewer was setting up in the childfriendly interview room. Lori, the CAC case manager, watched for the interviewer's "ready" signal and then went to the family waiting room and escorted Candace into the interview room. The forensic interviewer introduced herself to Candace and asked her if she wanted to play. Candace heartedly agreed. Candace was given a choice of toys, puzzles, crayons and paper, or markers. Candace laughed, excited by her choices, and pointed to the markers. She began to draw and the forensic interviewer joined her in drawing flowers and trees. While drawing the forensic interviewer asked casual 
questions and Candace answered with shrugs, nods, and brief statements. She was very focused on her drawing.

The MDT members watched their interactions on the video monitor in the observation room while the interviewer began to ask specific questions about Candace's family and her interactions with her brother, Drew. Candace began to describe the ways Drew would touch her when he watched her while Mommy and Daddy were at work. She said that sometimes Drew took off her pants when they were playing so he could tickle her belly and her legs. Candace frowned and said she "didn't like his poking stick" because it made her belly hurt and "it didn't feel so good." The interviewer asked if Candace could draw a picture of the poking stick. Candace found a blue marker and drew a long blue skinny shape then she took the yellow marker and drew a broad and jagged triangular shape at the end. The interviewer asked if Candace could draw a picture of herself with the poking stick. Candace drew a picture of herself laying on a small bed with the blue stick between her legs. In her picture she was only wearing a shirt and was crying. The interviewer asked if Candace could tell her a story about her picture. Candace explained how Drew would take her pants off and she would lay on the bed and then he would use the poking stick to tickle her between her legs, but instead of making her laugh it would make her cry. The interviewer asked her how many times Drew had tickled her with the poking stick. Candace, still working on her drawing, said "a bunch." The interviewer thanked Candace for playing with her and, after several more minutes of drawing in silence together, she gave another hand signal. Lori rejoined them in the interview room and began drawing with Candace while the interviewer stepped out and entered the observation room. 
The MDT members reviewed the video-taped interview with Candace and asked the forensic interviewer what she thought the poking stick Candace described could be because the picture did not show up clearly on the video tape. The interviewer said she thought it looked like a broom. The MDT members agreed that Lori and the CACtrained therapist would meet with Tiffany and Bryan to discuss Candace's forensic interview and see if they had an item like the one in Candace's picture in their home. They also agreed to reinforce the recommendation for Candace to receive a forensic medical pelvic examination to determine the extent of any physical trauma and to confirm evidence of sexual abuse. The other MDT members agreed to wait in the video surveillance room while they met with Candace's parents and the forensic interviewer rejoined Candace in the observation play room so that Lori could accompany the CAC's therapist. They met with Tiffany and Bryan in a separate meeting room, and described the item Candace had drawn and her story. Bryan said he had no idea what the item could be, but Tiffany began to cry and shakily shared that the item sounded like a broom she was looking for the previous week. She kept it in the kitchen and had thought it was odd when she found it leaning against the wall in the room where Candace sleeps. Tiffany and Bryan appeared silent but willing, and nodded in agreement to the recommendation for Candace to receive a forensic medical exam before they left the CAC.

The CAC's pediatrician and forensic nurse set up the child-friendly medical exam room after Lori and the CAC therapist affirmed the parents' authorization to conduct the pelvic exam on Candace. Lori led Candace to the Teddy Bear Room on their way to the medical exam room. Lori shared that Candace could pick out any teddy bear she wanted 
and hold the bear during her doctor's visit. Candace picked out a large plush purple teddy bear wearing a floral bow and thanked Lori for her new toy. Lori took her to the exam room and stayed with her until she seemed comfortable with the pediatrician and the forensic nurse. Lori left and rejoined the other MDT members while they waited for the results of the forensic medical exam. Forty-five minutes later the pediatrician entered the room and explained the preliminary results of Candace's pelvic exam. Candace had scratches and tears in multiple places along the periphery of her vaginal wall consistent with being penetrated by a thin, long, blunt object. She also had a buildup of scar tissue that indicated advanced healing of multiple past penetrative incidents. In the pediatrician's professional medical opinion, the physical evidence was consistent with Candace's story and the drawings Candace provided during the forensic interview. The MDT members signed the CAC case notes and evidence collection forms before concluding their meeting. Lori agreed to make copies to share with all the MDT members. The pediatrician, law enforcement officer, Lori, Bill, Debra, and the CAC therapist met with Tiffany and Bryan to explain the findings of the medical exam and potential next steps.

Although upset and shaken, Tiffany and Bryan were able to coherently ask questions in response to the news of the medical findings. They asked if Drew would be arrested. The law enforcement officer explained that because Drew was a family member and a minor, their family's case would fall within the jurisdiction of Family Court and, potentially, the Department of Juvenile Justice unless a psychiatric evaluation of Drew determined the causes of his actions to be associated with a mental health diagnosis. They asked Bill and Debra if Candace was going to be taken away from them. Bill 
explained that he would be substantiating the allegations of sexual abuse and at that point the case would revert back to the ongoing CPS worker, Debra. Debra further clarified the need to staff the case with her supervisor prior to further official decision-making about Candace's status in the home. Tiffany asked how soon they would know. Debra said she was unsure, but that the ultimate decision would be up to the county attorney and the Family Court judge officiating over their court case. Bryan, very angry and still upset, turned to Tiffany and said he could not go home and "look at Drew." He said, "I don't know what I will do to him if he's close enough for me to get my hands on him when he gets home from school today." Tiffany began to cry harder, and the law enforcement officer asked Bryan if he was making a threat towards Drew's life. Bryan shrugged, shook his head, and looked down. Tiffany informed the group that the FPP worker would be coming to do a home visit soon and they needed to leave. The group dispersed after saying farewell to Candace and telling her how well she did today. They told her she was a "strong little girl." Candace hugged her teddy bear close as she left with her parents.

Bill and Debra met with their CPS supervisors upon return to the CPS county office. They had spoken in the car on the way back to the office and agreed they needed to recommend filing for an emergency removal order for both Candace and Drew to be placed in foster care due to imminent safety concerns. Their supervisors agreed and Bill, as the primary worker due to the CPS investigation, called the Family Court judge's office to request the emergency order. The judge granted the order and her assistant told Bill it would be ready to pick up in thirty minutes. Bill contacted the State Police office and asked for a police escort to accompany him during the removal of the children. 
Meanwhile, Debra had entered the children's names into the statewide database for private foster care agencies because the state-paid foster homes in their county and the surrounding counties were filled with no immediate availability. They were contacted by two local agencies with openings. Debra accepted one agency's opening for Candace and the competitor's opening for Drew because he needed a therapeutic placement due to his behavior management and mental health needs. The available home also had motion sensors in place for sex offenders and for minors with the potential to behave violently.

Bill picked up the judge's removal order at the courthouse after calling and coordinating the arrival time for the removal with the State Police. Bill also texted the FPP worker and informed her that the removal of Candace and Drew preceding their placement into OOHC (out-of-home care) would occur during her home visit with the family that evening. Bill and Debra arrived separately and remained in their cars for a few minutes waiting until the State Trooper arrived. They knocked on the door and when Tiffany opened it she nodded in immediate understanding and began to shake and cry. She explained that Bryan had left after dropping her off at home, but she would call him so that he could say good-bye to Candace before she was removed. The FPP worker, Stella, asked Tiffany how she could help her. Tiffany asked if she would play with Candace while Tiffany bagged up some of her clothes and toys. Tiffany, crying hard, took several plastic bags from the kitchen. She handed several bags to Drew and told him to go get this "stuff." Bill and the State Trooper went with him to monitor his safety and his progress. Debra observed Tiffany as she packed Candace's clothes and some of her favorite dolls and toys. Tiffany hugged Drew and told him she would see him soon. He walked out with Bill and got into Bill's car. Tiffany asked if Debra could wait until 
Bryan got home before taking Candace. Debra said she could stay for fifteen more minutes. Tiffany called Bryan, but only got his voice mail. Candace asked Stella, the FPP worker, why her Mommy was so sad. Stella patted her on the arm and told her everything would be fine. Debra, the State Trooper, and Stella walked out with Tiffany and Candace after fifteen minutes had passed. Bryan returned as Tiffany was loading Candace into Debra's car. He began to run towards them and the State Trooper asked him to stop and wait where he was and then approached him to talk. The State Trooper told him to calm down, and, after a few minutes when he was more composed, he directed him to approach Debra and his family slowly and calmly. Bryan knelt down and hugged Candace, told her he loved her, told her everything would be fine, and Mommy and Daddy would see her soon. She nodded and said, "I love you, Daddy." He responded, "I know, baby. I love you, too." He turned to Tiffany, held her while she cried, and they went into the house and shut the door. Debra handed Candace her new purple teddy bear she had gotten at the CAC earlier that morning to hold onto before they drove away.

FPP closed their case following the removal, and the Family Court scheduled the adjudication for the CPS sexual abuse case on Candace for the following week. The Family Court Judge ordered Candace to remain in foster care pending Tiffany and Bryan's completion of a 12-week parenting education program, completion of psychiatric evaluations for all family members, Drew being admitted into and completing an inpatient juvenile sex offender treatment program, and all family members beginning and consistently attending individual and family therapy. Subsequently, Tiffany and Bryan were evicted from their home because they used their rent money to pay for parenting 
classes and two of the four court-mandated psychiatric evaluations. While they were staying in the Salvation Army's homeless shelter, Bryan slapped Tiffany during an argument. She left and moved into a local domestic violence shelter. Tiffany and Bryan separated soon afterwards. Over the last nine months since Candace and Drew were placed in separate foster homes, Tiffany attended several Family Court review hearings and became involved with several additional human services, one of which transported her to a local women's clothing closet so that she could receive donated items of professional clothing more suitable for attending court. Tiffany continued to attend regularly scheduled supervised visits with Candace and Drew in a secure, video-taped observation room at the human service organization where she also participated in parenting classes and in a domestic violence support group. She moved in with a cousin in a run-down apartment, but told Debra her part of the rent was reasonable and the landlord was nice, although he refused to pay for recent bedbug treatments throughout the home. Being unable to pay for bedbug treatments had prevented her from getting inhome unsupervised visitation with Candace.

Bryan moved to another county and had no further contact with Tiffany or the children. Candace began seeing a therapist at a local sexual assault treatment center on a weekly basis, but Drew continued to have problems at school and had to be moved to two other therapeutic foster homes because of his anger and beyond-control behaviors. $\mathrm{He}$ continued to await an opening in the closest in-patient sex offender treatment program. At her last meeting with her CPS worker, Debra, Tiffany was informed that concurrent case planning had begun and, if the children remained in OOHC for 15 months or longer, termination of parental rights would be pursued with the goal of legal adoption for 
Candace and Drew. Tiffany no longer cries. She nodded and said she understood. During supervised visits, Candace started calling her mother by her name, Tiffany, and referred to her foster mother as "Mom." Candace and Drew may never go back home again.

Candace's story represents a composite case study scenario meaning that while they are not one actual family, the details of their case happened to multiple children and their families in multiple cases. The case progression for Candace's story was constructed from descriptions provided during the focus group and in-depth interviews I conducted, from case file reviews, and from the human services I observed during numerous participant observations across multiple organizational settings. Gaps in the case progression were filled in with details from my own professional experiences in human services which spans eighteen years.

The composite case study was constructed as a means to demonstrate the immense, and often urgent, need for human services as well as the many essential ways that human service organizations intersect, collaborate, become integrated and interdependent, and, in many ways, interlock. Candace's story further demonstrates the amplified commitment of human service professionals in response to family violence and child victimization, particularly child sexual abuse, and the intensity of professional relationships that often emerge from the experience of sharing crisis care work (Cancian 2000; Cancian and Oliker 2000; Hugman 2014).

The composite case study provides situational context for the dissertation findings; however, further context is needed to gain an understanding of how I arrived at the emergent themes, analyses, and interpretations presented Chapter Five. The 
qualitative methods I used for the dissertation research were informed by and built upon the results of a previous study wherein a survey was administered while interning at a regional Child Advocacy Center during the Fall Semester of 2013. In the next section I briefly review the major findings from the Interagency Collaboration Survey (Moore 2014) that inspired me to continue digging deeper into the gendered, intersectional, organizational complexities of interagency collaboration.

\section{Contextualizing the Previous Study Findings}

Many of the qualitative findings presented in Chapter Five either confirmed or richly supported the quantitative findings of the Interagency Collaboration Survey that I constructed and administered during the previous applied doctoral internship study (Moore 2014). The survey findings that were produced using an exploratory factor analysis indicated that multidisciplinary team members collaborating within the context of a regional Child Advocacy Center were motivated to engage in collaborative exchange when they achieved positive outcomes and shared good communication related to a sense of enhanced work. The pros and cons of their team collaborations included enjoying collaboration and seeing it as a good use of time; however, as necessary as interagency collaboration was viewed as being, effective collaboration was perceived as difficult to achieve. Other factors such as lack of motivation or buy-in appeared to be related to a preference for focusing on one's own job. Thus, even though collaboration was viewed as a way of making work easier, the process could be too slow. Poor motivation was also related to a desire to change their agencies' collaboration models.

Additional survey findings indicated that the importance of collaboration was related to strengths in the areas of openness and familiarity with an emphasis on 
receptiveness and interest during facilitated, local forms of collaboration. Gaps in collaboration were related to challenges in solidifying a collaborative foundation as the basis for a mutually beneficial and practical process of collaboration characterized by similar, but complementary services. Lack of reciprocity, trust, and communication were identified as further challenges. Competition for funding was also identified as a challenge to effective collaboration.

As interesting as the survey results were found to be, the Likert Scale responses were general and non-descriptive of personal attitudes, lived experiences, standpoints, or other interpretive meanings, criticisms consistent with the use of many types of quantitative methods (Singleton and Straits 2009). The findings did demonstrate statistically significant differences in perceptions of motivations, strengths, and gaps related to engaging in interagency collaboration; therefore, I used the previous survey findings to construct the focus group and in-depth interview qualitative guides as I adopted a qualitative methods approach to the dissertation research. I also relied upon the survey findings to sensitize me to motivations, strengths, and barriers expressed during participant observations and interviews. As a result, I gained a greater awareness of the richness of participants' responses about their lived experiences of interagency collaboration. One theme in particular that strongly emerged from the data was the meaning and value of establishing collaborative relationships, both within organizations and across agency divides.

\section{RELATIONSHIPS AND INTERAGENCY COLLABORATION}

Previous scholars have emphasized the necessity for interagency collaboration within human services as a best practice approach for prevention, but also as a framework 
for enacting ethics of care (Gilligan 1993; Hugman 2014). Although interagency

collaboration is an engagement strategy for integrating multidisciplinary human services, this approach has also been viewed as conflicting with traditional, hierarchal bureaucratic modes of efficient and productive organizational work (McAllister and Dudau 2008).

Definitions and perceptions of collaboration are shaped by gender and gendered organizational processes; thus, the creation of more "communal" environments through which collaboration regularly occurs may be perceived as too feminine or too personal and in opposition to professional, objective, task-oriented work commitments (Coleman and Rippin 2000; Kanter 2008). Interagency collaboration, then, is framed by many human service workers as normal and essential, but concurrently challenging and inefficient. As a licensed family therapist explained:

You've got to collaborate in a way where people can do their jobs and not be told how to do their jobs, and then you work together for the good of the client. The problem with collaboration is everyone comes into it with different motives.

The therapist emphasized the need to maintain professional autonomy and organizational boundaries as parts of collaborative exchange and further commented that different professionals enter collaborations with different motives and agendas. Meanwhile, a psychiatric practitioner shared a different perception of interagency collaboration in her statement:

It's more like integrative health care. You have the idea of somebody from every discipline participating in patient care. So, you have social work, you have therapists, you may have providers, you have peer support..., I mean, just kind of a whole gambit of people working together to make sure that a particular patient is reaching their best potential and obviously staying safe. 
Both the therapist and the psychiatric practitioner discussed the need to work together and collaborate; however, their differing views illustrate why finding common ground and understanding could be professionally challenging. The therapist emphasized the need to collaborate while concurrently maintaining professional autonomy, and he further described how potential conflicts could result from having different organizational motives or agendas. Somewhat differently, the psychiatric practitioner's conceptualization of interagency collaboration was more integrative and focused more on meeting client needs rather than on divergent organizational motives.

The next two accounts further illustrate divergent viewpoints about the meaning of interagency collaboration and what it looks like for each of the participants individually. Their comments also demonstrated gendered conceptualizations of interagency collaboration. A career law enforcement officer commented:

[Collaboration is] problem solving.... It's getting people in the same room together..., but it has to be entrenched in a process. Somebody has to have responsibility for arranging it, but if you just have a group of people who want to collaborate because personally they get along, that's fine for as long as those people are in those positions, but they have to become ingrained in some way to sustain that type of collaboration.

His comment not only offers a different perspective of what collaboration entails, but is also focused on the rational management of and accountability for the collaboration process from a problem-solving perspective. This description of collaboration seems to be hierarchal and emphasizes the need for leadership or management, the need for someone to be in charge to ensure consistent coordination and scheduling. His account further explains that collaborations may not be sustainable because the professional members change. Liking each other may not be enough; therefore, there has to be a 
purpose for collaborating beyond personal relationships. Somewhat differently, a family

law attorney described her concept of collaboration by stating:

I always hope that everyone [in a collaboration] will be professional, ethical, and honest, so those would probably be my first, fundamental expectations of any relationship with my partners. Also, follow through..., when you say you're going to do something, make sure it's done. Also, to be patient. One of the things with all of our agencies working together whether it be legal aid or the health department or the Cabinet for Health and Family Services or [name of a domestic violence shelter], so many of us have so many people who we're trying to help and want to get everything done yesterday, but there's only 24 hours in a day. So trying to be mindful of that and what the demands of my fellow professionals are, but also them being understanding that sometimes a court system doesn't wait on us, and that we have to get things done under certain deadlines, and, for the most part, there's a real spirit of professionalism and cooperation.

Her conceptualization of interagency collaboration was also individualized, but was much more relationship focused and inclusive of other types of multidisciplinary, "cooperative" professions. She also acknowledged the similar bureaucratic work demands that partnering professionals face. Her account seems more relationship oriented, or feminine in orientation, while the previous account of the law enforcement officer seemed more work oriented, or masculine, with a focus on efficiency and rational problem-solving. As collaborative as this vision is, there are still deadlines, such as those imposed by judges and state law, that limit the extent and quality of interagency collaborations that can occur.

All of the quotes thus far represent diverse ways in which different organizations, differing disciplinary backgrounds, and differently trained professionals conceptualize and approach interagency collaboration as a process and their expectations of interprofessional relationships. While only a few participants openly talked about not enjoying participating in collaborative exchange (discussed below), the vast majority of 
study participants and human service professionals I observed and both formally and informally interviewed expressed avid interest, sincere motivation, a sense of obligation, and personal commitment to the necessary and beneficial process of interagency collaboration as an integral part of their human services work.

The importance of establishing cross-organizational and cross-sector relationships within human services cannot be overstated (Bardach 1998; Gray 1989; Kagan 1991; Kagan 1993; Sowa 2008). As a mental health service coordinator framed it, "We're in the trenches together." Human services intersect with numerous disciplines and many disciplinary philosophies as well as multiple social institutions including government agencies and programs, the educational system, the criminal justice system and law enforcement, and the medical and mental health system, all of which are operating, sometimes competitively, within an economic environment of public, private, for-profit, or non-profit funding. The outcome is a vast collective of formal and informal complex organizations and organizational processes, including interagency collaboration (Martin 2006). Collaborative ventures are embedded within the institutional structure of human services and are unavoidable, essential, interpersonal, and enculturated as normal procedural practice. When defined as part of "work" though, collaboration can add to the strain of the everyday stressful work routine of providing human services; therefore, relationship building may be a vital strategy for offsetting intense professional strain.

\section{Establishing Relationships}

Every participant interviewed emphasized the importance of building collaborative relationships. Having relationships made initiating and participating in collaboration easier and more effective. Established relationships motivated participants 
to engage in interagency collaborations, and a lack of professional relationships was

occasionally mentioned as a reason for poor collaboration. Referring to the problems that new human service workers commonly encounter and the complications their lack of established relationships with families and in the field can create, one FRYSC worker said:

Social services has a lot of new staff. The ones who have been there for a while know that we have a lot of background with the families. So sometimes, if they just hired the new ones, they say, "Oh, we can't talk to you." Because we usually share with them what we know and they share with us. So, if they're not going to share with us, then.... We have a good relationship with those people, the old workers know, but the new workers go to the guidance counselor instead of coming to us. They probably think she's in on it, but she don't know.

By going to the guidance counselor, a professional trained to focus on meeting the educational needs of students rather than FRYSC workers who are tasked with meeting the non-educational, personal needs of at-risk children and families, essential time and critical information may be lost. Professional relationships among different agencies allow human service workers to know whom to call, the roles of various professionals involved, and which professional has the expertise needed to "fit" the crisis situation at hand. The interworking of human services, including building a network of professional contacts and navigating the landscape of a local community of ever-changing organizations, programs, and staff can be a very steep learning curve for those new to the profession. For example, a judicial case manager observed:

Turnover is high. Turnover at the Cabinet is high. Turnover in non-profits is high. Turnover in therapists who work for therapeutic foster homes is high. I don't know why. I've never worked for one of those agencies. It must be a very stressful job because turnover is really high, and the way those contracts are set up, they're only supposed to us therapists within 
their company, and, so sometimes kids go without services because they're in the process of hiring a new therapist, and then they get that therapist in and that delays sending them home because it delays their report [to the court]. They have to be comfortable enough to write a recommendation. You need the same therapist or they might not get to go home.

Her description illustrates how a high rate of turnover produces instability and consistent flux within a service community that relies upon the ability to build and maintain relationships with knowledgeable professionals equipped to deal with a crisis-oriented service population. Although high turnover was viewed as problematic, yet normal, within the stressful ecology of care work, a vast majority of participants voiced concerns related to the rate at which they encountered new workers.

The few accounts wherein human service professionals expressed frustration or concerns about collaborative relationships, as in the previous account, were highly contextualized and situational. For one participant, a law enforcement official who had specialized in child sexual abuse cases, maintaining established relationships, while admittedly necessary, was described as sometimes uncomfortable and overwhelming. $\mathrm{He}$ commented:

The role of the police is different from the role of any of these other people and sometimes we're really hard to collaborate with. We're used to being in charge, and somebody else sticking their nose in is kind of an uncomfortable thing for us. We're not going to stop them from doing their job, but, when they want to have input on how we do ours, it's hard to make them understand sometimes because it really is different. The best thing about it was also the worst thing about it. When I was working sex abuse, I developed really good relationships with all the social workers. I knew them all by name, I had their numbers in my phone, we worked really close, we were a family, but that's also why after two years I said I was either going to quit this job, or I was going to promote out of it, or I was going to jump off the roof. It was bad. It was like I had eight wives. They were chasing me all the time. They had a few cases. I had eight counties worth. Their emails were coming in at such a rate it was hard to manage. It was too much. 
His description demonstrates how collaborative relationships may be fostered under extraordinary crisis conditions, and how those same caring commitments can take on heightened degrees of social pressure, especially within expectations for professional reciprocity. According to this participant's experiences, the best parts of collaboration, working in concert to achieve the critical goal of child protection, had the potential to further inundate an already overwhelming workload. His account further illustrates how a hierarchal approach to an authoritative role may be an impediment to collaboration. Like most professionals involved in protecting children, he described how how was overly burdened with extraordinarily high caseloads; thus, collaboration can be impeded by differing views on authority as well as a lack of resources and "go to" professionals.

Another participant, a drug court worker, described her experience of trying to establish relationships while starting up a new drug court program. Her account illustrates how turf issues, distrust, and apprehension about new agencies or programs within a small-town local community may sometimes inhibit communication, collaboration, and openness to building new relationships. She commented:

When we first started working with the Cabinet, social services, it was automatically awkward because their job is to protect the children or take the children, and our job is to try to get them reunited if that's what they want or if that's what can be. So, absolutely, I was like the enemy at first, and it took getting a couple of the case workers just to acknowledge us by providing them with information, but yes, the Cabinet at first, it was like, "No way am I talking. What can you tell me that they're [client families] doing wrong?" It's not always perfect with the Cabinet. That's probably our hardest agency [to collaborate with], but it depends on the specific worker and [name of county] is easier to work with than [name of a different county].

This worker's account demonstrates the situational complexities that may emerge when different disciplinary philosophies, organizational goals, types of agendas with client 
families, individual personalities, regional geography, and local cultures converge. The potential result is resistance or refusal to engage in relationship building which inhibits interagency collaboration.

For some agencies, though, building relationships was a critical part of their startup process. Similar to the demands on new worker, new agencies or programs also benefit from building collaborative relationships. The establishment of new non-profit charitable organizations that can contribute to meeting specific family needs is common throughout the United States and this region of Kentucky (Forbes 2012). The growth of national food pantries to help meet the nutritional needs of low-income individuals and families is a prime example of the ongoing changes in the field of human services. But, for an organization to be successful, it must work at marketing itself and establishing relationships among workers who provide necessary referrals. For example, a coordinator of a voluntary clothing donation service said, "Once relationships were formed, they [local agencies] began to use me and refer to me." Another participant, a director of a non-profit family services agency, shared a similar sentiment when he said, "Don't just go in as a social service provider, go in as someone who's there to build a relationship and then all those other things come along easier." Likewise, a focus group interview participant commented:

We all have a pretty good attitude. You know what I mean? So that goes into building that relationship with the people you're trying to collaborate with..., I mean, not necessarily being friends with them and going out on the weekends with them or anything, but having a good working relationship.

A vocational rehabilitation coordinator similarly described her relationship with another agency professional: 
It's much easier to partner or collaborate with somebody that you are on friendly terms with, like the veteran guy over at OET [Kentucky Office of Employment and Training]. He's awesome and we're really friendly with each other. He's enjoyable to talk to, and when you have the personal relationship to an extent, like a good co-worker relationship, it's easier to talk to them. It doesn't feel as formal. You can just go to their office and be like, "Hey, I got this guy and this is what's going on." It makes it [collaboration] easier.

Establishing relationships, as explained by these participants, ensured a continuous flow of referrals as well as a strategy for sustaining positive working relationships. Positive relationships not only enhanced their collaborative experiences, but also expedited human services for client families, a sentiment further described by a police administrator. She said:

It's the effort that you put into relationships. In terms of relationships and building relationships, that is important to me and those do work in my favor, and so maybe that is an extra effort that some people make or don't make, it may help or it may hinder sometimes because sometimes you have to back away, and go, "Whooh, I'm too close." And things are going to happen that are out of our control and you just want to go, "Are you kidding me?" But then you get on the phone or you go over and you say, "Hey, let's talk about what we can do and what's available" type of thing because, ultimately, it's not about us [individually]. It's just about working together.

Building relationships was additionally viewed as a critical approach to navigating the legacy of job transitions after employees leave human service organizations and began new jobs in new human service roles. For example, an agency director commented:

In the eight years I've been here I probably have eight to ten different people that I've supervised who now work as a social worker for either the state of Kentucky or the state of Tennessee somewhere, and I still have good working relationships with all those people to where I can pick up the phone, and maybe I don't even call from my office phone to their office phone. Instead I just call them directly on their cell phone and we're able to collaborate about things. 
His statement reveals how established relationships can be a resource for future needs, but also how having relationships can provide a "work-around" option for cutting through the bureaucracy of professional etiquette and traditional modes of communication such as calling an office phone line, leaving voice mail messages, or wading through organizational e-mails. My participant observations, too, supported his statement. I witnessed a vast majority of professionals sending text messages to personal phone numbers rather than calling each other or sending longer e-mailed messages. Once their inter-professional relationships had been established, most of the human service workers I observed seemed to prefer personal text messages.

When they work relationships can provide a foundation of trust, reciprocity, and mutual respect, and they increase the sense of professional obligation to communicate in working together to meet the multiple, and often imminent, needs of crisis-oriented families and children. During participant observations in a multi-county family preservation program setting, I witnessed a mix of personal and professional interaction, both hugs and formal handshakes, as well as sighs and groans of frustration followed by deeply encouraging words of support - all indicators that, indeed, relationships between professionals and their organizations were established and in place.

Thus, according to the vast majority of participants in my study, interagency collaborations work more effectively and outcomes for families are profoundly enhanced when professionals have the opportunity to establish collaborative partnerships. Nonetheless, a barrier to effective collaboration can occur when there is disagreement on how working together should take place and on who is in charge, as well as when the number of professionals who serve as resources is insufficient to meet demands. As 
important as establishing relationships appear to be, opportunities to develop them or to consistently engage in them were often inhibited by dynamic, intersectional, gendered complexities within and between institutions and by bureaucratic constraints within organizational settings.

\section{LIVED REALITIES OF INTERAGENCY COLLABORATION}

The lived realities of interagency collaboration are that it can be difficult to initiate or sustain and sometimes, for a variety of reasons explored in my study, it does not work well. Interagency collaboration is a gendered organizational and interpersonal process embedded within the social structure of gendered human service organizations. Making this claim has three important implications. First, imposing an organizational framework of masculinized bureaucracy upon traditionally feminized caring fields leads to organizational tensions and conflicts that constrain interagency collaboration. Second, formal organizational policies and procedures that mandate the occurrence of or a process for interagency collaboration may not in reality facilitate interagency collaboration. Third, individuals as social actors are the agents through which intersectional power differentials are constructed and perpetuated as part of both institutional and interpersonal practices.

The participant observations, focus group interviews, and in-depth interviews through which I engaged human service professionals provided rich and detailed insights into the myriad ways that interagency collaboration occurs as a gendered, intersectional process. In this section of my findings I will lay out how the human service organizations I studied are gendered organizations and I discuss how interagency 
collaboration is constrained by rational-legal bureaucracy in ways that promote the lived realities of interagency collaboration.

\section{Gender and Other Institutional Intersections}

Gender is reflected in everyday interpersonal interactions, but at a broader level of culture and institutional interaction, gender is a social institution that is embedded in and intersects with other institutions and their hegemonic social structures (Lorber 1994; Martin 2004; Connell 2002). My findings build upon Lorber's descriptions of gender "as an institution that establishes patterns of expectations for individuals, orders the social processes of everyday life, is built into the major social organizations of society, such as the economy, ideology, the family, and politics, and is also an entity in and of itself" (1994, p. 1). Lorber further stated, 'First, no institution 'stands alone,' and, second, gender is done everywhere, 'inside' other institutions but 'outside' them also" (1995, p. 1265). Martin, too, said, "Even if people could leave gender at the door, gender would still be present because it was already there" (2006, p. 255).

Everyday social life is organizationally-driven. Individuals are surrounded by organizations, and, according to Hall (2008), "we are born in them and usually die in them; the space in between is filled with them. They are just about impossible to escape. They are inevitable" (p. 1). Patrimonial organizations have been all but replaced by bureaucratic, gendered organizations (Weber 1978; Kanter 1993; Perrow 2014). Bureaucracies, including human service organizations, are governed by legal-rational methods of operation and surveillance, resulting in highly rationalized modes of operation (Foucault 1977; Weber 1978). As complex and formally rationalized as bureaucracies are, they are not gender neutral, nor are they free of the myriad ways that 
other factors, particularly race, rank, licensure, and region, among others, intersect with gendered modes of operation within a legal-rational setting (Kanter 1993; Britton 2003).

Human service organizations, especially those funded and operated by state and federal government, are vested with legal-rational authority and charged with carrying out decisions based on the rule of law, laws that may pre-empt ethics of care (Gilligan 1993). Human service organizations, though, present an interesting cultural phenomenon because they arose out of an ideology of "separate spheres" that hegemonically shaped societal perceptions about the restricted domains of “women's work” (Newman 2007). Much like the ways in which the home was traditionally viewed as a natural, private, and separate domain for women while men resided in worlds of work, the field of human services emerged as an organizational environment dominated by women who were viewed as inherently natural care takers for individuals - especially other women and children - and families with crisis needs (Coontz 2011). Ethics of care originated from this tradition, but the more bureaucratized human service organizations has become, the more care work has been eclipsed by masculine ethics of justice, or bureaucracy (Gilligan 1993). Candace's story exemplifies reasons why ethics of justice are correspondingly necessary, especially for victims of crimes; however, the case study also reveals ways in which the needs of at-risk families may become peripheral to bureaucratically-imposed systemic mandates and procedures for processing such "cases." Consequently, a major concern articulated throughout my dissertation is the potential loss of scope and focus in the efforts of dedicated human service workers to balance the human element of caring services and their bureaucratic obligations as professionals conducting the business of human services. During two separate interviews with agency officials I asked the 
following clarifying question using phrases from their own statements. "Are you saying the business of human services sometimes gets in the way of actually doing human services?" The first official, who worked within the Department of Juvenile Justice, answered:

Oh, yeah, that's a definitive yes. Policies of agencies are typically written to support the needs and desires of agencies, not necessarily written to support the service provision to the clients, and that's just a natural part of the bureaucracy of any agency whether they're government, or whatever. So, yes, I think so because it is a business, and whether it's a for-profit or not you still have to meet your bottom line in terms of a budgetary standpoint. You still have a governing body that establishes expectations or standards that you have to meet and comply with, so yes. I think it does get in the way sometimes.

While answering my follow-up question the second administrator, a specialized services center director, discussed the minutiae of bureaucracy and further contextualized the ways in which the menial, or the "hard," concrete business aspects of human services compete with direct services to client families. She commented:

It [human services work] is a lot of paperwork and checks and balances. If we have a therapist who's doing therapy today, they break down what they do in 15-minute intervals. So if they take a phone call, that's 15 minutes, then with that client [there is] 15 minutes, and they have to put it [their time intervals] on this sheet of paper and that on that sheet of paper, and that one has to go to the administrative assistant who enters it into our system which then in turn eventually matches their timesheet that's also broken down and takes a long time to fill out, to go back and pull up all your reports to see which funder paid for what and how long you worked on that funder's stuff that day. And, so, I think that it does. Some of the reporting especially, the reporting and tracking of that information can take away from the services that we provide on a daily basis. I mean, I know it does for me.

Both sentiments illustrate how human services work has become increasingly bureaucratized to the extent that a bulk of service hours, meaning both the physical and mental energy of professionals, are invested in accountability measures, budgeting, and 
outcomes tracking that may inhibit the personal commitment and care of human services professionals. A law enforcement official further demonstrated these frictions when he described the demands of trying to focus on one's own role in the midst of collaborative crisis work with other organizations. He commented:

It gets in the weeds because we've got our job to do and you've got your job to do, and, when it comes right down to it, we collaborate to try to make each others' jobs easier, but I'm standing there sort of saying don't let me stop you from getting your job done or whatever, but I've got to get my job done. That's the trick bag that law enforcement ends up in a lot is that we understand our job really, really well, but our job is so hard to keep up with and there's so much stuff coming at us. I think a lot of times other agencies think we're just arrogant and don't give a damn. That's not what it is. We're doing our best just to barely get done what we've got to get done, and to get it done well and right, it takes all of our attention. Many times I can't stop to be touchy-feely and deep down understand what you've got to do, because, man, I've just got to have you handle that 'cause I've got to really get on this over here. I know that's probably the same way they feel, but ours is whether somebody goes to jail for the rest of their life or not. It's a big deal. There can be some friction in there.

The importance of interagency collaboration was further clarified by an agency director who commented:

We ought to be talking to one another because, especially in social services, we're dealing with people's lives..., I don't think Burger King and McDonalds are going to talk to each other. I don't think McDonalds is going to tell Burger King what's in the special sauce, and they're just selling hamburgers. We're dealing with people's lives.

His emphasis on the idea that human services extend beyond traditional business models and proprietary for-profit services demonstrates how the human element of human services is a driving force within care work that potentially can become secondary to bureaucratic demands. A judge framed her expectations of collaborating professionals who represent client families during a similar account:

You love the challenge of trying [to help people] because you're doing the right thing. Make sure people are informed. Make sure people have 
adequate chances to learn about the things we're dealing with, adequate chances to get services, opportunities to provide whatever needs to be provided. If they don't take it [professional help], they don't take it, but you don't want it to be because you didn't try. We're not just going through the motions for the record or to get through the day because these are peoples' lives and they matter. The buck stops with us. If we [judges] don't demand and have high expectations, who's going to? It could get real slack and it could get really bad. We've got to demand that people do what they're there to do whether it's lawyers, cabinet workers, or whoever. They need to play their role and do what they're supposed to do.

The judge's comment further demonstrates how caring about the lives of client families can be such a driving force of motivation that, if need be, may be demanded through authoritative mandates and judicial expectations.

In contrast, one participant strongly asserted that the bureaucratic frameworks and increasingly privatized dimensions of human service systems, while at times constraining, were also ethical obligations entrenched in care. He described how these bureaucratic features of human service organizations make care work possible. The participant, a family counselor, stated:

Can human services be both beneficial and a business? I think it depends on what your definition of what a business is for. Is it wrong to make money? Absolutely not. Is it wrong to make money because people have pain and suffering? No. Religion has done it for thousands of years. Have they made money per se? No, but people give it. It comes back to providing, filling a need. People need to eat. There are places people can go. People need to be heard. People need to be validated. People need help. People need to know they have some choices. People need someone who is an expert. There are ways to guide them in a way that moves beyond their defenses and helps to get to them, people need that, and, if you're good at it, charge whatever you want to charge. People will come. You'll know when you don't have any clients that you're charging too much. This is the ethical thing. Ethically speaking, if I'm a private practitioner I have to have the lights on, I have to have the doors open, I have to be there. I have to have a car, I have to have a home, I have to have all these things because I have a hundred people who come to me for therapy. Anyone who says I don't have an ethical obligation to make money and manage money is flat out wrong. 
His comment illustrates how, for some, the bureaucratic structure of human services is viewed as an ethical necessity that provides a pathway through which individuals with needs can access expert care. His position on the ethics of bureaucracy diverged from the majority of participants who described bureaucratic constraints as limits to their ethics of care; however, this counter narrative is an important demonstration of what has been discussed in previous literature as the ways in which bureaucracy can also galvanize social action and social change (Clegg, Courpasson, and Phillips 2006). Without salaries, mileage reimbursements, administrative budgets, and other monetary incentives, professionals would be unable to provide needed assistance and vulnerable families and children would be at risk. All the same, bureaucratic dictates put enormous demands on professionals to fill out a plethora of forms and to document the minutiae of their work lives which I discuss in more detail later in Chapter Five. Bureaucratic constraints such as these reveal how human services are situated within a contested space, a space occupied by the tensions within intersectional, gendered, organizational processes. Intersectional, gendered, and organizational processes. Ethics of care and justice are threaded throughout human service bureaucracy, but are enacted through intersectional, gendered, organizational processes. Gender, then, as a central organizing feature of everyday social life, is so present that it is often experienced as a taken-forgranted orientation to everyday life as illustrated by many of the participants in this study who seemed to lack consciousness of the role gender played in their personal and professional lives or its embeddedness in the structures of social institutions (Berger 1963; Connell 1987; Smith 1987; Bartky 199;1 Lorber 1994; Acker 2012; Kimmel 2014). For example, when directly asked about the influence of gender on their interactions with 
other professionals, many participants initially expressed confusion or denial that gender affected their work or working relationships, such as when a FRYSC worker commented, "No, I don't perceive that." A sexual abuse treatment center administrator also shared, "I don't see that here so much."

Interestingly, participants who identified themselves within one or more categories of minority status, expressed a greater awareness of the direct influence of gender, and in particular, its intersections with other aspects of their identities. Intersections of gender, race, and rank potentially complicate interpersonal and interprofessional relationships as described by an administrator who shared:

Being a black woman [in human services] is hard. Sometimes you get vibes from people and they're really standoffish..., I try to avoid that elephant in the room. I'm here for something different. Sometimes it's hard for me being a black woman and being a supervisor. I think it might be a combination of me being a woman, me being black, me going from being a co-worker to supervisor, those three things really are the hardest for me. They probably would never admit this, but one person in particular would not accept supervision from me, but would have from [name of a white female colleague].

In addition to the complexities of her professional relationships with co-workers, she also explained how gender differences influenced her interactions with client families. While responding to a question about how women and men do human services differently, she observed:

I don't know if it's so much of they do it differently..., I think maybe sometimes when it comes from a guy..., like if, say if the case manager is a guy then there's a mom that they're working with, she's more apt to listen to him. I don't know if it's like that dominance or because it's a man, I feel like guys have a way of saying something and being a little bit more stern that seems to be more respected than me as a woman if I came in there and told them..., they wouldn't be as receptive. 
Her comment supports prior research that indicates men working in traditionally

feminized care fields tend to exert greater authority and engender greater respect for their work (Bartky 1991). For example, a nurse practitioner, who identified herself as a Black women, shared the following observation:

Human services are geared more towards women. We're expected as women to be more loving, more caring, more nurturing, and that's probably what you expect out of someone who's working in that particular area, but nurse practitioners get paid eighty percent of what the physician rate is. We're considered a lower provider, but we're giving exactly the same care. The state of Kentucky requires us to have a collaboration with a physician and that's for writing prescriptions. Most nurse practitioners pay a physician to be their collaborator. It's required by law. Most of the time it's a paid position if he's willing to be a collaborative, and it's all up to him as the physician. At any point they can decide they don't want to do it anymore. Mine is a male and most physicians are male, and a lot of nurse practitioners are women. If you want to sue me, even though I have a collaborator, he won't be affected at all. It would only affect me.

When perceived and recognized, it appears that men are accorded more respect for their expertise and authority than are women and this issue is compounded when race and higher status or authority intersects with gender, particularly for the Black women who participated in my study.

The complexities of intersectionality were further demonstrated in an exchange between two co-workers following a focus group interview. The women wanted to speak longer after the full focus group concluded about their experiences of gender, race, and safety relative to the regional characteristics of their service area in Kentucky. One of the women shared:

It's hard being a black woman doing this [human services]. It doesn't matter how many degrees I have or what knowledge base I have, I'm still a black person in your house trying to tell you how to raise your kids and we are in Kentucky, and that just doesn't sit well with some people which is why there's some areas I just don't want to go and that's also a reason 
I'm not going to anybody's house out in the middle of the county without a [CPS] worker. I'm just not doing it.

In reply, her colleague expressed a more ambivalent attitude about her own experiences of race, gender, and regional work with families, as well as sympathy for her co-worker's concerns when she commented:

See, I've got a different perspective. I don't care to go [do a home visit] without a worker or whatever. I sympathize for your issues. I don't have them. I'm sorry. I wish people didn't treat you that way. That sucks. I don't think about being white ever to tell you the truth, and I really don't think about being a woman much.

Their exchange exemplifies some of the ways in which complex intersections of privilege may be more apparent and more relevant to individuals who chronically experience multiple sources of disempowerment (Hill Collins 1998; Newman 2007). At the organizational level both women were tasked with doing the exact same human services work because they were hired for the same case management position; however, at the personal, interpersonal, and professional levels, their individual standpoints and experiences diverged in extraordinarily meaningful ways.

\section{Ethics of Care and Ethics of Justice}

Drawing upon Carol Gilligan's theory of ethical decision-making as an explanatory framework for this theme that emerged from the data, I explored the ways that rationalized systems of bureaucratically-structured service collide with a mandate to care for families and children in crisis (Gilligan 1993). Gilligan's discussion of ethics of care demonstrates how systems of care such as those situated within the human services field are profoundly limited by bureaucratic constraints. Following Gilligan and similar scholars (Kagan 1991; Kagan 1993; Martin 2004; Martin 2006; Sowa 2008; Acker 2012; 
Weinberg and Campbell 2014), I maintain that these systems are gendered in ways that are often incompatible with interagency collaboration.

According to Gilligan (1993) and others (Hugman 2014), ethics of care are rooted in gendered constructions, gender socialization, and gender identities, and are situated within hierarchal gendered relationships characterized by differential access to power. To enact an ethic of care is to adopt a more feminine orientation that views people as "embedded in social relations in which all types of attachment (emotional and biological as well as contractual) are regarded as core values that should be nurtured" (Hugman 2014, p. 178). Such a perspective acknowledges that personhood is grounded within social relationships. Gilligan (1993) stated that masculinity is alternatively defined through separation, self-reliance, firmer personal boundaries, independence, and competition.

Human service organizations are social environments wherein ethics of care are regulated by bureaucratic constraints that impose a masculinized structure of formal rules, regulations, policies and procedures aligned more closely with the legal-rational standards of an ethic of justice. The legal-rational structure of masculinized bureaucracy does not parallel, complement, or accentuate the ethics of care embodied within human services work. Thus, a major critique of bureaucratic human service organizations posed in my dissertation is that these resulting tensions may further exacerbate the problems of at-risk families and children as well as intensify the gaps among collaborating organizations. As demonstrated in the composite case study, an integrated spectrum of human services collectively made efforts to protect both Candace and Drew from different types of harm; however, the resulting mandates were bureaucratically imposed 
in ways that neither parents could emotionally or financially cope with or realistically manage.

Ethics of care within human services are enacted through professionalism - the policies, procedures, and practices that are formalized by each human service discipline (Hugman 2014; Weinberg and Campbell 2014). Ethics serve to guide, protect, and empower human service workers and client families and as a way to determine "matters of right and wrong and matters of duty and obligation" (Reamer 1998). Professional ethics are necessary and beneficial, yet, when formalized, standardized, and bureaucratized, the intentions of ethics may become an extension of justice rather than of caring.

\section{Professionalism and Ethics}

Human service organizations are bounded by codes of ethics, sometimes flexible, sometimes formal and rigid; however, professional ethics within human services promote both care and justice. A licensed family therapist described his professional ethics:

I always go back to the five ethics of counseling. Number one is the autonomy that we are to give our clients. The autonomy for self-direction of governing, that's one of the things that I'm a huge advocate for is people's autonomy. The second part of the other four is non- maleficence, beneficence, doing good, doing the good that we can, doing no harm. Another one would be fidelity and [fourth] having the loyalty to my clients. And then the last one would be justice. Those are five ethical components I think are very important, and I hold all of those very, very sacred as a counselor because those are kind of my governing..., those are what my instincts come from.

Similarly, a judicial case manager discussed the expectations that family court judges have for the professionalism and ethics displayed during court proceedings and related collaborative interactions in their work to represent at-risk families and children. She commented: 
There's a high expectation [in family courts]. There's a high expectation of professionalism, there's a high expectation of doing what's right and then more, there's a high expectation of work ethic, there's a high expectation of don't pass the buck. Those all may not be said out loud, but those are the expectations and that's how the judges approach the situation and that's what they convey down.

Her account reveals how deeply and how overtly the fundamental undercurrent of professionalism and ethics is normatively expected within the professional culture and within the organizational cultures of human service systems. Her comment also demonstrates hierarchal systems of authority and accountability that are systemically embedded and tacitly "conveyed” among human service organizations.

Ethics are a core, structural characteristic of professional work within human services (Hugman 2014). Human service organizations rely upon formalized codes of ethics as a way to integrate "caring about" and "caring for" at-risk populations into particular types of professional actions (p. 175). Whether service tasks are specialized and highly skillful or mundane and perfunctory, the ethical traditions of human service professions reflect the broader, and, for the purposes of this study, gendered culture. Previous research has considered the ways in which ethical codes and professionalism have come to focus more on the need for objectivity, distance, technical skills, and attaining expertise rather than on enacting professional expertise as informed, quality care (Held 1993; Kohen 1994; Ruddick 2002; Hugman 2014). Their results bolster my dissertation findings which demonstrate how the bureaucratic structure of multidisciplinary human service organizations perpetuate and reproduce gendered relations of power and authority contextualized through constraints upon ethics of care.

Although the interview questions I asked explored individual motivations, strengths, and barriers to interagency collaboration among human service organizations, 
the responses of a vast majority of the participants emphasized the constraints that bureaucratic systems imposed upon their work, work with intentions embedded within ethics of care. The organizational professionals I interviewed represented a diverse array of human service disciplines. Yet, as different as each of their explicit missions and goals were, my study demonstrated several shared characteristics among the organizations.

First, they were all bureaucratic entities with a hierarchal administrative structure or system of oversight. Second, each of the organizations had an established professional culture, and most had their own formal disciplinary set of ethical guidelines. Third, all of the organizations had a distinct mission and most had established a formal mission statement with related goals that shaped outcomes that were officially tracked. Fourth, and most importantly for this purposes of this study, the ability to establish or maintain professional relationships among the human service organizations was influenced by hierarchies of professional rank and relative levels of power, authority, and professional expertise, a major point that will be explored in greater detail in the last section of Chapter Five. The following organizational example further reveals tensions between ethics of care and ethics of justice and how filtering professional ethics through bureaucratic systems perpetuates hierarchies of power, rank, credentials, and the subordination of client families.

An organizational example - CPS. One prevailing example of a centrallyintegrated human service organization wherein ethics of care were frequently viewed by a majority of research participants as bureaucratically regulated was Child Protective Services (CPS). Participants consistently described CPS in particular as consisting of 
caring individuals, mostly women, and many as social workers, who expressed a strong commitment to and a passion for their work as change agents serving at-risk families and children with dire needs. Yet, participants concurrently acknowledged the bureaucratic limitations imposed upon their caring work and they described the resulting outcomes as punitive and disempowering. More detailed descriptions of bureaucratic constraints will be presented in the next findings section.

As a government operated organization within every state, CPS is tasked with investigating reported allegations of child abuse and neglect and making informed judgments about the substantiation or non-substantiation of those claims (CHFS 2016). Federal legislative guidelines, primarily the Adoption and Safe Families Act of 1997 (ASFA), require CPS to focus on three overarching organizational goals - safety, permanency, and well-being (CWLA 2016). What this means is that however caring CPS staff may personally be, the boundaries of their profession — ethical, caring work — is strictly regulated by rigorously detailed legislative statutes translated into vast numbers of formal bureaucratic policies, procedures, manuals, and forms.

Fifteen of the 51 participants I interviewed, approximately 29.4 percent, were either currently working for CPS, were contract agents providing services solely for CPS client families, or had at some point in their professional careers worked in a CPS role. Their years of employment in a CPS capacity ranged from two years to nearly twenty years. All fifteen participants had experience in both urban and rural communities. Five of the fifteen participants had worked on both investigative and ongoing teams. One of the fiftenn participants had only worked on an ongoing team. To clarify the difference, an investigative team of CPS workers conducts formal investigations of child 
maltreatment referrals that meet eligibility criteria based upon the allegation information provided at the time of the child maltreatment report, such as the professional represented as Bill in the composite case study. Ongoing CPS teams, such as Debra in Candace's story, provide regular and ongoing case management services to families for whom one or more reported allegation of child maltreatment was investigated and substantiated, or found to have occurred based upon supporting evidence.

One human services worker described his experience of beginning his professional work in a rural CPS office. He completed a social work degree because of his desire to "help people in need," but very quickly his CPS role became overwhelming, isolating, constrictive, and constrained by competing bureaucratic priorities and a lack of access to necessary community resources. As a "general" CPS worker in a rural county, he described how his caseload was made up of all types of CPS cases from investigations, to ongoing, to foster care case management, and to residential oversight, "everything all at once." After several years of working in a rural CPS environment, he moved to a more urban CPS office wherein the focus became investigative casework. While describing his experiences of working in both settings, he commented:

I'm in the middle. I'm like stressed out, working on Saturdays off the clock, my blood pressure is up, I gained like 80 pounds. I can do all that stuff, thinking like, 'How can I make something happen on a bigger level. I need to do something else. This is going to kill me.' Or, you can leave, or you can be so crisp and burned out that you don't even respond to emails. The nature of the Cabinet is that unless you cheat on your time sheet or your travel, you ain't getting fired, and that's just the truth. So, like you can just be..., they call it performance punishment, that's what it's called. Your reward for working honestly and ethically is more work or you get all the referrals from judges and law enforcement which is like six [referrals] in one. So, yeah, it's one of those things. It's awful. 
This social worker's account illustrates how bureaucratically-structured human service environments can lead some employees to feel burned out and unmotivated to do the work of the organization - the critical, often life-saving work of serving at-risk families and children. He, like many other participants I interviewed both formally and informally, described how bureaucratic stressors within his previous organization led some workers to shift their concerns away from a focus on caring and to prioritize concerns about professional accountability and liability instead. The worker described above continued:

The ethics of day-to-day practice were underneath concerns about liability just given the bureaucracy and the whole nature of it... That's why people leave. They're gone. They burn out... You realize that it's an effective strategy to not do quality work. You realize that. The way that the system is they're [families] at the very bottom.

According to his experiences, prioritizing concerns about professional liability, meaning the risk of personal and organizational litigation in the event of practitioner negligence or malpractice, were not only be at odds with ethics of care, but also displaced professional investment in exerting care through human services work, especially within the CPS and similar human service contexts (Reamer 2001). This CPS worker explained:

How can we find individuals who are willing to say, 'What's right is right, and I'm just going to do what I have to do to protect a child?' Or, to stand up and say, 'I disagree, Your Honor. This is what I've seen,' because often, through my whole experience I feel like the child welfare workforce, some of them that are trying to do their best are isolated and alienated, and they' re like trying to keep their head above water... The point is to say something through your professional experience that can be grounded in practice to make things better for this family, ... but the end result is you have a few people that are probably burned out that are doing the same dag gone thing every day, and they're probably not willing or not able or whatever you want to say to stand up and make a stand and say, 'Look, it is what it is. I don't care. We're going to put this in writing because the child deserves better than this. 
The functions, roles, ethics, and concerns about liability are included in the core curricula for formal training of new human service workers and ongoing professional development for existing workers (Hugman 2014; Weinberg and Campbell 2014). Through formal training human service workers are socialized and immersed in the professional culture of their organization. In keeping with the example of child protective services, another CPS worker who assisted in a consultative and training role, shared her experiences related to the conflict between caring and "the work" of new CPS front-line staff. She described what new social workers learn from the formal training curricula versus the training they receive "in the field," especially in balancing personal and professional responses to the work and to other human service disciplines. She stated, "We try to engage with our new workers and letting them realize it's a job, and everyone has their role, and not to be offended when someone is doing their job." Discussing how workers are trained to adopt ethics of care entrenched in professional objectivity with her helped me to realize her deep level of care for the emotional wellbeing of new workers, mostly young adults in their early twenties and mostly women. Yet, her concerns were also for retention within an organization that experiences high turnover because of the immensely stressful nature of CPS work, which has been estimated to be as high as 40 percent within the first two years for new CPS front-line employees (Yankeelov, Barbee, Sullivan, and Antle 2009).

In academic and professional training social workers are taught to keep a professional distance from clients in order to safeguard their own wellbeing, the wellbeing of the family, and to avoid personal bias as much as possible. While at the micro-level acquiring an ethic of objectivity may serve a protective function for caring 
human service workers, the same ethic, when institutionalized and bureaucratized, may take on a more rigid, formalized quality of depersonalization through policies and procedures that stipulate and enforce ethics of organizational objectivity. Workers trying to maintain an appropriate professional distance follow guidelines, policies, and laws often find their professional discretion mired in a web of rationality. For example, another human services worker who had resigned from CPS after two years of working on an investigative unit, shared his experiences. He described the rigorous training he had received during the first six months, but his experience quickly changed once he became an active CPS worker. While discussing his perception of his prior investigative role with CPS, which in Kentucky is a branch of the state's Department of Community Based Services (DCBS), he commented:

Unfortunately, DCBS on paper, it seems like, you know, one of the most conventional forms of social work, but, in reality, there's a lot of political elements to it, a lot of red tape everywhere you go within the agency, and, that disconnect from the agency to the individuals we're providing services for almost seems to be an essential part of your tool kit for any kind of longevity. You have to separate yourself, your emotions, from these people, and provide services, and, it is my opinion that, as social workers, inherently we're there to help remedy social issues..., we're on the front lines for remedying social issues. I didn't find that to be the case. I found DCBS, even ongoing, but, more specifically investigations, what I did, to be more of an extension of law enforcement.

Comparing CPS work to the role of law enforcement in the above quote demonstrates another way in which ethics of care may be transformed into an ethic of justice, an ethic which either intentionally or unintentionally promotes a retaliatory or punitive approach to serving at-risk families with needs. Workers expressed pressure to either clear cases or keep cases longer in order to avoid new referrals, extraordinarily high caseloads, and 
competition to avoid accepting new cases or to avoid getting the most intensive types of

cases. For example, a social work field trainer shared:

We get very caught up in turf, us versus them. Instead of stepping back and using those social work skills, step back and assess situations, think about where the person is coming from because if I'm providing services, I need to meet clients where they are. That's part of social work, and I think that as professionals we forget to do that with one another. I think we are overworked, overburdened, underpaid, stressed, trying to fit in our own stuff. We got into this because we care about others, we care about making a difference for at-risk, vulnerable populations. All these other pressures of things, we forget to treat each other as professionals with the respect and courtesy that we're trained to do, that we're supposed to show to our clients. That starts with us. I don't think it's intentional most of the time, but it happens because of too many cases, too many deadlines, and in child protective services, being so focused on deadlines and past dues that we fail to take into consideration that you and I both have 30 cases, but 30 cases can look totally different for you versus for me. If you have five that are sexual abuse or near death or I have 30 cases where half of mine have four to six children and yours have one to three? It's overwhelming.

This social work field trainer's account further clarifies the immense complexities of case work amidst bureaucratic constraints and limitations. Social workers who feel overwhelmed may experience disconnection from their core skill set and inhibited from collaborative support.

Another licensed social worker involved with social work college students described a common response from advanced field placement practicum students who had opportunities to observe real-life professional social workers "on the job" in the following way:

Students are seeing collaboration that does and doesn't work well, and I've dealt with that, too, with the student calling me and going, 'You know, this has not been a good experience to see professionals interacting together.' Well..., welcome to professional work. It's an eye-opener. 
The students' comments illustrate how even untrained and unseasoned beginning workers can see when collaboration is not working as well as it could, or as well as they have been educated to believe that it should.

The training to become a social worker further exemplifies the focus on regulated mandates and formalized procedures as demonstrated in the following description provided by the same licensed social worker involved in coordinating and overseeing field placements for social work students at a public university. First, she described aspects of the training for social work practicum students:

Not to get too much alphabet soup, but we tend to like those things in the professional life. So CSWE, which is the Council on Social Work Education, accredits social work programs, undergraduate and graduate programs across the United States, and our program at the bachelor's and the master's level both are CSWE accredited programs. In 2008 our accrediting body, CSWE, went to a competency based standard, meaning that they came out with ten specific competencies and 41 practice behaviors under those competencies or within those competencies that all social work students at the graduate and undergraduate level should have and attain successfully before they can graduate and be a professional social worker.

Next, the social worker described the disjuncture between how social workers are educated through formal curricula versus the lived realities of the ongoing training they get in the field. She observed:

You have agencies that have policies that don't meet the [social work] code of ethics. I deal with that when talking to students and talking to agencies about it. Let's say that you're a social worker and our code of ethics say it's the client's right to self-determination. Let's say you go to work for a pregnancy support clinic that is very faith-based and doesn't believe in abortion. There's a conflict with your professional code of ethics and the agency you're going to work for. Can you work there? 
The preceding excerpts show how masculinized bureaucracy and bureaucratic constraints are threaded throughout the experiences of becoming a human service professional in ways that inhibit ethics of care.

\section{Additional Bureaucratic Constraints}

Additional barriers to interagency collaboration described by participants included time constraints; budget and funding constraints; service overlap and turf issues; a lack of reciprocity; and numerous types of communication barriers. Similar barriers have been acknowledged in previous studies; however, each type of barrier was also richly described by the participants. Almost every participant described the intense challenge of time management and how being busy was a concrete obstacle to interagency

collaboration. An in-home services worker shared her experience with time management during a focus group interview in the following way:

Having a good working relationship is hard to get when you end up with a referral from somebody who doesn't answer the phone from the very beginning..., you know what I mean? So how do you really make..., and, then also, they're busy and we're busy, too, you know, so we also, other than the timeframe, have other cases going on and things where we don't necessarily have free or extra time to, I don't know, to track somebody down for collaboration.

Similarly, a mental health service coordinator shared her concerns about how unrealistic finding sufficient time could be for effective collaboration:

One of the biggest problems, and I'm going to tell you right now, I love it if I can invite somebody else other than the parent, the therapist, and myself to a meeting because that means that these people are vested enough in this child's future, and, they want to see this child meet their goals. They care enough that they're going to be there, but a lot of times it's not realistic. Social services, I mean, it's hard just to get them on the phone much less to get them here because they're busy. You know? They've got such a hectic schedule. So, sometimes we just have to make those phone calls and that has to suffice. 
Her comment demonstrates how human service workers are forced to accept whatever level of accessibility, availability, and contact they can get from other collaborating organizations.

Adding to concerns about insufficient time and worker availability, high caseloads and competition were mentioned as frequent barriers to interagency collaboration, especially within the area of foster care placements. An agency director acknowledged these strategic barriers when he described how there is intense competition between private foster care agencies who sometimes are "bidding" against each other for the same children who are in need of a temporary foster home. Not only do they urgently compete for the same population of high need, high risk clients, they also compete for a pool of skilled professional staff. He shared:

Competition creates these silos and these walls. When we did job interviews, we interviewed three people from other agencies and each of them were carrying well over the maximum case load. One said 26. She was carrying 26 cases when the maximum per state law for everyone [doing foster care] is 13 .

His comment demonstrates how many bureaucratic constraints combine to produce work environments wherein providing a higher quality of focused, responsive human services is extraordinarily difficult. It also reveals how competition ratchets up the tension between organizations that provide similar types of human services making it more difficult for them to collaborate in serving the specialized needs of vulnerable families and their children. A foster care placement coordinator expressed similar concerns when she discussed her experience of attending statewide meetings wherein competitors were present and were openly guarded about collaborating. She commented:

A lot of providers never talk to each other. It's funny. I went to a supervisor's training and we had to do group work, and, of course, I was 
the only one there from my agency. There were lots of other people. He kept saying, the trainer, you know, he said, "You guys aren't saying a whole lot," and someone said, "Well, we don't really want to share our secrets because the possible competitors in the future are in the room." I think there's a lot of that that kind of hinders collaboration, and I don't know if other agencies have policies where they don't want people talking to other agencies for probably multiple reasons, but I think if you're in this field, if you're going to do a good job, there's got to be collaboration. Whatever we can do to help that client succeed.

When organizations are guarded because of concerns about maintaining sufficient staff retention, clients, or funding, interagency collaboration cannot work well. More concerning is the idea that the needs of vulnerable clients, and, in the case of foster care placement serving traumatized children, may either inadvertently or intentionally become secondary to bureaucratic priorities. The coordinator's experience further reveals that collaboration (or the lack thereof) is not solely a localized concern. She attended a statewide meeting of providers; therefore, the potential for competition to disrupt interagency collaboration is broad scale and pervasive.

While describing similar concerns about the tensions between competition and collaboration, a licensed social worker discussed a prior experience with providing local parenting education classes. She elaborated by sharing the following account:

When I was doing parenting classes, before I did parenting classes I read through the curriculum at the agency, but I hadn't ever taught a parenting class before. I remember calling a local agency in town to ask them, "How do you all provide parenting classes? We're going to be doing that. Do you mind if sit in?" There was dead silence on the phone when I said that. It was not okay, and they were not interested in having another nonprofit in the community that was going to be providing services similar to what they do sitting in on how they do them. To me, and that's something that has stuck in my mind years later, it speaks to collaboration. Would that not cut down on the waiting list? Not everybody looks at it the same. 
Her comment reveals how competition for scare resources could serve as a major barrier to open communication, collaboration, and serving the best interests of vulnerable families and children.

The nature of very serious and chronic safety concerns for families and children amplifies the heightened intensity of crisis care work. Families who come to the attention of human services commonly experience multiple concurrent problems, any one of which could be a reason for the involvement of numerous human service organizations and programs (Brown, Cohen, Johnson, and Salzinger 1998; Sprang, Clark, and Bass 2005). The multi-problem dynamics of at-risk client families regularly result in or raise concerns for child maltreatment, especially the risk of poly-abuse, meaning a risk for multiple, concurrent, or recurrent incidents of child abuse and/or neglect (Gelles and Cornell 1990). The intensive, often imminent nature of multiple needs is a prevailing rationale for multiple services being provided as an integrated form of "wraparound" care (VanDenBerg and Grealish 1996). A mental health services coordinator described one of her recent collaborations in the following way:

Let me give you an example. You have a family that needs an interpreter who doesn't speak English. You need an interpreter. Okay, you need to have a service team meeting for them. You have to collaborate. You need to make sure you have the interpreter there, anybody else that's on the team whether it be like, [a rape crisis center], like [Brenna* a well known local therapist], like what she does, you would like to invite them, child protective services, you'd like to invite them, the therapist, and, hey, the most important person is the family. So, you have to make sure that everybody can attend that meeting. So, you're making phone calls, you're saying, "Hey, can you be here?", "Can you be here?", "Can you be here?", and, [if so] that's wonderful. Now, let's say you only have the interpreter there and you just have the family and it's just you. Alright, you're not gonna give the input from the teacher, "Little Johnny's having problems in school." I mean, I can go to the school, but we would prefer for them to be at the service team meeting with us, right? So we really try to collaborate all those services. Okay? And, during that meeting, we talk 
about our goals and stuff like that, and, part of that service team meeting and that service plan we talk about, "Hey, what's your job gonna be?", "What's your role gonna be?", "What's your role gonna be?" Who's going to do what? So that we are making sure that we are truly doing that wraparound philosophy.

Interagency collaboration is time consuming to coordinate and takes a lot of effort and planning as illustrated by the coordinator's excerpt. She speaks to both the need for collaboration, especially when a client family has multiple intensive needs, but also the difficulty in allocating sufficient time and resources for all the "players" to meet together in the one place at the same time. Interagency collaboration is a foundational principle embedded within the frameworks of wraparound models (Burns, Schoenwald, Burchard, Faw, and Santos 2000; Mattessich, Murray-Close, and Monsey 2001; Warmington, Daniels, Edwards, Brown, Leadbetter, Martin, and Middleton 2004); therefore, barriers to collaboration have the potential to broadly disrupt and fragment the nodes of integration within a systemic human service organizational network.

Drawing on Marx, this concern is further informed by the work of Bartky (1990) who said, "The capitalist organization of production is that workers lose control of what they have produced; their products cease to be mirrors in which they are able both to affirm and to enlarge their distinctively human capacities; these products serve instead to enrich the capitalist and to augment the power of capital, an 'alien force' inimical to the worker's vital interests" (p. 34). Unlike Marx, who argued that capitalism was at the root of such alienation, in human services bureaucratic constraints reproduce a culture of domination and alienation, perpetuate hegemonic masculinity, turn caring work into drudgery, and create then maintain many of the barriers within collaboration that efforts to collaborate attempt to confront and challenge. Within human services, legal-rational 
bureaucratically-structured organizations also work to efficiently produce changes in terms of the outcomes for human lives. Thus, both human service workers and the families and children who receive services may struggle to conform to bureaucratic rather than caring organizational standards. In the next section I further illustrate how power, as a predominant bureaucratic constraint, is institutionalized, gendered, and intersectional in ways that are relative, subjective, and exclusionary.

\section{POWER AND INTERAGENCY COLLABORATION}

The human service workers I observed and interviewed for this study consistently mentioned a desire to help others, to "remedy social issues," and "to make things better" for families. Hearing them talk about their ethics of care resonated for me. Their stories helped me to remember the many colleagues I have worked with throughout my professional career, all the women and men who I consider to be dedicated colleagues and genuinely caring people. The experiences that the study participants shared also illustrated another side of human services, though—a side voiced with strong consensus. They voiced their frustrations, sometimes to the point of burn out, with the rational constraints of bureaucratic systems they felt regularly encumbered their caring work with and for at-risk families and children. As stated previously, bureaucratic constraints are a form of power, but they are not isolated from other everyday cultural practices of human service professions (Rossiter 2000). Power is embedded throughout human services work, work which "establish[es] as normal and natural what has, in fact, been constructed through complex power relations" (p. 150-151).

Power may be exercised legitimately through organizational authority; however, power may also be exercised in interactional and discursive ways (Foucault 1979; West 
and Zimmerman 1987; Bartky 1991; Rossiter 2000). Thus, the hegemonic assumptions of human service workers, in both overt and subtle ways and sometimes in unintentional ways, are reflected in their professional practices with other professionals and with client families who are sometimes silenced. These tensions between bureaucracy and ethics of care result from masculine, bureaucratic exertions of power within dynamic, interactive, intersectional complexities of integrated human service systems. There are three overarching implications of the tensions:

1. power is relative;

2. power is subjective; and,

3. power is exclusionary.

Focus group and in-depth interview participants openly acknowledged these disparities, and I also witnessed them during participant observations. In the following sub-sections, I present an in-depth critical analysis of relative, subjective, and exclusionary forms of power within human services.

\section{Relative Power}

Power is relative to hierarchy, rank, and authority among human service professionals, and relative to being a human service professional or a client family. This is not to say that families never exert power over their own decision-making or outcomes. Rather, according to the participants' experiences, they viewed a majority of the client families with whom they worked as chronically disempowered by many of the same bureaucratic constraints to which they were subjected and with which they regularly felt frustrated and inhibited. The relativity of power among human service professionals will be discussed first. 
Relativity of power among human service professionals. The first account is

given by an unlicensed human service worker during a focus group interview who

described how case managers are frustratingly ranked on a bottom rung of power, a

location similar to the families being served:

It's power..., it's a dictator's kind of thing. There's a totem pole and you rank somewhere on that totem pole. We're [case managers] at the bottom. Court goes at the top because they make the actual decision. Court, DCBS [Department for Community Based Services, another name for CPS], therapy, schools, then there's us. I really kind of feel like that's how that rolls, and the family is at the mercy of everyone else. They've got the hoops to jump through, they've got to put the work in, and we've got to fight our way to the top. We've got to build those relationships as we climb this totem pole. We've got to hold that family's hand and we've got, together, we've got to climb this totem pole and make friends with all these people that supposedly have that higher power.

Conversely, a licensed therapist described the need for hierarchies of power and ranking within human services when he commented:

Sometimes power struggles are necessary. Sometimes they're needed. Sometimes..., because of ranking..., because I do feel like if you're a licensed professional in the state of Kentucky you should pull rank. If you're not licensed you need to get out of the way. It doesn't mean your information isn't valid or important. It is. I feel like licensed professionals have a little bit more weight in the game..., more skin in the game. And that's another part of collaboration. I am worried that people who hold the title of case manager or case coordinator or whatever..., like, there really should be a license for those people, and there should be ethical codes and guidelines for those types of people who work in that situation because I think it's becoming a profession. I mean, it is a profession that's out there. I feel like they're untethered, and could use some support.

Both participants' concerns expressed an interest in supporting client families' needs based upon ethical goals; however, the ways that power was embedded in ranking and authority was a precarious barrier for each of them. A licensed social worker who had previously worked for CPS similarly shared: 
There's also a hierarchy beyond the client. Maybe the client is there or the client's not, so if I'm just throwing out speculation based on my experience you've got people like law enforcement which are basically swinging the biggest bat. We're all sitting there at the GUS [Growing Up Safe] meeting and what they say goes. So the fact of the matter is there may be criminal charges. They have different goals than we do. We're trying to keep families together and provide for safety and they're trying to see if people broke the law, and, if they do, they're going to respond accordingly. So there's that, and unfortunately I've inherited collaborations where you've got..., you know, my goal, like you asked me at the beginning, my goal. Will it be the same mission? The same goal? There are differences.

The following excerpts demonstrate a range of bureaucratic constraints which are gendered, institutional, and intersectional in origin and orientation. The predominant barrier was power. Power was described or implicated as a barrier in several different ways. Power was expressed through professional language, the use of specialized argot or jargon and dress as well as confidence in one's professional presentation as was illustrated by a law enforcement officer when he commented:

We depend on these troopers to be a whole lot of things. Most of that academy up there is teaching you how to not get killed. That authority, that position of authority, that hat, the uniform, all those things, it's teaching those guys when they get out there, when you step out of that car, you have to be in charge. Many times it's not a pleasant experience and you have to be the one to take charge. That arrest warrant doesn't say if it's a clear day and you don't have anything else to do take this guy to jail, or if he really wants to go, take him to jail. It says you are commanded by the Commonwealth to take this person into custody. It's not really a choice you get to make.

Power was embodied through decision-making influence and legal authority. It was expressed through organizational sense-making and the ways in which client families, their "issues," and their needs were categorized and documented through textual and verbal acts (Weick et al. 2005). For example, a judicial case manager described the 
necessity and the importance of discourse and documentation for family court

proceedings. She commented:

Some agencies aren't comfortable either appearing in court or providing written feedback, and that is essential for a court case. We can't just say, "Well, the therapist told the mom this, this, and this. I mean, there's going to be an objection really quick. So, agencies who don't want to, because of the legal hurdles, or who fear court involvement, it's really hard to rely on them.

Her description demonstrates how courts rely upon agency information for critical decision-making on behalf of client families, and she went on to describe how human service organizations and professionals may be subject to court orders and subpoenas as measures to ensure that information deemed as necessary is received. Thus, power was embedded in the hierarchical rank order of different human service disciplines and organizational affiliation. Accordingly, an in-home service worker commented:

We were usually asked to go or subpoenaed to go to court, and sometimes they didn't ask our opinion and I wished they would so I could share, and it could be either way, either advocate for the family, or, wow, they're really missing information. Sometimes I wish in that aspect I had been heard more. I don't think it would have been too appropriate to raise my hand in court. When they make their ruling, that's the judge and I'm not going to question what they have to say. They have our case notes, they have our information, so they read over it, and they pick what they want from it, so I don't feel like it's my standing to question what their ruling is. That's their job, because sometimes because I work closer with the family, I can be opinionated. That's just natural in this field.

Her experience of going to court and being involved in the judicial process illustrates relative power on a number of levels. First, judges preside over human service workers and the family in child maltreatment cases; therefore, professionals may or may not be asked to fill in or flesh out the details of their notes or recommendations unless judges specifically ask them to do so. This may be a gap in collaboration. Secondly, the excerpt 
demonstrates a tacit awareness of relative power and authority that is accepted as normal and natural to doing the work of human services. Third, and lastly, her notes reveals how the legal process makes sense to her and other human service professionals, and there are clear processes, procedures, and rules based on how professionals and client families are oriented, categorized, and discursively engaged through textual and verbal acts.

Power was also evident in response to discourses about professional liability and fear of reprisal for breaching organizational policies or for a failure to in some way safeguard at-risk families or protect vulnerable children. Ironically, these same fears were viewed as inhibitions for doing "the right thing." An agency director described his perception of this form of power as follows:

I think most people are here to help someone, and I think most of those people who are here to help know that collaborating from agency to agency makes the most sense, but everyone is too damn afraid to do it, and that's why they embrace the idea of it, but, more often than not, we don't get there. Now we make a whole lot of phone calls to people and they say, 'You didn't hear this from me.' That gets into all kinds of legal issues which we shouldn't have to get into. We should just be able to collaborate with one another.

His comment speaks to the inhibitions professionals often experience when they are asked to openly share information about shared client families during interagency collaborations. In essence, he is saying that sometimes confidential information is not shared when it can be and, at other times, it may be shared when it should not be. Either way, collaboration may be affected by the relative power of professionals and through the ways in which they negotiate an exchange of client knowledge. Relative power influences relationships among professionals, but, more importantly, relative power also shapes social interaction between professionals and the client families to whom they provide human services. 
Relativity of power between professionals and client families. Professional culture and codes of ethics are necessary social structures within human services; however, previous scholars claim that such standards create and sustain a one-sided and top-down hierarchy of power between professionals and client families (Cornell 1992; Orlie 1997; Weinberg and Campbell 2014). "We must also recognize the structural constraints and paradoxes that are part of the everyday experience of practitioners while we conceptualize practice as a political activity that either reinforces inequities in society or moves toward social transformation" (Weinberg and Campbell 2014, p. 48). Human services then is far more complex than simply helping or having the desire to enact ethics of care. Human services consists of intersecting, interpersonal localities situated within dynamic relations of power, including relative power.

So, while power was sometimes exerted in authoritative, coercive, and reportedly necessary ways among human service organizations and professionals, participants described clear and definitive power differentials within interactions with client families who understood the consequences of failure to do as they were told and to submit to worker's recommendations or demands. An administrative social worker, commented:

People tell us what they think we want to hear because they're scared of us. We need to emphasize to them because of the role and what we represent, even though we can't technically remove a child ourselves, we can petition the court to remove the child, and people are scared of that authority.

Her comment acknowledges that client families respond submissively and fearfully to human service workers, in particular CPS workers who are perceived as exerting more authority. She further acknowledged that CPS does indeed have power and the willingness to exert it if child safety is in subjectively questioned. In addition, an in- 
home services worker shared a similar acknowledgement of the relative power in-home case managers have in relation to recipients of their services:

We get really comfortable and we think our way is best or sometimes we just don't want to find another way. Then there's always the whole, 'Well, you're obviously not doing it right because if you were doing it right you wouldn't be having these problems.' We really have to step outside the box of what, not what's best for us but what's best for your family.

Power was enacted by human service workers and was described by participants as being relative to the subordinate position of client family. In another example, a licensed social worker with CPS experience described the ways that CPS workers are trained to exercise power, and how power was used to ethically facilitate client change, but also how power could also be unethically exerted by some workers to coerce client outcomes. He explained:

So you have like no understanding of what you're actually going to be doing at all, and through six months of training they talked about how it takes two years to get like $80 \%$ of the job understood. Like it takes two years to understand cause there's just like unreal amounts of stuff you have to be able to function with. So, it was discussed about the perception of true and perceived power that the worker has. It didn't take long for me to realize that what the worker says goes, especially if you're doing it right and you're very documented, very organized like I was. I never lost a court hearing ever in six or seven years, but it was rooted in policy and practice and it was just what it was. I've heard of other workers in other counties that would do unethical things. Things that were above and beyond policy. They would get into relationships with clients and do all these other things that were totally unprofessional, but the power is real, and the perception of power further magnifies that.

His description speaks to several ways in which professional interactions with client families are entrenched in power. First, training attempts to prepare new human service workers for the myriad ways in which they will exercise power through their organizational roles. Second, power is relatively enacted through the inundation of 
organizational knowledge including learning legislation, manuals, policies, practices, and procedures that bureaucratically governs care work. Third, power shapes both the legitimate and the unethical ways in which involuntary client families are compelled to adhere to subjective standards of normal family functioning.

Another account from a community coordinator of a faith-based clothing donation service for women demonstrates how power is also reflected in the ways professionals prefer for clients, in particular mothers who are recipients of human services, to mimic professional behavior and dress, especially when preparing for court or for job interviews. The coordinator commented:

If you feel like you do not have the right clothes or the clothes don't fit, you don't feel like going anywhere, and it's just like..., it's your selfesteem and you do not try new things that other people would do. But I think they [women clients] come in here..., I think especially the women now think, 'I need to look presentable'. The chances are you walk in to do an interview and they're looking at you, and, I mean, they do. They judge, 'Well she doesn't take care of herself or she is..., [wearing] these jeans. This person's going to represent me at my company or at this restaurant and they come in looking like that, or they come in with a short skirt showing their skin or a too tight shirt, and can I say boobs hanging out, and that's just warning signs for an employer.

Her account reveals how there are relative hierarchies established within the intersecting systems that make up the spectrum of human services, and that power is ubiquitous throughout these dynamically interactive relationships. The relativity of power may be subjective in many ways based upon where professionals and client families fall along the hierarchal spectrum of human services; thus, power is also a subjective experience.

\section{Subjective Power}

According to Heron, ““'The constitution of individual subjectivity through discourses is part of a wider network of power relations in which persons are being 
positioned at any given point, and these discourses may contradict one another.

Subjectivity is, thus, unavoidably multiple and contradictory" (Heron 2007, p. 347).

Power, then, is subjective in a number of different senses. To begin with, participants

were more or less aware of the relativity of their power, and more or less willing to share ways in which they directly exerted power within their relationships with other professionals and with client families. Additionally, the degree to which subjective professional behavior is bound by laws, policies, ethics, or personal biases is relative to uncertainty. Human behavior is unpredictable, especially within the context of individual and family crisis; therefore, as expressed by Lorber (1994), "Personal discretion and liability are particularly necessary for those in positions of authority because of the uncertainties they face" (p. 230).

It depends on which social worker you get, because some of the social workers I think really do take the family's thoughts and feelings and whatever into consideration, but some of them don't. Part of it is social worker disposition. You get more resistance if someone's telling you what to do, that's anybody. It doesn't matter what position you're in, there's a way to tell somebody. It's how you frame it.

In spite of the many bureaucratic constraints and barriers many participants continued to feel persistently motivated to collaborate with other human service organizations. Persistence appeared to be based upon participants' subjective discretion. Some participants expressed strong motivation and were invested in engaging other human service organizations in interagency collaboration. A mental health services coordinator embodied this motivated attitude when she shared:

I'm pretty passionate about working with other agencies just because we know, you know, as an agency by themselves it's really hard to meet all the needs of the client or our population for that matter. Everyone has different needs, and I think every member within the community has a 
different strength. Together as a team we try to meet the needs of our population, our at-risk population.

A FRYSC worker expressed her motivations to collaborate and help families so strongly that she felt comfortable breaking the school's rules. She shared:

My principal will tell you that I'm very much a rule breaker. They know I always do it for the best interest of the kids, but, yeah..., I'm a rule breaker. It [other agencies following their policies] will aggravate me, but I understand it, but, yeah.... Like, one of our policies is you're not supposed to transport. If I have a kid with a 102 fever and their parent doesn't have transportation and they live three blocks away, I'm just going to take them home. Just let me take them home. Other staff will not, they are rule followers, so we balance each other out.

I have shown so far that power is both relative and subjective, and these two qualities of power both foster and reinforce each other. The relativity and subjectivity of power within human services also promote a third suggested implication — power is in many ways exclusionary, but primarily in ways that preclude more collaborative involvement of client families in their own decision-making.

\section{Exclusionary Power}

According to Foucault (1979) an organizational field such as human services exemplifies ways in which professional occupations both classify and categorize groups of people as well creates systems of exclusion. Even though caring fields are constructed out of a culture that professionalizes helping intentions “...there are no easy distinctions between social control and social justice, [therefore] we are left only with the possibility of maintaining vigilance (i.e., doubt) over the social implications of what we do" (Rossiter 2000, p. 152). One profoundly concerning implication of the work of multidisciplinary human service systems is the potential for the exclusion of families from influencing their own outcomes, outcomes that directly impact their lives and life 
chances, outcomes that further shape stability, safety, and intactness. This implication was articulated by a significant proportion of the participants. Their concerns were vital acknowledgements of exclusionary power, but were also indicators of the powerlessness they expressed to change the systems of which they were part as well as the powerlessness situated within case work as experienced by the families. Participants of a focus group interview discussed their concerns about the exclusion of families by stressing how family members' statements may be doubted by other professionals and not taken at their word as legitimate sources of information about their own behaviors. One participant shared:

Families are like, low man on the totem pole. They're below us [as case managers]. Everything they say is taken with, like, 15 million grains of salt. I had a mom who said, "Oh yeah, we're doing really good. We've improved on this," and then the social worker looked at me and was like, "Yeah. She really is. She demonstrates this, and this, and this..." And, like, I'm not going to take your [the mother's] word for it. Let Theresa* who's been watching you [tell me], and that speaks to no standard [for how workers treat families].

Her comment suggests relative powerless on the part of case managers, but, more particularly, the powerful exclusion of family members from legitimately voicing their own issues as well as their own progress towards resolution. Another example demonstrates how language and other forms of discourse within normative professional culture further excludes client families in intersectional ways. An excerpt from my participant observation field notes describes the exclusion of interactional and discursive power:

We kept talking to the family trying to help pass the time in the waiting room. Their mental health appointment had been scheduled for 10:30 a.m. and the time was nearly noon. The lobby was full of waiting clients, some patient, some impatient, some holding young children and crying babies, others trying to temper the mouths of feisty teens, much like the mother 
we were with, but the worker I observed continued to talk to the family openly about their case goals and ongoing service needs. Although others sitting nearby could hear if they wanted to listen, the worker focused intently on her conversation with the family as though they were the only people there. The family was called back to the see the doctor for a behavioral consult and medication management. Finally, it's 12:43 p.m. and my own impatience had been nearly excruciating. The doctor was weary of our professional presence with the family, but, after quick verifications of our identities, he hastily closed the door to a small and now overcrowded office. Referring to their file on the computer monitor he asked questions about Jimmy's* physical and behavioral responses to the previous medication changes all the while with his back to the family while typing their responses into the text fields on the screen. "How's the methylphenindate and guanfacine working out for him?" He looks to us, me and the case manager, first for answers and our silence prompts him to turn impatiently and face the mother and her son. She looks confused and he uses the more common prescription names of the medicines instead. She answers him quickly and uncertainly, clearly aware of his sense of urgency, and I'm not sure if what's she's saying is accurate because she looks lost and the doctor looks confused.... We were in and out in 15 minutes.

The family experienced multiple forms of exclusionary power through interactions and discourse with the mental health facility staff and psychiatrist. Their wait of over two hours among a waiting room full of similarly waiting individuals and families excluded them from an equality of time and availability, especially in comparison to the abbreviated office visit of 15 minutes once they were seen by the psychiatrist. Their lack of expert medical knowledge, too, was an exclusionary disparity as well as a direct barrier to communication while interacting with the psychiatrist. In addition, the family was excluded from being the first and best source of information about their own experience of a change in medication regimen. Lastly, and most important, the family was excluded from mutually respectful, face-to-face, interpersonal social interaction because the psychiatrist was more focused on documenting the contact with the family rather than engaging in direct communication with the mother and her son. 
Another focus group participant clarified other ways in which families are

excluded from discourses about their problems and choices about potential collaborative

resources as well as his impression of how families are sometimes judged and

disregarded by professionals involved in their cases. He said:

A lot of times no one's actually listening to them [the families]. Sitting down with them and letting them tell their story and not placing a judgment on it which is all they've been getting. The judge makes a decision. DCBS makes a decision. Other court people, other case managers are just like, you need to do this or you did this wrong. No one's actually just listening or not saying "You're wrong. You're a bad person." They feel judged. It's kind of sad. If they felt [involved] in that collaboration, I think results could be better because then someone's working with them, not against them. I think that's how families see it, like, "They're working against us. They want to take the kids away. They want to break up our family," not as, "They're trying to help us stay together."

His empathy for the family was notable and expressed a resonating concern for the powerlessness and exclusion of families who may feel discriminated against and unsupported because of perceptions about their inability to resolve their own crisis situations or family dysfunctions. A sexual abuse treatment center administrator mirrored similar concerns when she described the exclusion of clients and families. She commented:

I think they [families] are left out a lot. I know from our standpoint that has to do with confidentiality and accessibility to clients. So, I think they may be left out in that realm. There's a lot of discussion about the families and the clients, and I think we are making some of those decisions maybe without them when we shouldn't be. We really need the clients or users of services to tell us, "What's so annoying about my services is that I have to fill out all this paperwork," and then you turn around and we refer you to them [a different service] and you basically have to fill out the same paperwork, it's just different letterhead. We can see that it's hard, but having them tell us is a different story. 
Her description reveals how efforts to protect clients' identities creates barriers and excludes them from more open, collaborative accessibility, both for client families and for human service professionals across disciplines and organizations. We can also see how enrollment or service initiation processes may be repetitive and duplicative which further excludes client families in subtler ways.

Documentation procedures within and across multidisciplinary human service organizations was described by a majority of participants as exceedingly time consuming and often overwhelming in ways that detracted from direct work with client families. During my participant observations I collected samples of documentation and forms for review according to a constant comparison method of analysis. I was told several times by several different types of professionals the statement, "If it's not documented, it didn't happen." I, too, was familiar with this comment, and I remembered using it while as a supervisor to impress upon junior staff the critical importance of tracking and monitoring service contacts. Client families are often first and/or primarily encountered through forms and documentation; therefore, their legitimacy (or lack thereof) may be contingent upon the plethora of information that is conveyed through agency-to-agency transfer of case documentation through a variety of modes. For example, during participant observations I witnessed collaborations wherein two or more human service professionals from different agencies discussed their knowledge of families using separate, duplicative referral and assessment forms as the primary sources of information. Disparities in the family information (as provided or reported by the family) recorded on the forms led some human service workers to doubt and criticize certain family members' honesty and personal integrity. 
Excluding client families from opportunities to correct or clarify such disparities or chances to contextualize their crisis situations perpetuates and sustains differentiallysubjective power relations between professionals and clients. Ethically, families have these rights, yet, realistically, few human service professionals feel they have available work time to follow up with families in such a profoundly vital and important way. Other workers, often unintentionally, disregard the need to clarify information with client families, especially when knowledge of families is discursively exchanged between authoritative and legitimate sources within professional, often specialized, ranks of expertise.

The professionalism and codes of ethics that are used as standards that guide human service workers are exclusionary cultural characteristics Lorber (1994) refers to as forms of "homosociality" (p. 231). She defines homosociality as a contemporary form of bonding, "In twentieth-century businesses, professions, and politics, trusty and loyalty are built not through kinship ties (which is consider nepotism) but through homosocialitythe bonding of men [sic] of the same race, religion, and social class background." The professional culture of human services co-constructs and perpetuates inclusionary and exclusionary boundaries which are types of categorical power that leads to durable inequality (Tilly 1998). At-risk families are marginalized on the periphery of professional culture which has a tendency towards homosociality. The homosociality of a local social network of human service professionals was described by a law enforcement officer who commented:

Collaborating with the people we usually collaborate with, people who become police officers, social workers, teachers, nurses - we are all about the same people. There's a reason out there that you see a bunch of cops, I bet 90 percent of us are married to teachers or nurses. We're all cut out of 
the same bolt of cloth. Talk to those four groups-cops and social workers, teachers and nurses - we are all, and you can lump firemen in there, too, to make it rounded out, we all had a middle class upbringing, we had a real strong work ethic because our parents were hard workers, we are help oriented, we're service oriented people. That's why I think we're attracted to each other. That's why you see so many cops marrying teachers and nurses, because we're all in that same sort of class we get along pretty well. We identify with each other.

His comment not only supports the homosocial context of a local professional child welfare network, but it further describes how personal and intimate relationships form as a result of such similarities. In this sense, the idea of a community of human service professionals is realized which works to further embed, sustain, and perpetuate the professional culture. The parallel to this idea is offered by a family law attorney who aptly illustrated the cultural distance between a professional discipline or community and the lived realities of the client families they serve. He shared:

I think most legal professionals have probably never lived a lot of the conditions that the litigants have lived. I don't think very many lawyers have direct personal knowledge and understanding of the remarkable, broad-spectrum instability that so many of these families struggle with. A lot of the attorneys involved in family law practice, they're not rich people. Frankly, most of them wish they could get enough good paying clients that they can stop doing court-appointed work, and I'm on that list because that means I've got good paying clients and I don't have to go do GAL [Guardian ad litem] work or court-appointed work, so they're not the wealthiest members of the bar, but the relative privilege they enjoy from having a professional education and a professional degree and a job that's respected, a white collar job, is something that I just think for most people, even if they're well intentioned and good hearted, naturally prevents them from being able to fully identify and empathize with where so many of these families are coming from.

His excerpt, similar to the preceding comment, demonstrates a number of important points. Human service professionals share a culture from which the recipients of their services are often excluded. Their privilege and power is 
relative, but certainly disproportionately greater than client families. Lastly, the nature of human service work often leads professionals to be their own subject of focus which many times displaces and excludes client families from realistically being a predominant priority.

When client families willingly or unwillingly submit themselves as recipients of human services, they unknowingly (but sometimes knowingly) relinquish power over their own decision-making and outcomes. They yield their own power to the subjective will of others who are entrusted to keep their best interests in mind and at heart, and to the influence of expertise, often involuntarily as a result of legislative authority, which is also a pathway for coercive and exclusionary power. Yet, what is deemed to be in the best interest for any family can be very subjective and can look profoundly different from discipline to discipline and from professional to professional, even within the same discipline. Client families experience both professional exclusion and cultural exclusion, and this may be a deeper sense of alienation than oppression. Rather it is a compressive form of alienation and social pressure because it is experienced in deeply meaningful ways from within and from without, both internally and externally. These social expectations are socialized, internalized, and normalized according to Berger (1963) who stated:

The key term used by sociologists... is internalization. What happens in socialization is that the social world is internalized by the child. The same process, though perhaps weaker in quality, occurs every time the adult is initiated into a new social context or a new social group. Society, then, is not only something 'out there,' ... but it is also 'in here,' part of our innermost being. Only an understanding of internalization makes sense of the incredible fact that most external controls work most of the time for most of the people in a society. Society not only controls our movements, but shapes our identity, our thought and our emotions. The structures of society become the structures of our own consciousness. Society does not 
stop at the surface of our skins. Society penetrates us as much as it envelopes us. (p. 121)

At-risk families carry the penetrating, internal weight of not meeting societal standards for "normal" families and the enveloping, external burden of negotiating (often unsuccessfully) a dense network of intersectional, hyper-complex, integrated, multidisciplinary human service systems situated within a broader gendered culture of structural inequalities.

The interplay of relative, subjective, and exclusionary power within human services is a potent and complex mixture of macro and micro-level social dynamics which, once acknowledged, cannot be overlooked. However, in critical ways my own experience of doing this dissertation research paralleled many of the barriers that participants described, especially in terms of relative, subjective, and exclusionary power. A profound limitation of this study was that the nature of academic timelines and budgetary constraints did not allow for the inclusion of family participants who had received human services. Their standpoint, though peripherally represented, continues to be excluded and so, for now, their own voices remain silenced.

Both client families and professionals often suffer in silence. My own experience of doing human services was often uncomfortable, and it was extraordinarily difficult at times to express whatever concerns I had. As difficult as it could be, though, I found hope, motivation, and inspiration through relationships - both with colleagues and with clients. Many of my professional relationships became lasting friendships, and other professional relationships helped to make my dissertation research possible.

My dissertation was an opportunity to voice many essential concerns that those who do human services and those who receive human services regularly encounter. To 
say that human services are merely complex is over simplistic, and to say that human service organizations are merely another form of complex organizations is incomplete. In Chapter Six, Discussion and Conclusions, I layout an alternative explanation, one that potentially has greater explanatory power to describe the dense interconnections of cultural and structural systems interlocked with hierarchies of dominance and power. I also discuss recommendations for confronting barriers and constraints to interagency collaboration, many of which were provided by the study participants, at the end of Chapter Six. 


\section{CHAPTER SIX}

\section{DISCUSSION AND CONCLUSIONS}

The dissertation research was conceptualized to explore dimensions of gender and power within the process of interagency collaboration among human service organizations. The prevailing themes that emerged from the data showed that gender and power are situated ubiquitously throughout organizational settings, including human services, and both social forces profoundly shape the ways in which collaboration either works well or becomes constrained. Candace's story and the many personal experiences shared by multidisciplinary participants in this dissertation further reflected the virtuosity of power in shaping human lives.

\section{Gender, Power, and Collaboration}

My findings aligned with the works of Carol Gilligan (2000) and Sandra Bartky (1990) among others (Murray 1996; Martin 2006; Newman 2007; Noddings 2013) who emphasized the ways in which human services are organizational environments founded within moral philosophies that evolved out of ethics of care. Many professional disciplines within human services have been characterized as female domains or as forms of women's work such as teaching, nursing, child care, and various forms of case management, especially those types that function in a partially clerical role. Conversely, bureaucracy, as an ethic of justice, has been viewed as more masculine in orientation, a cultural tradition aligned with the idea of the workplace as a male domain. My findings indicate that interagency collaboration is situated within this contested space. The more 
bureaucratized human service organizations have become, the more care work has been eclipsed by a masculinized ethic of justice, or bureaucracy.

The value of establishing professional relationships, a gendered process, was a major point of consensus among the participants and was evident during participant observations. The previous survey findings also implicated a number of key factors deemed central to establishing relationships, such as facilitating opportunities to exchange agency or programmatic information and sharing successful outcomes associated with effective collaborations (Moore 2014). The qualitative findings expanded upon the survey findings by situating gender and power within processes of multidisciplinary interagency collaboration including relationship building and maintenance.

Initiating and building relationships within human services may serve many purposes. In terms of crisis work, intra and inter-organizational relationships may help to sustain motivation and persistence in high stress work environments as well as retention of workers. Previous studies have further shown relationship management to be an effective public relations strategy, both in terms of marketing public services to other professionals as well as to clientele with specialized needs (Bruning and Ralstron 2000; Bruning 2002).

Relationship maintenance within human services becomes a more tenuous venture when we consider many of the bureaucratic barriers or constraints to collaboration presented in the findings. Organizational factors such as high stress, high caseloads, competition, and high turnover mean that relationships are harder to develop or sustain. Moreover, much of the work of human services is involuntary and regulated by 
legislative statutes, court orders, and policy mandates; therefore, both professionals and client families are regularly tasked with high-pressure, high-stakes obligations they might not personally choose to do on their own. In these moments in particular, ethics of justice couched within legal-rational modes of bureaucracy may truly overpower ethics of care within crisis work, gendered dynamics that critically shape outcomes for both professionals and for client families.

Establishing relationships takes time. One unfortunate paradox that my study revealed is that the crucial element for enhancing interagency collaboration through establishing relationships (which includes foundational elements such as communication, trust, reciprocity, openness, familiarly, receptiveness, and interest, among others), is time, which is constrained by time itself. Human service workers are inundated with systemic complexities including accounting for the time they spend with families, which limits time for providing direct care to at-risk client families. A critical deficit of available time and energy may chronically and pervasively disrupt the continuity of established relationships.

Another paradox related to time and relationships was illustrated by the emphasis many participants placed on new workers, tenure, and turnover. The longer professionals remain in human services roles the more opportunities they have to build collaborative relationships over time. Yet, bureaucratic constraints were described as primary reasons for resignations, terminations, and other types of job transitions. Increasing rates of turnover and rapidly fluctuating professional roles disrupt opportunities to sustain stable collaborative relationships. Thus, both new and tenured professionals may increasingly lose interest in building relationships or investing valuable and scarce resources, such as 
time, in maintaining collaborations gauged to be potentially unstable. A third paradox my study demonstrated is how collaboration has become a broad scale systemic response to the increasing complexity of human services. As the gendered organizational field, human services has become increasingly bureaucratized and complex, collaboration, too, has transformed into a series of effort-intensive, often complicated, processes. Further, mandates to collaborate may further intensify existing systemic complexities. According to Hood (2014), “...integrated working, in children's services as elsewhere, can be seen as a response to complexity. Indeed, managing complexity underlies most rationales for integration" (p. 28).

Types of power. My findings demonstrated the presence and demonstrable influence of multiple types of power. Drawing upon Weber (1941), Perrow (2014), Foucault (1980), among others (Goldhammer and Shils 1939; Mintz and Schwartz 1985; Handy 1993; Clegg et al. 2006), I found that masculinized, bureaucratic constraints were both outcomes and perpetuating sources of multiple types of power. In addition to relative, subjective, and exclusionary types of power as explicated in Chapter Five, other types of power as reviewed in the previous literature covered in Chapter Two were also present in my study. Among human service organizations hierarchal power was associated with legitimate power and authority, as well as expert power and connection power (French and Raven 1959; Lipkin 2013). Organizations and professionals who were credentialed or were perceived by other professionals and by client families as having expertise exuded and enacted relative, subject, and exclusionary power.

Martin (2010) refers to expert power exerted within hierarchically-structured human services as professional power and states, "there is power in being the 
representative of an agency such as a local authority; you have legal powers and duties...." (p. 60). Although this type of power may be experienced by other professionals and by client families as coercive, expert or professional power is legitimized through interactional and discursive modes of "ruling relations" (Smith 1987; West and Zimmerman 1987).

Gender, power, and systemic complexities. The dissertation findings revealed innumerable ways in which interagency collaboration within human services is merely one of many intricately complex organizational processes at work within a broader institutionalized structure of systemic complexities. Much of my research aligned with previous and current literature; however, few if any previous studies have explored dynamic, intersectional qualities of human service organizations within the context of broad scale systemic collaboration and relative intersectional complexities; thus, this dissertation and my future work addresses this gap in the literature. Previous research has considered the intersectional dimensions of gendered organizations (Kanter 1993; Lorber 1994; Britton 2003; Martin 2006; Acker 2008; Kimmel 2014), and has explored dimensions of power embedded within bureaucratically-structured organizational settings (Weber 1968; Foucault 1977; Tilly 1998; Pfeffer and Salancik 2003; Perrow 2014). These studies lack an interpretation of the many types of power that are situated within and across densely integrated social systems and the vast complement of social structures that compel and collide, as well as condense and constrain social action within pluralities of convergence.

My research offers a closer glimpse at how intersectional systemic complexities are integrated and directly (and indirectly) influence gendered organizational processes, 
primarily interagency collaboration. One potential description that emerged during analysis of the data is a model I refer to as intersectional hyper-complexity. In the next section I will describe foundational considerations of this descriptive model and present a visual interpretation constructed for further explication (see Figure 4 on p. 161 and Table 2 on p. 162). It is important to note that this model is in the process of being explicated further as I continue to analyze the data, and will be a focus of future research as I work towards solidifying theoretical applicability.

\section{Intersectional Hyper-Complexity}

The participants in my study consistently described multiple systemic aspects of human services as complex, but they also characterized these inherent complexities as a normal and natural social state, as "just the way human services are," or "that's how human services work." Participants discussed the systemic complexities of working with at-risk families, of bureaucratic constraints to interagency collaboration, of professional and organizational culture, of the community or region in which they worked, of politics and the economy, and of the spectrum of human service organizations, both in general and specific terms. Their stories, in addition to participant observations, coupled with my own professional career in human services, led me to derive intersectional hyper-complexity as a descriptive model grounded in both data and professional experience.

Intersectional hyper-complexity within the context of gendered human services considers the junctures among a plurality of systemic complexities that coexist within human service disciplines and the broader culture that intersect dynamically, concurrently, and synergistically, and in intersectional ways that compound interpersonal 
experiences of institutional inequality. This means that different human service systems intersect at different times and in different ways contingent upon a client family's individualized needs, as well as the relative accessibility of human services that are locally available at the time of crisis. It also means that who the family is, their social characteristics and personal make up, contributes to their experiences of intersectional complexity.

Human services deviate from traditional modes of work in a number of important ways that directly contribute to intersectional hyper-complexity within the field (Hasenfeld 2010). First, human services are client-centered and family-focused; therefore, there is no one standard problem and no one standard resolution. The potential volatility of real and perceived risk issues requires human service professionals to access and manage an unpredictable range of services, and some of those services will be more effective than others. Indeed, a supportive service that works well for one family may not be effective for the next family or for any other because of the unique, individual responses of families or of individual family members to the service.

Second, human services are provided to humans by humans which leaves a lot of room for interpersonal subjectivity regardless of efforts to standardize professional practices. The individuals who do human services choose their professions for very different, and sometimes complex, personal reasons, and their intentions were voiced in this study as passionately caring. Hood (2014) says,

...complexity in the context of child welfare is hardly impersonal; it is something experienced and managed by families and professionals on a daily basis. It is about perception and interpretation, communication and understanding. In complex social systems, interactions between system entities are the relationships between people. It follows that integrated working is more than about 'tools', protocols and processes; 
fundamentally, it is about what happens in complex cases, in which events unfold as a result of a unique configuration of service users, their families, professionals, managers and other people. (p. 29).

Third, human behavior is unpredictable and potentially volatile in ways that pose risks to individuals' safety; thus, professional autonomy grounded in training and experience in human services is necessary in the face of ubiquitous uncertainty. Fourth, all of the factors just mentioned — widespread subjectivity, autonomy, unpredictability, risk, volatility, and uncertainty, among other factors - sustain an organizational culture of systemic complexities within which intersectional hyper-complexity is experienced as normal.

Institutionalized aspects of intersectionality are embedded within the social structure of each system of complexity and the dynamism of intersectional hypercomplexity is patriarchal in orientation and process. So, while human services have evolved from traditions of nurture and care, or women's work, the imposition of legalrational, bureaucratically-structured business frameworks is decisively a masculine model out of which complex intersectional tensions arise. Intersectional hyper-complexity also considers how interacting systems of complexity ebb and flow and change according to time, timing, and timeframes. For example, there are complex legislative mandates that require specific timeframes, such as Kentucky's adaptation of the Adoption and Safe Families Act of 1997 (GovTrack.us 2016). ASFA guidelines specify that children who have been removed from their homes for safety reasons may not remain in foster care longer than 15 out of the last 22 months of out-of-home-care (OOHC) placement (p. 6). CPS workers are mandated by law and by formal policy to begin concurrent planning (dual service planning for returning home or a relative placement as well as the 
contingency of non-familial adoption) after nine months in OOHC. Many contracted human services have similarly imposed time limitations on their services. Family preservation programs must contact families within 72 hours of accepting a referral, and must sustain eight to ten hours of direct, face-to-face contact with client families in their own homes for a range of four to six weeks (see HOMEBUILDERS ${ }^{\odot}$ Standards (Institute for Family Development 2016). If these rigid timeframes are not met, the program's government contract could be nullified and retracted. Every human service profession within every human service discipline adheres to their own set of strictly regimented policies, practices, and procedures, and imposed timeframes may not sync when services are integrated. Such examples illustrate other dimensions of the intersectional, hypercomplex nature of human service systems.

Intersectional hyper-complexity further reflects potential ways in which the amplification of institutionalized, intersectional power is self-perpetuating, isomorphic and hyper-systemic, internalized repeatedly, and enculturated and reinforced as normal on a multitude of levels - personally, interpersonally, and professionally, as well as organizationally and systemically (DiMaggio and Powell 1983). To add further context for derivation, this description builds upon and attempts to expand the intersectional matrix of domination proposed by scholars such as Kimberle Crenshaw (1993), Patricia Hill Collins (1998) and Leslie McCall (2005), and it heavily relies upon the works of Judith Lorber (1994), Joan Acker (2012), Dorothy Smith (1987), Raewynn Connell (2002), and Michael Kimmel (2014), among others, who have explored gender as a social institution (Bartkey 1991; Martin 2004). It also builds upon the environmental systems theories of Luhmann (1984), Bronfenbrenner (1979), and Bowen's Theory of family 
systems (Brown 1999), as well as organizational complexity theory within the context of protective services (Yasenfeld 2010; Hood 2014). My theoretical model is presented below and was originally conceived and oriented from within the context of interagency collaboration. The shape and logistical features of the model mirror Bronfenbrenner's Ecological Systems Model (1979) and Kolb's Learning Styles Model (Kolb 1981).

The terrain of human services and the social institutions through which they intersect, as well as the myriad social problems and needs that necessitate multidisciplinary human services, are vastly and systemically complex (Hasenfeld 2010; Hood 2014). Emphasis has been increasingly placed on bureaucratic instincts to rationalize uncompromisingly complex social realities, and this is particularly relevant to child welfare. The subsequent effectiveness of workers with families, co-workers, and subordinates appears to be affected by gender, race, credentials, licensure, education, and authority (e.g., courts and law enforcement) and the intersections of these and potentially other statuses such as social class, sexual orientation, and age, among others. Intersectional hyper-complexity is still being developed as a description that may lead to subsequent theory, but, for the purposes of the current study, it is based upon six foundational descriptive considerations:

1. Both family and community systems are multifaceted and dynamically complex. The situations at-risk families, in particular, confront intensify intersectional complexities within family systems and across all other systems they encounter.

2. Numerous multidisciplinary professions make up the organizational environment of human service systems. But, each multidisciplinary 
profession is singularly and dynamically complex and operates within its own professional and organizational culture.

3. At the same time, multidisciplinary human service professions are integrated within and across systemically complex organizational and interpersonal processes, including interagency collaboration.

4. The convergence of dynamically integrated localities of systemic complexities creates a state of intersectional hyper-complexity — complexities that overwhelmingly compound the experience of being involved in human services - that comes to be viewed as normal, natural, just how it is and the way things are.

5. Systemic complexities - much like the broader culture through which they are socially constructed, perpetuated, and maintained — are learned, shared, generationally transmitted both organizationally and professionally, and adapted over time.

6. Organizational localities of systemic complexities are structured and filtered through the broader gendered culture and society, which is by its nature an intersectional and patriarchal experience. The formal and informal social interactions of families, communities, human service organizations, and professionals re-construct and perpetuate categorical intersections of power and domination.

These foundational considerations provide underlying context for understanding, mapping, and conceptualizing the intersecting localities of systemic complexities presented in the descriptive model and descriptive table below: 
Figure 4. Descriptive Model of Intersectional Hyper-Complexity

\section{INTERSECTIONAL HYPER-COMPLEXITY}

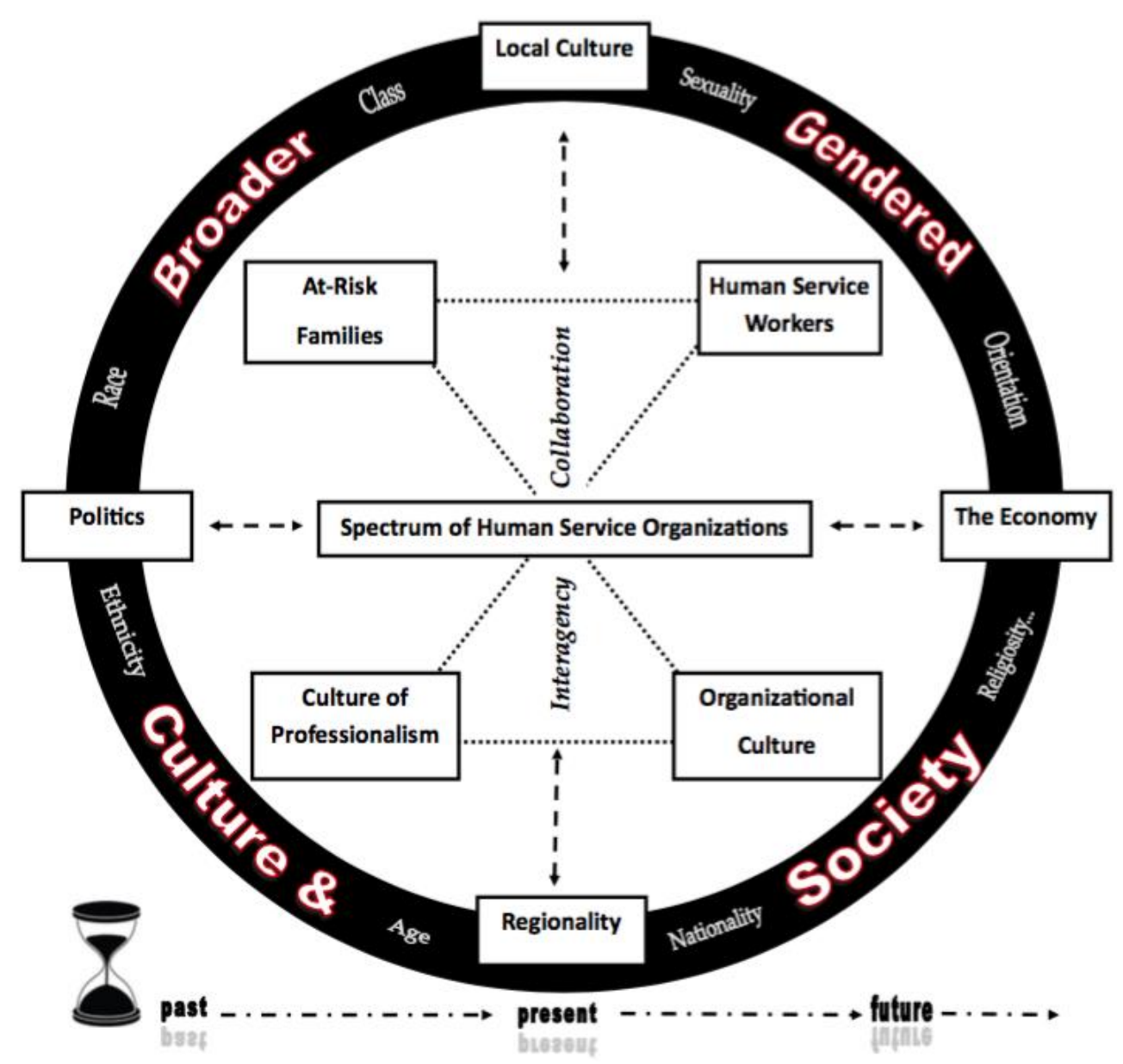

My intentions for the preceding model, subsequent list, and descriptions of localities, or sources, of systemic complexities are twofold. First, the model is a visual representation of the primary institutional systems and human service systems involved in intersectional, hyper-complex multidisciplinary service integration. These dynamically complex systems shaped predominant factors implicated by the participants in my study as bureaucratically imposing and constraining, especially in terms of interagency collaboration. Second, the subsequent table includes a list and descriptions that illustrate 
Localities of

Types of Systemic Complexities

Systemic

Complexities

\begin{tabular}{|c|c|}
\hline $\begin{array}{r}\text { Interagency } \\
\text { Collaboration }\end{array}$ & $\begin{array}{l}\text { - Competition } \\
\text { - Relationship building and maintenance } \\
\text { - Service integration or fragmentation } \\
\text { - Interdependence, resource dependence }\end{array}$ \\
\hline $\begin{array}{r}\text { Spectrum of } \\
\text { Human Service } \\
\text { Organizations }\end{array}$ & $\begin{array}{l}\text { - Multidisciplinary integration } \\
\text { - Surplus or deficiency of services } \\
\text { - Hierarchal power relations - rank, credentials, expertise } \\
\text { - Established entities and new or unfamiliar services } \\
\text { - Funding structure and apparatus - Government, Corporate, Public, Private, For-Profit, Non-Profit, } \\
\text { Volunteer, Faith-Based }\end{array}$ \\
\hline $\begin{array}{r}\text { At-Risk Families } \\
\text { and Children }\end{array}$ & $\begin{array}{l}\text { - Multi-problem, poly-abuse } \\
\text { - Low to moderate to high risk needs } \\
\text { - Crisis and Safety } \\
\text { - Cultural Diversity of Family Forms and Parenting Practices } \\
\text { - Informal Networks and Secondary Supports or the lack thereof }\end{array}$ \\
\hline $\begin{array}{r}\text { Human Service } \\
\text { Workers }\end{array}$ & $\begin{array}{l}\text { - Codes of Ethics, Justice, and Professionalism, Fidelity } \\
\text { - Caring regulated by Bureaucratic Constraints } \\
\text { - Liability and Professional Accountability } \\
\text { - Personal Background, Family and Social Life } \\
\text { - Role Conflict and Role Strain } \\
\text { - Coping and Health, Self-Care and Burn Out }\end{array}$ \\
\hline $\begin{array}{r}\text { Professional } \\
\text { Culture }\end{array}$ & $\begin{array}{l}\text { - Education and Training } \\
\text { - Adopting One or More Sets of Ethical Codes and Guidelines } \\
\text { - Policies, Practices, and Procedures } \\
\text { - Learning the Ranks } \\
\text { - Expertise and Credentials or the Lack Thereof }\end{array}$ \\
\hline $\begin{array}{r}\text { Organizational } \\
\text { Culture }\end{array}$ & $\begin{array}{l}\text { - Stability and Environmental Survival } \\
\text { - Regimentation, Regulatory Bodies of Oversight - Boards, Committees, Accreditation } \\
\text { - Employee Transitions, Turnover, Promotion, Demotion } \\
\text { - Hiring, Training, Socialization and Re-Training } \\
\text { - Niche Specializations, Eligibility, Accessibility, Feasibility } \\
\text { - Budgeting, Contracts, Strategic Planning } \\
\text { - Resource Development and Mobilization } \\
\text { - Isomorphic Trends and Tendencies }\end{array}$ \\
\hline Regionality & $\begin{array}{l}\text { - Urban or Rural } \\
\text { - Family Court or Not, Model Courts or Not } \\
\text { - Accessibility of Services - Transportation, Modes of Communication } \\
\text { - Receptivity, Turf and Terrain } \\
\text { - Orientation to At-Risk Families } \\
\text { - Population Diversity within the Region }\end{array}$ \\
\hline $\begin{array}{r}\text { Local } \\
\text { Culture }\end{array}$ & $\begin{array}{l}\text { - Genealogy and Heritage } \\
\text { - Community Cohesiveness } \\
\text { - Local Jobs and Opportunities } \\
\text { - Social Class, Mobility, and Poverty } \\
\text { - Demographics of the Community } \\
\text { - Geographical Location and Proximity to Urban Areas }\end{array}$ \\
\hline $\begin{array}{r}\text { Economic } \\
\text { Systems }\end{array}$ & $\begin{array}{l}\text { - State and Local Economies } \\
\text { - Funding Cycles } \\
\text { - Accessibility of Public and Private Funding }\end{array}$ \\
\hline Political Systems & $\begin{array}{l}\text { - Election Cycles } \\
\text { - Party Affiliation and Philosophies } \\
\text { - Political Stance on Care, Justice, and Necessity of Human Services } \\
\text { - Earmarks, Lobbying, and Constituencies }\end{array}$ \\
\hline $\begin{array}{r}\text { Broader Gendered } \\
\text { Culture and } \\
\text { Society }\end{array}$ & $\begin{array}{l}\text { - Intersectional Dynamics - Gender, Race, Class, Ethnicity, Nationality, Age, Religiosity and Sexual } \\
\text { - Inientation, among others } \\
\text { - Hegemonic Ideologies } \\
\text { - Gendered Social Structures, Social Action, and Social Interaction }\end{array}$ \\
\hline
\end{tabular}


major types of systemic complexities located within institutional and human service systems. The table provides a richer context for the myriad ways in which an environment of integrated complexities situated within the broader gendered culture and society can become an amplified, yet normalized, experience of intersectional hypercomplexity.

Intersectional hyper-complexity is in need of additional explication and development, yet it has strong explanatory potential for how intersectional dynamics within an ecology of multi-systemic hyper-complexity generates power that is enacted and experienced as relative, subjective, and exclusionary. What remains unclear are more definitive accounts from professionals and client families about when and how they encounter intersectional, multi-systemic complexities within the context of human service systems and how such encounters directly link to enactments of power. My study focused on and emphasized social experiences related to gender and power within organizational processes of interagency collaboration; therefore, more information is needed in order to promote theory development.

Revisiting Candace's story. In Chapter Five, I used Candace's story as a composite case study to demonstrate one client family's potential case progression through multidisciplinary, integrated human service systems including child protective services, family court, law enforcement, foster care, and different types of supportive human service systems. What I understand as an "insider" and what was further reinforced by participants throughout each phase of my study is that intersectional dimensions of human services are overwhelmingly complex and shape dimensions of 
power. As suggested in the proposed description, this intersectional experience is more aptly conceptualized as hyper-complex.

Consider the following "what-ifs" from Candace's story that illustrate the structural junctures and systemic complexities that were not previously revealed and that further support the proposed description of intersectional hyper-complexity. How would Candace's story or our reaction to it have changed if we had learned earlier in the story that domestic violence had been occurring between Tiffany and Bryan the entire time? What would our response had been if we knew that both Bill and Debra had caseloads of 25 or more families with hundreds of children at risk of abuse and neglect similar to what Candace and Drew had experienced?

What if we knew that the judge presiding over their case was gearing up for the next election cycle and had limited time to read and review case notes? Even if she did have available time, what if I revealed that she may not be the same judge who determines if Candace and Drew return home because a new judge who has no history with the family may take her place? How would the story change if we learned that Bill is on the verge of a heart attack because of the stress of doing CPS work, or that Debra may be sanctioned for not having sufficient time to document her hundreds of contacts with clients?

The "what ifs" are endless, but the larger point is a crucial one. Human service systems are constantly in flux, and this is a cultural reality of doing human services work-rampant systemic complexities and repressive stratums of uncertainty couched within hegemonic regimes of normal, everyday cultural practices, practices that evoke relations of power. 


\section{Limitations of the Study}

The dissertation research had four major limitations. First, and most importantly, client families were not formally included in the study. Although services to client families and other forms of social interaction were included in my participant observations, the research scheme and sampling frame excluded family members as current or previous recipients of human services. This is a problematic limitation because an exploration of gender and power within human services is incomplete without including the perspectives of the families and children as recipients and primary stakeholders. Their exclusion also means the reality of their lived experiences of interagency collaboration and other organizational processes is missing from the interpretive analysis of the findings. Families' exclusion from this study mirrors their broader exclusion from many aspects of their own discursive storytelling and decisionmaking, and this is a further imbalance of power.

A second aspect of the study that was both a profound strength and a limitation involved power enacted through my role in the dissertation research. My role was threefold as I was identified as a doctoral candidate, an experienced human service professional, and an external researcher. My career experiences in human services provided an invaluable context for the dissertation research. Having prior working relationships and being known within the geographical area for my professional work in human services gave me opportunities for open access to multidisciplinary professionals, organizational sites, and confidential information that few others may have obtained. Nevertheless, I was also aware of others' reactions at times to my own power, both real and perceived, enacted through professional and academic expertise. I must 
acknowledge, too, the potential for bias, assumptions, and presumptions as an insider exploring a familiar social world. My personal and professional identity, like the identities of my participants and those I observed, is vested in my career experiences, qualifications, and expertise; however, many of the social factors that shape these attributes are steeped in relative, subjective, and exclusionary power. Although I was both mindful and careful, I cannot deny power dynamics are always present at some level, either conscious or unconscious, in professional contexts.

As a feminist researcher, too, interactions with individuals who were subjectively labelled as "participants" or as "subjects" carried the weight (and the privilege) of power. The research relationship is established through building trust and rapport; however, because of the high level of expertise and limited availability of some participants, I was unable to negotiate or establish interpersonal rapport as I would have preferred. The multidisciplinary nature of the study was an overall strength, but, at times, professional rank, differing philosophies, professional distance, and hierarchal authority became sources of limitations.

The inaccessibility of certain human service organizations and lack of broader representation was another limitation of the study. Some organizations such as homeless services, domestic violence services, and other local non-profit agencies were either unavailable to participate in the study or I ran out of time to follow-up further in scheduling interviews. Representation of the majority of participating agencies was on a small-scale, micro-level of social interaction with limited access to the everyday culture of more macro-level organizational processes. The representational context of county, state, and federal policies and legislation similarly needs further explication. 
Other limitations were methodological in orientation. The use of multiple qualitative methods was an effective strategy for my dissertation research; however, each method used had its own unique limitations. Triangulating the methods helped to offset methodological weaknesses. Nevertheless, research-specific limitations were encountered.

The qualitative methods were informed by the use of a quantitative survey, and their use represented an effective strategy for delving more deeply into the previous, very general, and non-specific survey responses. Limitations of the qualitative methodsparticipant observation, focus group interviews, and in-depth interviews-included small sample sizes, especially relative to representatives from each of the organizations that participated in the study, and most of the organizational disciplines in the study were represented by only one or two professional human service workers.

\section{Implications for Future Research}

Human service organizations offer a rich area for critical inquiry. My research presented myriad ways in which the lived experiences of interagency collaboration intersected with gender, race, rank, licensure, and region, among other factors through which power was exercised and carried out within bureaucratized, organizational settings. Gendered intersections and resulting power exerted within caring fields underlie many of the systemic complexities that shape and reinforce bureaucratic barriers to collaboration. What was missing from my findings was a broader intersectional analysis that links gender more directly to other sources of identity and potential experiences of marginalization or inequality such as class, poverty, sexual orientation, and nationality, among others. These connections were tangentially included in my study, but the child 
welfare literature would benefit from a deeper analysis of more specific intersectional dynamics and experiences within human services. Including client families in future research seems like an essential strategy for obtaining such a deeper analysis.

My findings are generalizable within the context of integrated multi-systemic complex human service organizations operating within a rural geographic location. I plan to work towards the development of a theory based on the description I proposed, intersectional hyper-complexity, further; however, gaps in the literature suggest that dimensions of power within organizational processes including interagency collaboration are lacking a deeper interpretation of systemic complexities. My discussion of intersectional hyper-complexity within and across human service systems and systemic processes demonstrates that multidisciplinary systemic complexities have not previously been framed in this way. More exploration is needed because human lives, both professionals and client families, are being systemically overwhelmed at a multitude of levels - personally, professionally, and organizationally. Such immense stressors may have serious, compounding social impacts that affect health, risk, safety, family intactness, and, potentially, abusive injuries to or actual fatalities of children, family members, and human service workers.

Last, and most importantly, avenues of gendered power continue to be pervasive, but indistinct because of the tacit, everyday nature of institutional inequality which is culturally generated and expressed throughout all social practices. The tensions between ethics of care and ethics of justice are built into the bureaucratic business of doing human services. One pivotal consequence that presents an area for further exposition and 
exploration is the commodification of client families as a base unit of "production" within increasingly corporatized models of providing human services.

\section{Recommendations for Enhancing Interagency Collaboration}

Interagency collaboration has been consistently recognized as a best practice strategy for intervention and prevention-based human services that share a primary goal of protecting families and children from victimization (Smith, Witte, and Fricker-Elhai 2006). A number of previous studies have offered recommendations for promoting more effective interagency collaboration (Bardach 1998; Gray 1989; Kagan 1993; Mattessich, Murray-Close, and Monsey 2001; Blue-Banning, Summers, Frankland, Nelson, and Beegle 2004; San Martín-Rodríguez, Beaulieu, D'Amour, and Ferrada-Videla 2005; Sowa 2008).

According to San Martin-Rodriguez et al. (2005), a number of critical elements are essential for promoting successful and effective collaboration. They identified systemic determinants such as embedding a culture of collaboration into a community's human services social system, its culture of professionalism, and its educational or training system. In addition, they recommended building systemic collaborative practices into the organizational structure of human service organizations in ways that allow for increased time, administrative support, team resources, and increased coordination and communication. Interactional determinants were also considered to be critical elements in their study; thus, they recommended devoting attention to incentivizing voluntary aspects of collaboration as a means to promote a greater willingness to collaborate and as a way to foster group cohesion on collaborative teams built upon trust, mutual respect, and a greater desire to communicate regularly. 
Other scholars (Blue-Banning et al. 2004) have focused more on enhancing collaboration between human service professionals and client families. Their work has demonstrated ways to promote a greater sense of equity and involvement for at-risk families and children who reported feeling excluded and disempowered during their involvement with human service organizations. Specific recommendations included a focus on enriching the quality rather than the quantity of collaborative interactions between professionals and client families as well as taking time to clarify ways in which family members can actively partner with designated agency personnel in planning for shared decision-making and outcomes.

The participants in my study offered similar recommendations, but also many additional recommendations for strengthening collaborative care work, especially within the vein of crisis intervention. In the following sub-sections, I provide an overview of their recommendations as well as discuss points of consensus.

Participant recommendations. According to the participants who participated in the dissertation research, the most essential strategy for enhancing interagency collaboration across integrated multidisciplinary human service systems is by finding ways to build and sustain mutually beneficial relationships. Participants specifically qualified working relationships as those in which direct lines of communication had been well established and in which programmatic or organizational information was regularly exchanged and discussed. Many of the participants described being involved in ongoing community collaboration efforts such as local networks of providers who planned quarterly meetings or brown bag luncheon events during which time they would share service information and coordinate community awareness events for the general public. I 
observed that other organizations, such as FPP as the host agency for the study, regularly sent thank you cards and incentives such as free coffee gift cards to acknowledge community partners who had reciprocated mutually-responsive interagency collaboration.

Having a plan for how each participating organization is involved in a collaboration was noted as another essential means for making interagency collaboration work better. Although a specific model of collaboration was not specifically recommended, taking the time to discuss which aspects of collaboration each representative would be responsible for assuming was noted as a beneficial strategy for making collaboration work. A majority of participants discussed the benefits of negotiating memorandums of agreement (MOA) or memorandums of understanding (MOU) in their work with other organizations and programs. Within human services MOAs and MOUs are commonly used "to establish and outline collaborative agreements, including service partnerships" (Huberman and Klaus 2007).

Emphasis was placed on preparing new or transitioning human service workers for the ways in which an organization frames and approaches interagency collaboration which hearkens back to the critically essential elements offered in the San MartinRodriguez et al. (2005) study. Finding ways to embed collaborative structures and cultural practices into the everyday normal operations of human services is particularly essential for new and inexperienced human service professionals. Additional collaborative strategies included having occasional progress meetings and building relationships. In addition to having a plan, some participants mentioned the need for regular conversations or updates about the progress of interagency collaborations, especially if collaborations helped families to achieve positive and successful outcomes. 
One particularly interesting strategy multiple states, including Kentucky, have invested in utilizes technological, multimedia-based 211 information systems as an access point for human services (FCC.gov 2016; United Way 2016). 211 information systems were authorized by the Federal Communications Commission in 2000 and were implemented to increase access to human services and to make it easier for local agencies to provide information for a variety of needs including basic human needs and resources, physical and mental health needs, employment support, aging supports, disability supports, resources for families, children, and youth, and emergency crisis services such as suicide prevention. The participants in my study strongly advocated for this type of critical information source as another potential resource for strengthening interagency collaboration. Once again, though, very few of them were aware that Kentucky already had 211 in many parts of the state and is in the process of expanding 211 statewide. This gap is in large part due to information inundation and other daily broad scale systemic complexities professionals encounter while doing human services, especially intensive crisis care work.

My own experiences of providing human services in the field as well as witnessing multidisciplinary collaboration and listening to the many voices who represent the spectrum of human services in a local context lead me to make stronger recommendations. What became clear in my study is that multidisciplinary human service professionals, regardless of distinction or organizational sphere, experienced many of the same frustrations and concerns about bureaucratic constraints upon their professional roles and their ability to effectively collaborate. Clearer still are the stifling consequences of the widening gap between ethics of care and ethics of justice. Crises 
such as escalating social worker turnover and staffing shortages in Kentucky and in similar states have resulted from unrealistically high caseloads, crippling budget cuts, and at-risk client populations with ever-evolving, multi-problem needs (Yetter 2016).

If child welfare is indeed a priority at the federal, state, and local levels then serious policy considerations are imperative. Human services are an investment in human lives, but also in cultural and societal stability. Although more money may not resolve every concern, increasing state and local funding would support longevity for human service staff by counteracting a primary reason for turnover in most human service jobs. Increasing and maintaining a qualified work force would greatly enhance accessibility to human services as well as stability in organizational processes such as interagency collaboration. I further recommend finding ways to reduce everyday bureaucratic minutiae, especially duplicative forms of reporting and accountability tracking, so that human service workers can focus on delivering more direct forms of care that are so desperately needed.

\section{CONCLUSIONS}

It can be difficult to have critically reflective conversations about the inherent problems of bureaucratic systems because many people feel powerless to change bureaucracy. Yet, bureaucratic processes are social constructions; therefore, people do have power to make both subtle and lasting changes. Change is hard, and relative power overwhelmingly embedded in systemic complexities makes change profoundly more difficult. Acknowledging differential types of power, however, is a pathway to positive, empowering personal, interpersonal, and inter-professional change. Thus, it becomes critical to find ways to balance ethics of care against the imposition of systemic 
bureaucratic constraints, especially within human services. Doing so is an important social policy issue and a question of social justice for a class of people who, as recipients of integrated multidisciplinary human services, experience stigma and other overt as well as subtler forms of prejudice, discrimination, and marginalization.

Continuing to research the many ways in which intersectional hyper-complexity disrupts or impedes care work, and, in particular, interagency collaboration, is also essential for both professionals and client families. Families and children are more than clients. They are partners and the primary constituents in their own collaborative decision making and this is why it is critical to re-orient power relationships within the professional culture of human services. Without the buy-in and investment of client families in their own supported problem solving, lasting change cannot occur and children will continue to experience pervasive trauma and victimization.

Intersectional hyper-complexity is in need of additional explication and development, yet it has emerging potential. The resonating realization that Candace's story conveys as a composite case study is that thousands of families and children in Kentucky and millions of families and children nationwide are voluntary or involuntary recipients of multidisciplinary human services each year. Statistics on that scale mean that systemic complexities within professionalized human services have become exponentially complicated as have families' multi-problem needs for services.

Recent child welfare trends indicate that the pervasiveness and severity of child maltreatment is worsening, especially in Kentucky which has the highest child maltreatment rates in the nation relative to its child population (US Department of Health and Human Services 2014; PCAKY 2016). According to the Administration for Children 
and Families' Child Maltreatment Report for 2014, Kentucky's rates of substantiated child victims steadily increased from 16.6 children per 1,000 in the population in 2010 to 20.6 as of 2014. The rate for first-time child victims similarly increased from 11.6 in 2010 to 14.4 in 2014.

During state fiscal year 2015 (July 1, 2014 - June 30, 2015) approximately 54,177 CPS reports met criteria for investigation or assessment with a total of 73,102 children involved in those reports (PCAKY 2016). Nearly 26,962 children involved in 15,100 of those reports had a legal determination of substantiation or "in need of services." Substance abuse was documented as a risk factor in $57 \%$ of the reports with a finding of substantiation or services needed. Family violence was documented as a risk factor in $39 \%$ of those reports, and health concerns were documented as a risk factor in $37 \%$ reports. Forty-six percent of children in substantiated or services needed reports were age 5 or younger. From 2011 through 2014 there was a steady increase in the number of reports that met CPS acceptance criteria, so, while there was a 7\% decrease in the number of reports from 2014 to 2015, there was an $18 \%$ increase in the number of children in substantiated / services needed reports. Additionally, there has been a $49 \%$ increase in the number of unique children in substantiated or services needed reports from 2011 to 2015.

The seriousness of child maltreatment in Kentucky, throughout the nation, and worldwide means that the data, the challenges, the infrastructure, and the cultural practices of human services need to be reconsidered, re-interpreted, and revised. What my study offers is an opportunity to critically reflect upon everyday interpretations of gendered organizational processes, including interagency collaboration, and consider the 
ways in which the social practices of human services can both empower and constrain the outcomes we so desperately seek - primarily the protection of vulnerable children from initial or recurrent harm. Acknowledging intersectional forms of power, including gendered forms of bureaucratic power, is an important step closer to reconnecting with ethics of care as a pathway to achieving such a critical goal. 


\section{REFERENCES}

Acker, Joan. 1992. "Gendering Organizational Theory." Classics of Organizational Theory, 463-472.

Acker, Joan. 2004. "Gender, Capitalism and Globalization." Critical Sociology, 30(1): $17-41$.

Acker, Joan. 2008. "Helpful Men and Feminist Support: More Than Double Strangeness." Gender, Work \& Organization, 15(3): 288-293.

Acker, Joan. 2012. "Gendered Organizations and Intersectionality: Problems and Possibilities." Equality, Diversity and Inclusion: An International Journal 31(3): 214-224.

Acker, Joan, Kate Barry, and Joke Esseveld. 1983. "Objectivity and Truth: Problems in Doing Feminist Research." Women's Studies International Forum, 6(4). Pergamon.

Acker, Joan, Kate Barry, and Joke Esseveld. 1991. "Objectivity and Truth: Problems in Doing Feminist Research.” Women's Studies International Forum, 6(4), 423-435.

ACT Government. 2016. "Better Services: Human Services Blueprint - Technical Specifications.” Retrieved June 3, 2016. (https://www.betterservices.act.gov.au/ human-services-blueprint/human-services-blueprint-technical-specifications.).

Adler, Patricia A. and Peter Adler. 1994. "Observational Techniques." Handbook of Qualitative Research, 1(3): 377-392. 
Advocates for Youth. 2016. "Creating and Using a Memorandum of Agreement."

Retrieved on May 28, 2016. (http://www.advocatesforyouth.org/ publications/publications-a-z/616-creating-and-using-a-memorandum-ofagreement).

aiim.org. 2015. “AIIM's Life Cycle Collaboration Model.” Retrieved on March 3, 2015. (http://myorgbio.org/aiims-life-cycle-collaboration-model/).

Ajar, Sandra T., and Corina Benjet. 1994. "A Cognitive Perspective on Ethnicity, Race, and Termination of Parental Rights.” Law and Human Behavior, Jun: 249-268.

Allen, Davina. 1997. "The Nursing-Medical Boundary: A Negotiated Order." Sociology of Health and Illness, 19(4): 498-520.

Altman, Julie Cooper. 2008. "Engaging Families in Child Welfare Services: Worker Versus Client Perspectives." Child Welfare, 87(3): 41.

Aries, Phillippe. 1962. Centuries of Childhood: A Social History of Family Life. New York, NY: Vintage Books.

Bardach, Eugene. 1998. Getting Agencies to Work Together: The Practice and Theory of Managerial Craftsmanship. Washington, D.C.: Institution Press.

Barrett, Raymond D. 2013. "Dynamics of Interagency Teams." Military Review 93(2): $53-61$.

Bartky, Sandra Lee. 1990. Femininity and Domination: Studies in the Phenomenology of Oppression. New York, NY: Psychology Press.

Berg, Bruce L. and Howard Lune. 2012. Qualitative Research Methods for the Social Sciences. Boston, MA: Pearson. 
Bergen, Raquel K. 1993. “Interviewing Survivors of Marital Rape: Doing Feminist Research on Sensitive Topics." Pp. 17-211 in Researching Sensitive Topics, Edited by C. M. Renzetti and R. M. Lee. Newbury Park, CA: Sage.

Berger, Peter L. 2011. Invitation to Sociology: A Humanistic Perspective. New York, NY: Anchor.

Bernard, Claudia. 2001. "Child Sexual Assault: Feminist Perspectives." Child \& Family Social Work, 6(3): 280-281.

Berry, Marianne. 1997. The Family at Risk: Issues and Trends in Family Preservation Services. Columbia, SC: University of South Carolina Press.

Biernacki, Patrick, and Dan Waldorf. 1981. "Snowball Sampling: Problems and Techniques of Chain Referral Sampling." Sociological Methods \& Research, 10(2): 141-163.

Blumer, Herbert. 1954. "What is Wrong With Social Theory?" American Sociological Review, 19(1): 3-10.

Blue-Banning, Martha, Jean Ann Summers, H. Corine Frankland, Louise Lord Nelson, and Gwen Beegle. 2004. "Dimensions of Family and Professional Partnerships: Constructive Guidelines for Collaboration." Exceptional Children, 70(2): 167184.

Bowen, Glenn A. 2008. "Naturalistic Inquiry and the Saturation Concept: A Research Note." Qualitative research, 8(1): 137-152.

Britton, Dana M. 2000. "The Epistemology of the Gendered Organization." Gender \& Society, 14(3): 418-434. 
Britton, Dana M. 2003. At Work in the Iron Cage: The Prison as Gendered Organization. NYU Press.

Bronfenbrenner, Urie. 1979. "Contexts of Child Rearing: Problems and Prospects." American Psychologist, 34(10): 844.

Brown, Jenny. 1999. "Bowen Family Systems Theory and Practice: Illustration and Critique." Australian and New Zealand Journal of Family Therapy, 20(2): 94103.

Brown, Jocelyn, Patricia Cohen, Jeffrey G. Johnson, and Suzanne Salzinger. 1998. "A Longitudinal Analysis of Risk Factors for Child Maltreatment: Findings of a 17Year Prospective Study of Officially Recorded and Self-Reported Child Abuse and Neglect." Child Abuse \& Neglect, 22(11): 1065-1078.

Bruning, Stephen D. 2002. "Relationship Building as a Retention Strategy: Linking Relationship Attitudes and Satisfaction Evaluations to Behavioral Outcomes." Public Relations Review, 28(1): 39-48.

Bruning, Stephen D., and Meghan Ralston. 2000. "The Role of Relationships in Public Relations: Examining the Influence of Key Public Member Relational Attitudes on Behavioral Intent." Communication Research Reports, 17(4): 426-435.

Bruns, Eric J., Janet S. Walker, Adam Bernstein, Eric Daleiden, Michael D. Pullmann, and Bruce F. Chorpita. 2014. "Family Voice with Informed Choice: Coordinating Wraparound with Research-Based Treatment for Children and Adolescents." Journal of Clinical Child \& Adolescent Psychology, 43(2): 256-269.

Burns, Barbara J., Sonja K. Schoenwald, John D. Burchard, Leyla Faw, and Alberto B. Santos. 2000. "Comprehensive Community-Based Interventions for Youth with 
Severe Emotional Disorders: Multisystemic Therapy and the Wraparound Process." Journal of Child and Family Studies, 9(3): 283-314.

Butler, Stephen, Leslie Atkinson, Michael Magnatta, and Eric Hood. 1995. "Child Maltreatment: The Collaboration of Child Welfare, Mental Health, and Judicial System." Child Abuse \& Neglect, 119(3): 355-62.

Cabinet for Health and Family Services (CHFS). 2012. “RFP.736.2012.” Frankfort, KY. Cabinet for Health and Family Services (CHFS). 2016. "DCBS Division of Service Regions.” Retrieved on May 17, 2016. (http://chfs.ky.gov/dcbs/serviceregions

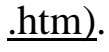

Cabinet for Health and Family Services (CHFS). 2016. "Child Protective Services Case Plan.” Retrieved on June 10, 2016. (http://manuals.sp.chfs.ky.gov/Pages/ index.aspx).

Cabinet for Health and Family Services (CHFS). 2016. "Child Protective Services Prevention Plan." Retrieved on June 10, 2016. (http://manuals.sp. chfs.ky.gov/Pages/ index.aspx).

Cabinet for Health and Family Services (CHFS). 2016. "First Steps Referral Form." Retrieved on June 12, 2016. (http://chfs.ky.gov/dph/firststeps/). Cabinet for Health and Family Services (CHFS). 2016. "HANDS Home Visit Log." Retrieved on June 12, 2016. (http://chfs.ky.gov/dph/mch/ecd/hands.htm). Cabinet for Health and Family Services (CHFS). 2016. "90-Day Concurrent Planning Family Assessment.” Retrieved on June 10, 2016. (http://manuals. sp.chfs.ky.gov/Pages/ index.aspx). 
Cabinet for Health and Family Services (CHFS). 2016. "Universal Referral Form for Services." Retrieved on June 10, 2016. (http://manuals.sp.chfs.ky.gov/Pages/ index.aspx).

Cancian, Francesca. 2000. "Paid Emotional Care: Organizational Forms That Encourage Nurturance." Care Work: Gender, Class, and the Welfare State, 136-148.

Cancian, Francesca M., and Stacey J. Oliker. 2000. Caring and Gender. New York, NY: AltaMira Press.

Charmaz, Kathy. 2003. "Grounded Theory." Qualitative Psychology: A Practical Guide to Research Methods, 81-110.

Charmaz, Kathy. 2014. Constructing Grounded Theory. New York, NY: Sage. Childhelp. 2016. "Child Abuse Statistics \& Facts.” Retrieved on May 15, 2016. (https://www.childhelp.org/child-abuse-statistics/).

Child Welfare Information Gateway. 2016. Retrieved on April 29, 2016 from (https://child welfare.gov/).

Clark, Chris. 2006. "Moral Character in Social Work." British Journal of Social Work, 36(1): 75-89.

Clegg, Stewart R., David Courpasson, and Nelson Phillips. 2006. Power and Organizations. Newbury Park, CA: Pine Forge Press.

Clegg, Stewart R., and Cynthia Hardy. 2012. Handbook of Organization Studies. Thousand Oaks, CA: Sage Publications Ltd.

Clegg, Stewart R., Cynthia Hardy, Thomas B. Lawrence, and Walter R. Nord, eds. 2013. The SAGE Handbook of Organization Studies. Thousand Oaks, CA: Sage. 
Coeling, Harriet V., and James R. Wilcox. 1994. "Steps to Collaboration." Nursing Administration Quarterly, 18(4): 44-55.

Cohen, Marcia. 1998. "Perceptions of Power in Client/Worker Relationships." Families in Society: The Journal of Contemporary Social Services, 79(4): 433-442.

Coleman, Gill, and Ann Rippin. 2000. "Putting Feminist Theory to Work: Collaboration as a Means Towards Organizational Change." Organization, 7(4): 573-587.

Collins, Patricia Hill. 1998. "It's All in the Family: Intersections of Gender, Race, and Nation." Hypatia, 13(3): 62-82.

Connell, R. W. 1987. Gender and Power: Society, the Person, and Sexual Politics. New York, NY: Stanford University Press.

Connell, Robert W. 2002. Gender. Cambridge: Polity.

Coontz, Stephanie. 1992. The Way We Never Were: American Families and the Nostalgia Trap. New York: Basic Books.

Coontz, Stephanie. 1997. The Way We Really Are: Coming to Terms with America's Changing Families. New York: Basic Books.

Coontz, Stephanie. 2011. A Strange Stirring: The Feminine Mystique and American Women at the Dawn of the 1960s. New York, NY: Basic Books.

Corbin, Juliet, and Anselm Strauss. 2014. Basics of Qualitative Research: Techniques and Procedures for Developing Grounded Theory. Thousand Oaks, CA: Sage Publications.

Crenshaw, Kimberle. 1991. "Mapping the Margins: Intersectionality, Identity Politics, and Violence against Women of Color. Stanford Law Review, 43(6): 1241-1299. 
Crossen-Tower, Cynthia. 2002. Understanding Child Abuse and Neglect. Boston, MA: Allyn and Bacon.

Dahl, Robert A. 1957. "The Concept of Power." Behavioral Science, 2(3): 201-215.

Darlington, Y., J. A. Feeney, and, K. Rixon 2005. "Interagency Collaboration Between Child Protection and Mental Health Services: Practices, Attitudes and Barriers." Child Abuse \& Neglect, 29: 1085-1098.

Day, David V., and Robert G. Lord. 1992. "Expertise and Problem Categorization: the Role of Expert Processing in Organizational Sense-Making." Journal of Management Studies, 29(1): 35-47.

Denzin, Norman K. 1994. "The Art and Politics of Interpretation" in Handbook of Qualitative Research. Edited by N. K. Denzin and Y. S. Lincoln. Thousand Oaks, CA: Sage.

Denzin, Norman K., and Yvonna S. Lincoln. 2011. Handbook of Qualitative Research. $4^{\text {th }}$ ed. Thousand Oaks, CA: Sage.

Department for Community Based Services (DCBS). 2008. “Kentucky's Family Preservation Program: Comprehensive Program Evaluation.” Frankfort, KY.

DeWalt, Kathleen M., and Billie R. DeWalt. 2011. Participant Observation: A Guide for Field Workers. $2^{\text {nd }}$ ed. New York, NY: AltaMira Press.

DiMaggio, Paul J. and Walter W. Powell. 1983. “The Iron Cage Revisited: Institutional Isomorphism and Collective Rationality in Organizational Fields." American Sociological Review, 48(2): 147-160.

Doorewaard, Hans, and Birgit Brouns. 2003. "Hegemonic Power Processes in Teambased Work." Applied Psychology, 52(1): 106-119. 
Ely, Robin J., and Debra E. Meyerson. 2000. "Theories of Gender in Organizations: A New Approach to Organizational Analysis and Change." Research in Organizational Behavior, 22: 103-151.

England, Paula. 1992. Comparable Worth: Theories and Evidence. Piscataway, NJ: Transaction Publishers.

Federal Communications Commission (FCC). 2016. "Dial 211 for Essential Community Services.” Retrieved on June 3, 2016. (https://www.fcc.gov/consumers/guides /dial-211-essential-community-services).

Finkelhor, David. 1991. “Child Sexual Abuse.” Pp. 79-94 in Violence in America-A Public Health Approach, Edited by M. L. Rosenberg, and M. A. Fenley. New York: Oxford University Press.

Finkelhor, David. 2009. Children's Exposure to Violence: A Comprehensive National Survey. Darby, PA: DIANE Publishing.

Fitch, Dale. 2009. “A Shared Point of Access to Facilitate Interagency Collaboration.” Administration in Social Work, 33(3): 186-201.

Fonow, Mary Margaret, and Judith A. Cook, eds. 1991. Beyond Methodology: Feminist Scholarship as Lived Research. Bloomington, IN: Indiana University Press.

Fonow, Mary Margaret, and Judith A. Cook. 2005. "Feminist Methodology: New Applications in the Academy and Public Policy." Signs, 30(4): 2211-2236.

Foucault, Michel. 1977. Discipline and Punish: The Birth of the Prison. New York, NY: Vintage.

Foucault, Michel. 1980. Power/Knowledge: Selected Interviews and Other Writings, 1972-1977. New York, NY: Pantheon. 
Fraser, Mark W., and Peter J. Pecora. 1991. Families in Crisis: The Impact of Intensive Family Preservation Services, edited by David A. Haapala. Piscataway, NJ: Transaction Publishers.

Fraser, Mark W., Kristine E. Nelson, and Jeanne C. Rivard.1997. "Effectiveness of Family Preservation Services.” Social Work Research, 21(3):138-53.

French, John R.P., and Bertram Raven. 1959. "The Bases of Social Power." Classics of Organization Theory, 311-320.

Gillham, Bill. 2000. The Research Interview. New York, NY: Continuum.

Gilligan, Carol. 2000. In a Different Voice. Boston, MA: Harvard University Press.

Goldhamer, Herbert, and Edward A. Shils. 1939. "Types of Power and Status." American Journal of Sociology, 171-182.

Gomez, Evangeline. 2012. "The Rise of the Charitable For-Profit Entity.” Forbes. Retrieved on May 2, 2016. (http://www.forbes.com/sites/evangeline gomez/2012/01/13/the-rise-of-the-charitable-for-profit-entity/\#b1bd7d5291cc).

Gottfried, Heidi. 1996. Feminism and Social Change: Bridging Theory and Practice. Chicago, IL: University of Illinois Press.

Graddy, Elizabeth A., and Bin Chen. 2009. "Partner Selection and the Effectiveness of Interorganizational Collaborations." The Collaborative Public Manager: New Ideas for the Twenty-First Century, 53-70.

Gray, Barbara. 1989. Collaborating: Finding Common Ground for Multiparty Problems.

Grosz, Elizabeth. 1990. "Contemporary Theories of Power and Subjectivity." Pp. 59-120 in Feminist Knowledge: Critique and Construct, edited by Sneja Gunew. New York, NY: Routledge. 
Hall, Richard T. 2008. Organizations: Structures, Processes, and Outcomes. Upper Saddle River, NJ: Prentice Hall.

Hamilton, P. 1986. Foreword. In J.B. Bocock Hegemony. London: Tavistock.

Handy, Charles. 1993. "Balancing Corporate Power: A New Federalist Paper." The McKinsey Quarterly, 3: 159-183.

Harbert, Anita, S., Daniel Finnegan, and Nancy Tyler. 1997. "Collaboration." Administration in Social Work, 21: 83-107. doi: 10.1300/J147v21n03_05.

Harding, Sandra G. 2004. The Feminist Standpoint Theory Reader: Intellectual and Political Controversies. New York, NY: Psychology Press.

Hasenfeld, Yeheskel. 2010. Human Services as Complex Organizations. Thousand Oaks, CA: Sage Publications.

Head, George. 2009. "Effective Collaboration: Deep Collaboration as an Essential Element of the Learning Process." The Journal of Educational Enquiry, 4(2).

Held, Virginia. 1993. Feminist Morality: Transforming Culture, Society, and Politics. Chicago, IL: University of Chicago Press.

Heron, Barbara. 2005. "Self-Reflection in Critical Social Work Practice: Subjectivity and the Possibilities of Resistance.” Reflective Practice, 6(3): 341-351, doi: $10.1080 / 14623940500220095$

Hesse-Biber, Sharlene Nagy. 2010. Mixed Methods Research: Merging Theory with Practice. New York, NY: Guilford Press.

Hesse-Biber, Sharlene Nagy and Patricia Leavy. 2006. The practice of qualitative research. Thousand Oaks: Sage. 
Hood, Rick. 2014. "Complexity and Integrated Working in Children's Services." British Journal of Social Work, 44(1): 27-43.

Horner, Gail. 2008. “Child Advocacy Centers: Providing Support to Primary Care Providers." Journal of Pediatric Health Care, 22: 35-39.

Horwath, Jan and Tony Morrison. 2007. "Collaboration, Integration and Change in Children's Services: Critical Issues and Key Ingredients.” Child Abuse \& Neglect, 31(1): 55-69.

"H.R. 867 - 105th Congress: Adoption and Safe Families Act of 1997." www.GovTrack.us. 1997. Retrieved July 3, 2015. (https://www.govtrack.us/ congress/bills/105/hr867).

Hochschild, Arlie. 1983. The Managed Heart. Berkeley, CA: University of California Press.

Huberman, Barbara and Tom Klaus. (2007). "Creating and Using a Memorandum of Agreement." Advocates for Youth. Retrieved on May 15, 2016. (https://www.advocatesforyouth.org/publications/publications-a-z/616-creatingand-usng-a-memorandum-of-agreement).

Hudson, Bob, Brian Hardy, Melanie Henwood, and Gerald Wistow. 1999. "In Pursuit of Inter-Agency Collaboration in the Public Sector: What is the Contribution of Theory and Research?" Public Management an International Journal of Research and Theory, 1(2): 235-260.

Hudson, Bob. 2004. "Analysing Network Partnerships: Benson Re-Visited." Public Management Review, 6(1): 75-94. 
Hugman, Richard. 2014. "Professionalizing Care—A Necessary Irony? Some Implications of the "Ethics of Care" for the Caring Professions and Informal Caring." Pp. 173-1983 in Care Professions and Globalization: Theoretical and Practical Perspectives. New York, NY: Palgrave Macmillan USA.

Hurlburt, Michael, Aarons, Gregory A., Danielle Fettes, Cathleen Willging, Lara Gunderson, \& Mark J. Chaffin. 2014. “Interagency Collaborative Team Model for Capacity Building to Scale-Up Evidence-Based Practice." Children and Youth Services Review, 39:160-168.

Institute for Family Development. 2016. "What is IFPS?” Retrieved February 27, 2016. (http://www.institutefamily.org/).

Institute for Family Development. 2014. “HOMEBUILDERS ${ }^{\odot}$ Standards.” Federal Way, WA.

Johnson, Paul, \& Cahn, Katherine. 2005. "Improving Child Welfare Practice through Improvements in Attorney-Social Worker Relationships.” Child Welfare, 74: 383-394.

Josselson, Ruthellen. 2013. Interviewing for Qualitative Inquiry: A Relational Approach. New York, NY: Guilford Press.

Kagan, Sharon Lynn. 1991, United We Stand: Collaboration for Child Care and Early Education Services. Teachers College Press.

Kagan, Sharon Lynn. 1993. Integrating human services: Understanding the Past to Shape the Future. Yale University Press.

Kania, John, and Mark Kramer. 2011. "Collective Impact." 36-41. 
Kanter, Rosabeth Moss. 1993. Men and Women of the Corporation. New York, NY:Basic Books.

Kanter, Rosabeth Moss. 2008. "Transforming Giants." Harvard Business Review, 86(1): 43-52.

Kempe, C. Henry, Frederic N. Silverman, Brandt F. Steele, William Droegemueller, and Henry K. Silver. 1962. “The Battered-Child Syndrome.” Journal of the American Medical Association, 181(1):17-24. doi:10.1001/jama.1962.03050270019004.

Kennedy, Deseriee A. 2011. "Children, Parents \& the State: The Construction of a New Family Ideology.” Berkeley Journal of Gender, Law \& Justice, 26: 78-138.

Kentucky Revised Statutes. 2016. Section 147A.

Kentucky Revised Statutes. 2016. Section 194A.050.

Kentucky Revised Statutes. 2016. Section 620.

Kentucky Youth Advocates. 2015. 2015 Kentucky KIDS COUNT County Data Book. Louisville, KY.

Kilbourne, Barbara Stanek, Paula England, George Farkas, Kurt Beron, and Dorothea Weir. 1994. "Returns to Skill, Compensating Differentials, and Gender Bias: Effects of Occupational Characteristics on the Wages of White Women and Men." American Journal of Sociology, 689-719.

Kimmel, Michael. 2012. The Gendered Society. $5^{\text {th }}$ ed. Oxford, UK: Oxford University Press.

Kinney, Jill, David Haapala, and Charlotte Booth. 1991. Keeping Families Together: The Homebuilders Model. Piscataway, NJ: Transaction Publishers. 
Kirk, Raymond S., Mimi M. Kim, and Diane P. Griffith. 2005. "Advances in the Reliability and Validity of the North Carolina Family Assessment Scale." Journal of Human Behavior in the Social Environment, 11(3-4): 157-176.

Kleinman, Sherryl. 2007. Feminist Fieldwork Analysis. New York, NY: Sage.

Koehn, Daryl. 1994. The Ground of Professional Ethics. London: Routledge.

Kolb, David A. 1981. "Learning Styles and Disciplinary Differences." The Modern American College, 1: 232-255.

Krug, Etienne G., James A. Mercy, Linda L. Dahlberg, and Anthony B. Zwi. 2002. "The World Report on Violence and Health." The Lancet, 360(9339): 1083-1088.

Langhout, Regina Day, and Elizabeth Thomas. 2010. "Imagining Participatory Action Research in Collaboration with Children: An Introduction." American Journal of Community Psychology, 46: 60-66. doi : 10.1007/s10464-010-9321-1.

Life Instruction Manual. 2015. Bronfenbrenner's Bioecological Model.” Retrieved on May 18, 2015. (http://lifeinstructionmanual.wikispaces.com/Bronfenbrenner $\% 27 \mathrm{~s}+$ Bioecological+Model).

Lipkin, Nicole. 2013. What Keeps Leaders Up at Night: Recognizing and Resolving Your Most Troubling Management Issues. New York, NY: AMACOM.

Lorber, Judith. 1994. Paradoxes of Gender. Boston, MA: Yale University Press. Luhmann, Niklas. 2012. Introduction to Systems Theory. New York, NY: Polity. Lukes, Steven. 1974. Power: A Radical View. New York, NY: Macmillan. Lupton, Carol, Nancy North, and Parves Khan. 2001. "Working Together or Pulling Apart." The Role of Health Professionals in Child Protection. Bristol, UK: The Policy Press. 
March, James G., and Herbert A. Simon. 1958. “Cognitive Limits on Rationality.” Pp. 136-171 in Organizations, New York, NY: Wiley.

March, James G., and Herbert A. Simon. 1993. "Organizations Revisited." Industrial and Corporate Change, 2(1): 299-316.

Martin, Patricia Yancey. 2004. "Gender as Social Institution." Social Forces, 82(4): 1249-1273.

Martin, Patricia Yancey. 2006. "Practising Gender at Work: Further Thoughts on Reflexivity." Gender, Work \& Organization, 13(3): 254-276.

Martin, Patricia Yancey, John R. Reynolds, and Shelley Keith. 2002. "Gender Bias and Feminist Consciousness among Judges and Attorneys: A Standpoint Theory Analysis." Signs, 27(3): 665-701.

Martin, Ruben. 2010. Social Work Assessment. Exeter, UK: Learning Matters Ltd. Mattessich, Paul W., Marta Murray-Close, and Barbara R. Monsey. 2001. Collaboration: What makes It Work. $2^{\text {nd }}$ ed.. St. Paul, MN: Amherst H. Wilder Foundation. Mayhew, Fred. 2012. "Human Service Delivery in a Multi-Tier System: The Subtleties of Collaboration among Partners." Journal of Health and Human Services Administration, 109-135.

McAllister, L., and A. Dudau. 2006. "Gender based Barriers to Effective Collaboration: A Case Study on Children's Safeguard Partnerships." International Journal of Human and Social Sciences, 2: 809-812.

McCall, Leslie. 2005. “The Complexity of Intersectionality.” Signs, 30(3): 1771-1800. Messer, Stanley B., Louis A. Sass, and Robert L. Woolfolk. 1988. Hermeneutics and Psychological Theory. New Brunswick, NJ: Rutgers University Press. 
Meyer, John W., and Brian Rowan. 1977. "Institutionalized Organizations: Formal Structure as Myth and Ceremony." American Journal of Sociology, 340-363. Meyers, Marcia K. 1993. "Organizational Factors in the Integration of Services for Children." The Social Service Review, 547-575.

Mikkelsen, Elisabeth Naima. 2012. "Conflict and Sensemaking Frameworks in Nonprofit Organizations: An Analysis of the Social Meanings of Conflict." Nonprofit and Voluntary Sector Quarterly: 0899764012465674.

Miles, Matthew B., and A. Michael Huberman. 1994. Qualitative Data Analysis: An Expanded Sourcebook. Thousand Oaks, CA: Sage.

Miles, Matthew B., and A. Michael Huberman. 2013. Qualitative Data Analysis: A Methods Sourcebook. Thousand Oaks, CA: Sage.

Miller, JoAnn L., and Dean D. Knudsen. 2007. Family Abuse and Violence: A Social Problems Perspective. Lanham, MD: Rowman AltaMira.

Mintz, Beth A., and Michael Schwartz. 1985. The Power Structure of American Business. Chicago, IL: University of Chicago Press.

Moore, Christa. 2014. "Identifying Gaps in Collaborative Exchange among Human Service Organizations Internship Report.” Unpublished.

Moore, Christa. 2015. “Identifying Motivations, Strengths, and Gaps in Interagency Collaboration." Paper presented at the Association for Applied Clinical Sociology, October 6, 2015, Montgomery, AL.

Moore, Christa. 2016. “Making 'Better' Mothers: Motivations, Strengths, and Gaps in Collaboration.” Panel discussion presented at the Society for Women in Sociology, February 5, 2016, Memphis, TN. 
Moss, Philip, and Chris Tilly. 1996. "'Soft' Skills and Race: An Investigation of Black Men's Employment Problems." Work and Occupations, 23(3): 252-276.

Muhr, Thomas. 1997. ATLAS.ti. Berlin: Scientific Software Development.

Murray Susan B. 1996. "We All Love Charles: Men in Child Care and the Social Construction of Gender". Gender and Society, 10(4): 368-385.

National Children's Alliance. 2016. “Child Advocacy Centers.” Retrieved on January 1, 2016 from (http://www.nationalchildrensalliance.org/index.php?s=24).

National Children's Alliance. 2016. “Child Advocacy Center Model.” Retrieved on January 1, 2016 from (http://www.nationalchildrensalliance.org/cac-model). National Family Preservation Network (NFPN). 2015. “What Is IFPS?” Retrieved on May 3, 2015. (http://nfpn.org/preservation/what-is-ifps).

National Family Preservation Network (NFPN). 2015. "North Carolina Family Assessment Scale for General Services (NCFAS-G).” Retrieved on July 3, 2016. (http://nfpn.org/assessment-tools/ncfas-g-sample).

National Wraparound Implementation Center (NWIC). 2016. "What We Do.” Retrieved on March 28, 2016 (http://www.nwic.org/).

Newman, David M. 2007. Identities and Inequalities: Exploring the Intersections of Race, Class, Gender, and Sexuality. New York, NY: McGraw Hill.

Noddings, Nel. 2013. Caring: A Relational Approach to Ethics and Moral Education. Oakland, CA: University of California Press.

O'Brien, Maeve. 2005. Mothers' Emotional Care Work in Education: The Moral Imperative and Inequalities in Capitals. Dublin, Ireland: University College Dublin. 
Oliver, Christine. 1990. "Determinants of Interorganizational Relationships: Integration and Future Directions." Academy of Management Review, 15(2): 241-265.

Padgett, Deborah K. 1998. Qualitative Methods in Social Work Research. Thousand Oaks, CA: Sage.

Patton, Michael Q. 2015. Qualitative Research and Evaluation Methods: Integrating Theory and Practice. Thousand Oaks, CA: Sage.

Penrod, Janice, Deborah Bray Preston, Richard E. Cain, and Michael T. Starks. 2003. "A Discussion of Chain Referral as a Method of Sampling Hard-to-Reach Populations." Journal of Transcultural Nursing, 14(2): 100-107.

Perrow, Charles. 2002. Organizing America: Wealth, Power, and the Origins of Corporate Capitalism. Princeton, NJ: Princeton University Press. Perrow, Charles. 2014. Complex Organizations: A Critical Essay. Glenview, IL: McGraw Hill.

Pfeffer, Jeffrey, and Gerald R. Salancik. 1978. "Social Control of Organizations" and “The Organizational Environment and How It Is Known.” Pp. 39-91 in The External Control of Organizations: A Resource Dependence Perspective, New York: Standford University Press.

Pfeffer, Jeffrey, and Gerald R. Salancik. 2003. The External Control of Organizations: A Resource Dependence Perspective. New York, NY: Stanford University Press.

Pfeffer, Jeffrey. 1981. Power in Organizations. Vol. 33. Marshfield, MA: Pitman. Pfohl, Stephen J. 1977. "The 'Discovery’ of Child Abuse." Social Problems, 24(3): 310323. 
Prevent Child Abuse Kentucky (PCAKY). 2016. "2015 Child Abuse Data by County.” Retrieved on May 16, 2016. (http://www.pcaky.org/child_abuse_ prevention_month.html).

Reamer, Frederic G. 2001. Tangled Relationships: Managing Boundary Issues in the Human Services. Columbia, SC: Columbia University Press.

Reed, Isaac Ariail. 2011. Interpretation and Social Knowledge: On the Use of Theory in the Human Sciences. Chicago, IL: University of Chicago Press.

Reskin, Barbara. 1993. "Sex Segregation in the Workplace." Annual Review of Sociology, 241-270.

Ridgeway, Cecilia L. 2013. "Why Status Matters for Inequality." American Sociological Review. doi: 0003122413515997.

Ropers-Huilman, Becky. 1999. "Witnessing: Critical inquiry in a Poststructural World." International Journal of Qualitative Studies in Education, 12(1): 21-35.

Rossiter, Amy. 2000. "The Professional is Political: An Interpretation of the Problem of the Past in Solution-Focused Therapy. American Journal of Orthopsychiatry, 70(2): $150-161$

Ruddick, Sara. 2002. “An Appreciation of Love's Labor.” Hypatia, 17(3): 214-224.

Rudman, Laurie A., and Peter Glick. 2012. Social Psychology of Gender: How Power and Intimacy Shape Gender Relations. New York, NY: Guilford Press.

Sáez, Juan, and Mariano Sánchez. 2006. "Trust and Professionalism in Social Professions The Case of Social Education." Current Sociology, 54(4): 595-606.

Sagatun, Inger, and Leonard Edwards. 1995. Child Abuse and the Legal System. Belmont, CA: Wadsworth Publishing. 
Saldaña, Johnny. 2012. The Coding Manual for Qualitative Researchers. Thousand Oaks, CA: Sage.

Saldaña, Johnny. 2014. Thinking Qualitatively: Methods of Mind. Thousand Oaks, CA: Sage.

San Martín-Rodríguez, Leticia, Marie-Dominique Beaulieu, Danielle D'Amour, and Marcela Ferrada-Videla. 2005. "The Determinants of Successful Collaboration: A Review of Theoretical and Empirical Studies." Journal of Interprofessional Care, 19(1): 132-147.

Sandfort, Jodi. 1999. "The Structural Impediments to Human Service Collaboration: Examining Welfare Reform at the Front Lines." Social Service Review, 73(3): 314-339.

Schumacher, Julie A., Amy M. Smith Slep, and Richard E. Heyman. 2001. "Risk Factors for Child Neglect." Aggression and Violent Behavior, 6(2): 231-254.

Shibutani, Tamotsu. 1955. "Reference Groups as Perspectives." American Journal of Sociology, 562-569.

Silverman, David. 2004. Doing Qualitative Research. $2^{\text {nd }}$ ed. London: Sage.

Simmel, Georg. 1971. "Sociability." On Individuality and Social Forms, 127-140.

Singleton, Royce A., and Straits, Bruce C. 2009. Approaches to Social Research. 5th ed. Oxford, UK: Oxford University Press.

Smith, Daniel W., Tricia H. Witte, and Adrienne E. Fricker-Elhai. 2006. "Service Outcomes in Physical and Sexual Abuse Cases: A Comparison of Child Advocacy Center-Based and Standard Services." Child Maltreatment, 11(4): 354360. 
Smith, Dorothy, E. 1987. The Everyday World as Problematic. Boston, MA: Northeastern University Press.

Sowa, Jessica. E. 2008. "Implementing Interagency Collaborations: Exploring Variation in Collaborative Ventures in Human Service Organizations." Administration \& Society, 40: 298-323.

Sprang, Ginny, James J. Clark, and Stephanie Bass. 2005. "Factors that Contribute to Child Maltreatment Severity: A Multi-Method and Multidimensional Investigation." Child Abuse \& Neglect, 29(4): 335-350.

Stark, Evan, and Anne H. Flitcraft. 1988. "Women and Children at Risk: A Feminist Perspective on Child Abuse." International Journal of Health Services, 18(1): 97-118.

Staudt, Marlys, and Brett Drake. 2002a. "Intensive Family Preservation Services: Where's the Crisis?" Children and Youth Services Review, 24.9: 777-795.

Staudt, Marlys, and Brett Drake. 2002b. "Research on Services to Preserve Maltreating Families." Children and Youth Services Review, 24.9: 645-652.

Straus, Murray Murray Arnold, Richard J. Gelles, and Suzanne K. Steinmetz, eds. 2006. Behind Closed Doors: Violence in the American family (1980). Piscataway, NJ: Transaction Publishers.

Symington, Alison. 2004. "Intersectionality: A Tool for Gender and Economic Justice." Women's Rights and Economic Change, 9: 1-8.

Tilly, Charles. 1998. Durable Inequalities. Thousand Oaks, CA: University of California Press. 
Thomas, Eric J., J. Bryan Sexton, and Robert L. Helmreich. 2003. "Discrepant Attitudes About Teamwork among Critical Care Nurses and Physicians." Critical Care Medicine, 31(3): 956-959.

US Department of Health and Human Services. 2013. "The Child Abuse Prevention and Treatment Act (CAPTA) 2010." Retrieved on March 5, 2016. (http://www.acf. hhs.gov/programs/cb/resource/capta2010).

US Department of Health and Human Services. 2014. "Child Maltreatment 2013." Retrieved on February 22, 2016. (http://www.acf.hhs.gov/programs/cb/ resource/child-maltreatment-2014).

US Department of Health and Human Services. 2016. "NCANDS." Retrieved on February 22, 2016. (http://www.acf.hhs.gov/programs/cb/research-datatechnology/reporting-systems/ncands).

United Way of Southern Kentucky. 2016. “Need Help?” Retrieved on June 3, 2016. (http://www.uwsk.org/2-1-1).

VanDenBerg, John E., and E. Mary Grealish. 1996. "Individualized Services and Supports through the Wraparound Process: Philosophy and Procedures." Journal of Child and Family Studies, 5(1): 7-21.

Wallace, Harvey. 2002. Family Violence: Legal, Medical, and Social Perspectives. 2nd ed. New York, NY: Routledge.

Walsh, Wendy A., Theodore P. Cross, Lisa M. Jones, Monique Simone, and David J. Kolko. 2007. "Which Sexual Abuse Victims Receive a Forensic Medical Examination?: The Impact of Children's Advocacy Centers." Child Abuse \& Neglect, 31(10): 1053-1068. 
Warmington, Paul, Harry Daniels, Anne Edwards, Steve Brown, Jane Leadbetter, Deirdre Martin, and David Middleton. 2004. Interagency Collaboration: A Review of the Literature. Learning in and for Interagency Working Project. University of Bath.

Weber, Max. 1941. The" Objectivity" of Sociological and Socio-political Knowledge.

Weber, Max. 1947. "The Theory of Economic and Social Organization." Trans. AM

Henderson and Talcott Parsons. New York: Oxford University Press.

Weber, Max. 1968. On Charisma and Institution Building. Chicago, IL: University of Chicago Press.

Weber, Max. 1978. “Bureaucracy.” Pp. 956-1005 in Economy and Society, edited by R. Guenther and C. Wittich. Berkeley, CA: University of California Press.

Weick, Karl E., Kathleen M. Sutcliffe, and David Obstfeld. 2005. "Organizing and the Process of Sensemaking.” Organization Science, 16(4): 409-421.

Weinberg, Merlinda, and Carolyn Campbell. 2014. "From Codes to Contextual Collaborations: Shifting the Thinking about Ethics in Social Work." Journal of Progressive Human Services, 25(1): 37-49.

Wertz, Frederick J., Kathy Charmaz, Linda M. McMullen, Ruthellen Josselson, Rosemarie Anderson, and Emalinda McSpadden. 2011. Five Ways of Doing Qualitative Analysis: Phenomenological Psychology, Grounded Theory, Discourse Analysis, Narrative Research, and Intuitive Inquiry. New York, NY: Guilford.

West, Candace, and Don H. Zimmerman. 1987. "Doing Gender." Gender \& Society, 1(2): 125-151. 
Wharton, Amy S. 1993. "The Affective Consequences of Service Work Managing Emotions on the Job." Work and Occupations, 20(2): 205-232.

Whittaker, James K. 1990. Reaching High-Risk Families: Intensive Family Preservation in Human Services. New York, NY: A. Gruyter.

Winton, Mark A., and Barbara A. Mara. 2000. Child Abuse and Neglect: Multidisciplinary Approaches, 1st Edition. New York, NY: Pearson.

Wood, Donna J. \& Gray, Barbara. 1991. "Toward a Comprehensive Theory of Collaboration.” Journal of Applied Behavioral Science, 27, 139-162.

Wood, Lisa Anne, Monika Büscher, and Leonardo Ramirez. 2012. Response to Emergence in Emergency Response. Mobilities Lab. Lancaster University, UK.

Yankeelov, Pamela A., Anita P. Barbee, Dana Sullivan, and Becky F. Antle. 2009. "Individual and Organizational Factors in Job Retention in Kentucky's Child Welfare Agency." Children and Youth Services Review, 31(5): 547-554.

Yetter, Deborah. 2016. “Social Worker Crisis Looms in Louisville.” Courier Journal. Retrieved on August 6, 2016. (http://www.courier-journal.com/story/news/ politics/2016/08/05/social-worker-crisis-looms-louisville/87994122/). 


\title{
APPENDIX A
}

\section{IRB-APPROVED INFORMED CONSENT DOCUMENT}

\author{
Subject Informed Consent Document \\ Identifying Gaps in Collaborative Exchange among Human Service Organizations \\ Investigator(s) name \& address: \\ Patricia Gagne, PhD Christa Moore, PhD Candidate \\ Professor \\ Doctoral Student \\ Department of Sociology \\ 103 Lutz Hall \\ University of Louisville \\ Department of Sociology \\ 103 Lutz Hall \\ Louisville, KY 40292 \\ University of Louisville \\ 502-852-8014 (office) \\ 502-767-6930 (cell) \\ Patricia.gagne@louisville.edu \\ Louisville, KY 40292 \\ 270-745-4102 (office) \\ 270-438-8495 (cell) \\ Christa.Moore@wku.edu
}

UofL Institutional Review Boards IRB NUMBER: 15.0616

IRB APPROVAL DATE: 07/17/2015

IRB EXPIRATION DATE: 07/16/2016

Site(s) where study is to be conducted:

The study will take place at a location chosen by research subjects.

Phone number for subjects to call for questions:

502-767-6930

Introduction and background Information:

You are invited to participate in a research study. The study is being conducted by Christa Moore, PhD Candidate and Dr. Patricia Gagne, PhD. The study is sponsored by the University of Louisville, Department of Sociology. The study will take place in the United States at locations chosen by research subjects. Approximately 25 subjects will be invited to participate.

\section{Purpose:}

The purpose of this study is to foster understanding of the experiences of human service professionals before, during, and after participation in interagency collaboration and the ways that gaps in collaborative exchange relative to organizational power dynamics during team decision-making affect that experience.

\section{Procedures:}

In this study, you will be asked to participate in a focus group interview or an in-depth interview or both and to answer questions about your experiences before, during, and (if appropriate) after participating in interagency collaboration as part of the professional work you do within the context of human services. You may refuse to answer any questions that make you uncomfortable. The study will also involve participant observation of family preservation activities, documentation, and clinical records. You may refuse to be observed if it makes you uncomfortable. You may ask for clarification of the study procedures at any time during the study. 


\section{Potential Risks:}

There are no foreseeable risks, although there may be unforeseen risks.

\section{Benefits:}

The possible benefits of this study include a better understanding of the power dynamics involved in interagency collaboration among human service organizations that specifically focus on assisting at-risk families and children. The information collected may not benefit you directly. The information learned in this study may be helpful to others.

\section{Confidentiality:}

Total privacy cannot be guaranteed. Your privacy will be protected to the extent permitted by law. If the results from this study are published, your name will not be made public. While unlikely, the following may look at the study records:

The University of Louisville Institutional review Board, Human Subject Protection Program Interviews will be coded using a number sequence. Information about participants will be obtained from participants directly. Voice recorded interviews will be in physical possession of the researchers at all times. Following transcription, recorded interviews will be destroyed. Transcribed interviews will be protected on a password-protected computer.

\section{Voluntary Participation:}

Taking part in this study is voluntary. You may choose not to take part at all. If you decide to be in this study or if you stop taking part at any time, you will not lose any benefits for which you may qualify. You will be told about any changes that may affect your decision to continue in the study.

\section{Research Subject's Rights, Questions, Concerns, and Complaints:}

If you have any concerns or complaints about the study or the study staff, you have three options.

1) You may contact the principal investigator, Patricia Gagne, $\mathrm{PhD}$ at 502-852-8014 (office) or 502-767-6930 (personal cell).

2) If you have any questions about your rights as a study subject, questions, concerns or complaints, you may call the Human Subject Protection Program Office (HSPPO) (502) 852- 5188. You may discuss any question about your rights as a subject, in secret, with a member of the Institutional Review Board (IRB) or the HSPPO staff. The IRB is an independent committee composed of members of University community, staff of the institutions, as well as lay members of community not connected with these institutions. The IRB has reviewed this study.

3) If you want to speak to a person outside the University, you may call 1-877-852-1167. You will be given the chance to talk about any questions, concerns or complaints in secret. This is a 24 hour hot line answered by people who do not work at University of Louisville. 
This paper tells you what will happen during the study if you chose to take part. Your signature means that this study has been discussed with you, that your questions have been answered, and that you will take part in the study. This informed consent document is not a contract. You are not giving up any legal rights by signing this informed consent document. You will be given a signed copy of this paper to keep for your records.

Signature of Subject/Legal Representative

Signature of Investigator
Date Signed

Date Signed

\section{List of Investigators}

Christa Moore, $\mathrm{PhD}$ Candidate

Patricia Gagne, PhD

\section{Phone Numbers}

270-745-4102 (office)

270-438-8495 (personal cell phone)

502-852-8014 (office)

502-767-6930 (personal cell phone) 


\section{APPENDIX B}

\section{BLANK HOST AGENCY AGREEMENT}

\section{University of Louisville \\ Department of Sociology \\ Dissertation Research Memorandum of Agreement}

Host Agency: [Name of Organization], [County Name], Bowling Green, KY

Doctoral Researcher: Christa J. Moore, MA, Ph.D. Candidate, Applied Sociology Doctoral Program, Department of Sociology, University of Louisville, Louisville, KY

Dissertation Chair: Dr. Patricia Gagné, Department of Sociology, University of Louisville

Dissertation Committee Members: Dr. Dave Roelfs (Department of Sociology, University of Louisville); Dr. Robert Carini (Department of Sociology, University of Louisville); Dr. Gul Marshall(Department of Sociology, University of Louisville); Dr. Kristina Zierold (Department of Public Health, University of Louisville); Dr. Corrie Rice (University Training Consortium, Eastern Kentucky University)

Agency Supervisor(s): [Name of Administrator and Position Title]

\section{Terms of Dissertation Research:}

1) Beginning in May 2015, Christa will learn about the collaborative services and materials offered by the host agency's [Name of Program];

2) she will be authorized to describe and analyze the collaborative services offered by [Name of Program(s)], as well as her experiences working with the organization;

4) she will administer an anonymous survey measuring the general types, strengths, and gaps of collaborative partnerships to [Name of Program] staff and local collaborative partner agencies;

5) she will coordinate and facilitate confidential qualitative interviews measuring the general types, strengths, and gaps of collaborative partnerships with [Name of Program] staff and local collaborative partner agency representatives; and,

3) she will provide a copy of her completed research to [Name of Program].

During and after the time of her dissertation research, Christa Moore will maintain the right to publish articles on the descriptive service data, the collaborative services, and materials offered by [Name of Program], the survey, and the interviews. At all times the identity of the [Name of Program], [Name of Program's] staff, [Name of Program's] partners, and [Name of Program's] clients and the location of the agency and population served will remain undisclosed in publications. Christa Moore agrees to disguise the name of the organization and use 
pseudonyms for all employees, volunteers, interns, and community partners associated with [Name of Program] and that she will provide confidentiality to the organization and its clients.

[Name of Administrator] as an authorized representative of the host agency agrees to the following:

1) $s$ /he will provide Christa Moore with access to approved client records, service data and reports, and related materials that occur during the time of her study, allowing her to observe regularly scheduled activities of the [Name of Program], and that s/he will provide her with free access to films, visual aids, handouts, brochures, and any and all other materials used by [Name of Program] in its training and service efforts;

2) s/he will coordinate a schedule with Christa that allows for on-site observation time and for off-site time to coordinate and administer surveys, attend training sessions, or complete any and all other aspects of data gathering required for the dissertation in affiliation with [Name of Program]. Use of agency materials (occasional copying, printing, work space, computer use, and/or other material needs associated with the study) may be negotiated PRN. Christa will otherwise pay for the cost of the research through her own means or through research funding if available/awarded;

3) s/he will allow Christa to use the host agency's name and organizational information to apply for research funding; and,

4) the Dissertation Committee will have final say on when the Doctoral Dissertation has been completed by Christa Moore (anticipated completion by May 2016).

[Name of Administrator and Position Title]

Ms. Christa Moore, M.A., Ph.D(c)

Dr. Patricia Gagné, Dissertation Chair
Date Signed

Date Signed

Date Signed 


\section{APPENDIX C}

\section{INTERAGENCY COLLABORATION FOCUS GROUP INTERVIEW FORMAT AND QUESTIONNAIRE}

\section{WELCOME AND INTRODUCTIONS}

\section{EXPLANATION OF FOCUS GROUP PROCESS AND “GROUND RULES”}

\section{QUESTIONS}

1. Tell me about your experiences with collaboration [Potential prompts: Do you like it? Prefer not to collaborate? Does it help your work? Does it make it more difficult?] [Ask for examples.]

2. Are there some agencies that are easier to collaborate with than others? [Who are they? What about the agency makes it easier? What about the agency makes it harder?] [Ask for examples.]

3. Tell me about your collaborations with [DCBS, law enforcement, judges, attorneys, therapists, other advocates? What other agencies would you include on a list of collaborative partners? Were collaborative decisions made as a team? Which agencies impacted decision-making the most?]

4. How would you rank the agencies listed in order of most decision-making influence to least decision-making influence? Describe the factors that shaped where you ranked [number $1,2,3 \ldots \mathrm{x}$ ].

5. Has your work been more successful as a result of collaboration? [What shaped the success? If not, what would you have changed about the collaboration?]

6. What other information about interagency collaboration would be important for me to know?

IV. WRAP UP AND QUESTIONS 


\section{APPENDIX D.}

\section{INTERAGENCY COLLABORATION IN-DEPTH INTERVIEW QUESTIONNAIRE}

1. Tell me about your work. [What is your role within the organization? How often do you participate in collaboration as part of your work? What does collaboration mean to you?]

2. Have you been involved in recent collaborations with other agencies? Tell me about your experiences with collaboration. [Do you like collaborating? Prefer not to collaborate? Does it help your work? Does it make it more difficult?]

3. Tell me about your collaborations with [DCBS, law enforcement, judges, attorneys, therapists, other family/child advocates? What other agencies would you include on a list of collaborative partners? Were collaborative decisions made as a team? Which agencies impacted decision-making the most?]

4. How would you rank the agencies you listed in order of most decision-making influence to least decision-making influence? [Describe why you ranked [agency number $1,2,3 \ldots \mathrm{x}]$ where you placed it on the list.]

5. What does having more decision-making influence mean for collaborative teams/work groups? Can you describe a case or a situation wherein you experienced different degrees or types of agency influence on an important decision?]

6. Do any of the agencies on your list refer to your program? [Do you refer families them? Which ones-Agency $[1,2,3 \ldots x$ ?] How has receiving or making a referral source shaped your experience of collaboration?]

7. Describe some specific ways that you have collaborated with other agencies.

8. What factors do you think most affected past collaborations? 
9. How did you know if collaboration was working well?

10. In your experience, what other factors has influenced interagency collaboration? [What about gender? Race? Nationality? Faith-based services? Funding? Competition?]

11. What are your ideas for how collaboration with other agencies could work better?

12. What other information do you feel is important for me to know about your experiences with interagency collaboration? Any questions? 


\section{Appendix E}

\section{Human Services Acronym Guide}

The following list of acronyms is inclusive of the human service organizations, systems, and multidisciplinary professions presented within the scope of my dissertation research. The list is not intended to be nor is it exhaustive of the innumerable terms, concepts, argot, and other discursive aspects of care work.

The terms in this column refer to specific organizations, programs, legislation, or concepts List of Acronyms presented in the dissertation:

Adoption and Safe Families Act of 1997

Barren River Area Development District

Cabinet for Health and Family Services

Child Advocacy Center(s)

Child Protective Services

Department of Community Based Services

Department of Juvenile Justice

Division of Protection and Permanency

Family Preservation Program(s)

Family Resource and Youth Services Center(s)

Growing Up Safe Meeting

Guardian ad litem

Institutional Review Board

Interagency Collaboration Survey

Kentucky Office of Employment and Training

Kentucky Revised Statutes

Multidisciplinary Team(s)

Out-of-Home Care Placement

Potential Removals

Society for the Prevention of Cruelty to Animals

Society for the Prevention of Cruelty to Children

The Workers Information SysTem

\begin{tabular}{|c|}
\hline ASFA \\
\hline BRADD \\
\hline CHFS \\
\hline CAC(s) \\
\hline CPS \\
\hline DCBS \\
\hline DJJ \\
\hline DPP \\
\hline FPP(s) \\
\hline FRYSC(s) \\
\hline GUS Meeting \\
\hline GAL \\
\hline IRB \\
\hline ICS \\
\hline OET \\
\hline KRS \\
\hline MDT(s) \\
\hline OOHC \\
\hline PRs \\
\hline SPCA \\
\hline TWIST \\
\hline
\end{tabular}




\section{CURRICULUM VITAE}

\section{CHRISTA JANE MOORE}

203 Brookshore Avenue

Bowling Green, KY 42101
Mobile:(270) 438-8495

Email: ChristaJ.Moore@gmail.com

\section{RESEARCH AGENDA}

- Interagency collaboration within gendered institutions, enhancing community partnerships, power in team decision-making among human service organizations, organizational feminism

- Organizational responses to family violence, child maltreatment, and abuse prevention

- Ideological constructions of family and related intersections of gender and inequality

- Mixed methods, feminist qualitative methodologies, standpoint methodologies

EDUCATION

$\mathrm{PhD}$, Applied Sociology: Concentration Areas-Gender and Family, Organizations, Mixed Methods, University of Louisville, August 2010 - August 2016

Graduate Certificate, Gender and Women's Studies: Western Kentucky University, August 2012 - May 2016.

MA, Sociology: Western Kentucky University, August 2000 - May 2005.

BA, Psychology: Second Major-Sociology, Minor-Criminology, Honors Program Graduate. Western Kentucky University, August 1993 - December 1998.

\section{COURSES TAUGHT}

Introductory Sociology (On-Site, Online, Dual Credit)

Contemporary Social Problems

Popular Culture

Cultural Diversity (Online)

Interaction, Self, and Society

Introduction to Gender and Women's Studies (Online)

\section{CURRENT EMPLOYMENT}

Full-Time Instructor: Western Kentucky University, Department of Sociology, August 2014 - Present

Part-Time Adjunct: Southcentral Kentucky Community \& Technical College, General Education Division, January 2011 - Present 


\section{PREVIOUS WORK HISTORY}

University of Louisville, Kent School of Social Work, December 2008-August 2014

Data Analyst/Technical Assistant, Positions contracted to the Cabinet for Health and Family Services/Department of Community Based Services/Division of Protection and Permanency/Prevention Branch, Positions located in Frankfort, KY

Western Kentucky University, Sociology Department, 2003, August 2012-May 2014 Adjunct Instructor, Position located at the WKU Glasgow Regional Campus, Glasgow, KY

Community Action of Southern Kentucky, Inc., Family Preservation Department, June 2000- December 2008; Director, 2002-2008, Crisis Intervention Specialist, 2000-2002, Positions located in Bowling Green, KY

Rivendell Psychiatric Hospital, May 2000-September 2000

Mental Health Associate/PRN, Position located in Bowling Green, KY

River Valley Behavioral Health Psychiatric Hospital, February 1999- May 2000

Family Resource Coordinator, Position located in Owensboro, KY

Green River Correctional Complex, June 1998

Case Management Intern, Criminology Internship, Position located in Central City, KY

\section{GRANTS AWARDED}

Family Preservation and Reunification Services, Cabinet for Health and Family Services (written for the Family Preservation Department, Community Action of Southern Kentucky, 2002, 2003, 2004, 2005, and 2007).

Community Collaboration for Children, Cabinet for Health and Family Services (written for the Family Preservation Department, Community Action of Southern Kentucky, 2005 and 2007).

Supervised Visitation Services, Cabinet for Health and Family Services (written for the Family Preservation Department, Community Action of Southern Kentucky, 2005).

Temporary Assistance for Needy Families (TANF) Family Preservation Services, Cabinet for Health and Family Services (written for the Family Preservation Department, Community Action of Southern Kentucky, 2007).

Non-Custodial Parent Access and Visitation Services, Cabinet for Health and Family Services (written for the Family Preservation Department, Community Action of Southern Kentucky, 2007).

Community-Based Child Abuse Prevention, Administration of Children, Youth, and Families (contributed written sections, full review, and edits for the Community 
Collaboration for Children Program, Cabinet for Health and Family Services, Division of Protection and Permanency, Prevention Branch 2009, 2010, 2011, 2012, 2013, 2014).

College of Arts and Sciences Research \& Creative Activities Grant, University of Louisville, 2016

\section{PROFESSIONALPRESENTATIONS}

"Engaging Fathers and Positive Male Role Models in the Lives of Children." Community Wide Workshops, Community Collaboration for Children Project, Bowling Green and Scottsville, Kentucky, August, September, and October 2004.

"Prevention and Treatment of Head Lice in Head Start and In-Home Settings." Head Start Pre-Service Training, Community Action of Southern Kentucky, August 2005.

"Domestic Violence: Indicators and Intervention Techniques." Head Start Pre-Service Training, Community Action of Southern Kentucky, Scottsville, Kentucky. August 2006.

"Non-Violent Crisis Intervention." Transportation Department Annual Training, Community Action of Southern Kentucky, Bowling Green, Kentucky. January 2007.

"Indicators of Child Abuse and Neglect and Mandatory Reporting." Head Start Pre-Service Training, Community Action of Southern Kentucky, Cave City, Kentucky. August 2007.

"Multimedia Advertising: Using Entertainment Media as a Prevention Outreach Tool." Community Collaboration for Children Statewide Meeting, Elizabethtown, Kentucky. September 2007.

"Using Family Team Meetings as a Prevention Tool." Community Partnerships for Protecting Children Quarterly Meeting, Franklin, Kentucky. October 2007.

"Family Preservation and Reunification Services." Racial Disproportionality in Foster Care Ad Hoc Committee, Bowling Green, Kentucky. February 2008.

"Data Protocols and Assessment Training for Community Collaboration for Children Providers." Berea College, Berea, Kentucky. October 2010.

"Child Abuse and Maltreatment." University of Louisville, Louisville, Kentucky. April 2011.

"North Carolina Family Assessment Scale for Generalized Services Training for Community Collaboration for Children." Co-Training at Rough River Lake State Park, Falls of Rough, Kentucky. September 2011.

"HOPE VI: Public Policy Analysis and Housing Options for Low-Income Families." University of Louisville, Louisville, Kentucky. October 2011. 
"North Carolina Family Assessment Scale for Generalized Services Training for Community Collaboration for Children." Co-Training at Best Western, Frankfort, Kentucky. November 2012.

"North Carolina Family Assessment Scale for Generalized Services Training for Community Collaboration for Children." Co-Training at Shelby County Cooperative Extension Office, Shelbyville, Kentucky. February 2013.

"Children and Mortality: A Meta-Analysis." Presented at the Population Association of America Annual Meeting, Boston, Massachusetts, May 2014.

"Identifying Gaps in Collaborative Exchange among Human Service Organizations." Presented at the Association for Applied and Clinical Sociology Annual Conference, Montgomery, Alabama, October 9, 2015

"Identifying Barriers and Realistic Strategies for Managing Stress." Presented at the Suzanne Vitale Special Needs Summit, Bowling Green, Kentucky, October 16, 2015

"Assessment Strategies for In-Home Based Services.” Presented at Uspiritus, Bowling Green, Kentucky, November 12, 2015

"Do Community Programs Make 'Better' Mothers: Identifying Motivations, Strengths, and Gaps in Interagency Collaboration among Human Service Organizations." Presented at the Sociologists for Women in Society Winter Meeting, Memphis, Tennessee, February 6, 2016.

\section{PAPERS IN PROGRESS}

"J-Curve? A Meta-Analysis and Meta-Regression of Parity and Parental Mortality." Robin S. Högnäs, David Roelfs, Christa J. Moore, and Eran Shor. Under Review, Population Research and Policy Review, March 2016.

"Motivations, Strengths, and Gaps in Collaborative Exchange in Human Services." In Progress.

"Contributions of Reference Agents to Recovery Maintenance: A Social World Analysis of Narcotics Anonymous Affiliation.” In Progress.

\section{MEMBERSHIPS}

American Sociological Association Child Welfare League of America

Golden Key Society

Population Association of America

Association for Applied and Clinical Sociology

Sociologists for Women in Society 
- WKU ALIVE Center for Community Partnerships Vision Multi-Agency Council Member

- Barren River Area Community Collaboration for Children (CCC) Regional Network Member

- Family Preservation Program Volunteer 